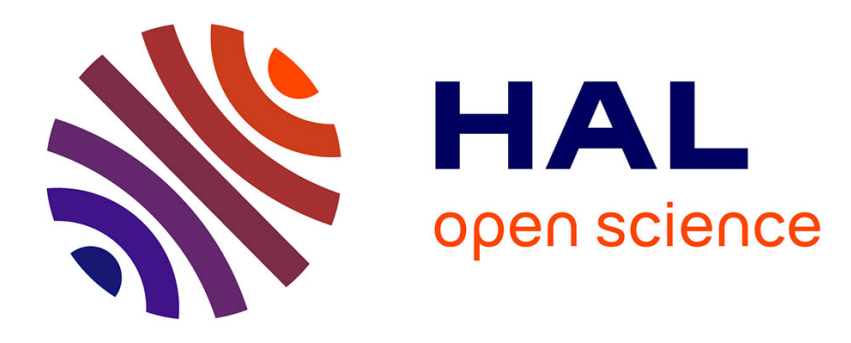

\title{
High-order harmonic and attosecond pulse generation on plasma mirrors: basic mechanisms
}

Cédric Thaury, F Quéré

\section{To cite this version:}

Cédric Thaury, F Quéré. High-order harmonic and attosecond pulse generation on plasma mirrors: basic mechanisms. Journal of Physics B: Atomic, Molecular and Optical Physics, 2010, 43 (21), pp.213001. 10.1088/0953-4075/43/21/213001 . hal-00569857

\section{HAL Id: hal-00569857 \\ https://hal.science/hal-00569857}

Submitted on 25 Feb 2011

HAL is a multi-disciplinary open access archive for the deposit and dissemination of scientific research documents, whether they are published or not. The documents may come from teaching and research institutions in France or abroad, or from public or private research centers.
L'archive ouverte pluridisciplinaire HAL, est destinée au dépôt et à la diffusion de documents scientifiques de niveau recherche, publiés ou non, émanant des établissements d'enseignement et de recherche français ou étrangers, des laboratoires publics ou privés. 


\title{
High-order harmonic and attosecond pulse generation on plasma mirrors: basic mechanisms
}

\author{
C. Thaury ${ }^{1,2}$ and F. Quéré ${ }^{1}$ \\ ${ }^{1}$ Service des Photons, Atomes et Molcules, Commissariat l'Energie Atomique, \\ DSM/IRAMIS, CEA Saclay, 91191 Gif sur Yvette, France \\ ${ }^{2}$ Centre de Physique Théorique, Ecole Polytechnique, CNRS, 91128 Palaiseau, France
}

\begin{abstract}
When an intense femtosecond laser pulse hits an optically-polished surface, it generates a dense plasma that itself acts as a mirror, known as a plasma mirror. As this mirror reflects the highintensity laser field, its non-linear temporal response can lead to a periodic temporal distortion of the reflected wave, associated to a train of attosecond light pulses, and, in the frequency domain, to the generation of high-order harmonics of the laser. This paper presents detailed theoretical and numerical analysis of the two dominant harmonic generation mechanisms identified so far, Coherent Wake Emission and the Relativistic Oscillating Mirror. Parametric studies of the emission efficiency are presented in these two regimes, and the phase properties of the corresponding harmonics are discussed. This theoretical study is complemented by a synthesis of recent experimental results, which establishes that these two mechanisms indeed dominate harmonic generation on plasma mirrors.
\end{abstract}

PACS numbers: 42.65.Ky, 52.65.Rr, 52.27.Ny, 52.38.-r

\section{Contents}

\section{Introduction}

II. Physical properties of plasma mirrors and coupling with the laser field

A. Physical properties: orders of magnitude

B. Brunel vs. resonance absorption

III. Theoretical framework of HHG

A. Conversion to the boosted frame

B. Wave equation with sources in one-dimensional problems

C. Conversion to the reciprocal space

D. Transverse current in a plasma mirror

E. Sources of harmonic generation

F. Particle In Cell codes

IV. Coherent Wake Emission

A. Brunel absorption

B. Crossings of trajectories and formation of a peak of electron density

C. Excitation of plasma oscillations

1. Analysis in the boosted frame

2. Analysis in the laboratory frame

D. Emission of attosecond XUV pulses

1. Observation in PIC simulations

2. Analysis using the transverse current distribution

E. Parametric study

1. Laser intensity

2. Density gradient

3. Incidence angle

F. Phase properties

1. Emission time.

2. Attosecond chirp.

3. Femtosecond chirp and spatial phase.

G. Conclusion
V. Relativistic Oscillating Mirror

A. Observation in PIC simulations 20

B. Mirror in uniform motion 21

C. Doppler effect and retarded currents 21

D. Doppler effect in reciprocal space 22

E. Doppler effect in HHG 23

F. The $\gamma$-spikes model 25

1. Summary and main results 25

2. Limitations of the theory 25

G. Parametric study 26

1. Laser intensity 26

2. Density gradient scale length 27

3. Incidence angle 27

4. Comparison CWE/ROM 28

H. Phase properties 28

I. Conclusion 29

VI. Experimental evidence 30

A. Experimental tools 30

B. Spectral extension of the harmonic signal 31

1. Case of Coherent Wake Emission 31

2. Case of Doppler harmonic generation $\quad 32$

C. Laser intensity dependence 32

1. Case of Coherent Wake Emission 32

2. Case of Doppler harmonic generation 33

D. Spectral width of individual harmonics 33

E. Divergence of the harmonic beam 34

F. Summary and comparison to numerical simulations

Acknowledgments $\quad 36$

$\begin{array}{ll}\text { References } & 36\end{array}$ 


\section{INTRODUCTION}

High-order harmonic generation (HHG) through nonlinear interaction of intense laser beams with different systems has been a subject of strong scientific interest for more than three decades. One of the main motivations that drive such studies is of course fundamental: this generation is generally the signature of a highly non-linear interaction, on which the harmonic signal provides quite direct information. Another important one is to exploit this phenomenon to obtain collimated beams of coherent light at short wavelengths. Generating harmonic orders of a few hundreds of ultrashort visible or near-visible pulses could provide ultrashort light pulses in the soft X-ray range -a goal to which considerable scientific efforts are devoted, due to its huge potential for probing the ultrafast dynamics of matter [1].

In the last decade or so, the interest of the scientific community for HHG became even stronger, as it was realized that this process could be used not only to extend the spectral range of coherent light sources, but also to reduce their pulse duration [2]. Indeed, if many harmonics are generated, a very broad electromagnetic spectrum -i.e. up tens or hundreds of $\mathrm{eV}$ wide- is obtained, which can sustain light pulse durations down to the attosecond range $\left(1 \mathrm{as}=10^{-18} \mathrm{~s}\right)$. Such pulses open the route to directly resolving, in the time domain, the ultrafast dynamics of electrons in atoms, molecules or even solids [3]. Achieving such durations however requires the harmonics to have an appropriate phase relationship.

Since 2001, many experiments have proved that such an appropriate phase relationship can be achieved for harmonics generated in atomic or molecular gases at laser intensities of $10^{14}-10^{15} \mathrm{~W} / \mathrm{cm}^{2}$ in the near-infrared [3$6]$. The present state-of-the-art enables the generation of single attosecond pulses of duration down to 80 as, with photon and pulse energies in the $100 \mathrm{eV}$ and $\mathrm{nJ}$ ranges, respectively $[7,8]$. This remarkable light source, which basic physics is now very well-understood, has already been used in a few pioneering time-resolved experiments on the dynamics of electrons in atoms $[9,10]$ or solids [11]. However, this source is approaching its limits, be it in terms of photon energy, pulse energy or duration, thus hindering the developement of attosecond science.

All these limits on the performance of gas HHG sources can eventually be traced back to the fact that this process totally breaks down at intensities beyond a few $10^{15}$ $\mathrm{W} / \mathrm{cm}^{2}$. This is mostly due to the strong ionization of the generation medium that is induced by the laser field at higher intensities, leading both to a depletion of the individual dipoles responsible for the emission, and to an extremely poor phase matching of these dipoles in the macroscopic generation medium. To avoid this limitation, the next generations of attosecond light sources will either be based on other HHG processes, compatible with the much higher laser intensities of up to $10^{22}$ $\mathrm{W} / \mathrm{cm}^{2}$ now delivered by ultrashort lasers [12], or rely on approaches totally different from HHG -e.g. free elec- tron lasers [13], or relativistic flying mirrors [14-16]. In the former case, the interaction leading to HHG will unavoidably involve ionized media, i.e. plasmas.

Interestingly, the very first experiments where HHG of an intense laser beam was observed already involved interactions with plasmas. After the observation of harmonics orders up to 11 by Burnett et al. in 1977 [17], Carman et al. observed harmonic orders up to 27 [18], and then 49 in a second experiment [19], in the early 80 's. These pioneering experiments used intense nanosecond $\mathrm{CO}_{2}$ lasers $\left(\lambda_{L}=10.6 \mu \mathrm{m}\right)$ focused on initially solid targets, which were turned into plasmas during the interaction. Although these early experiments arose a strong interest in the scientific community because of the unprecedently high generated harmonic orders, they were only followed by a few theoretical studies [20, 21], and to the best of our knowledge, no detailed experimental study. The next significant experimental and theoretical works came only almost 15 years later, and still at a fairly low pace [22]. An important step in this period was provided by the first HHG experiments on solids using tabletop lasers delivering ultrashort (100 fs) pulses [23, 24]. In the meantime though, the focus had switched to HHG in gases, which corresponded to a more accessible interaction regime with the laser technology of the time.

In the past few years, research activity on HHG in plasmas has quickly increased both experimentally and theoretically, thanks to progresses in laser performance and numerical simulation tools, that have respectively resulted in transitions from nanosecond to femtosecond pulse durations for ultraintense lasers, and from megaflops to multi-teraflops capabilities for computing resources. This has led to very significant progresses in the understanding of the basic mechanisms involved [2532 , and to the first experimental evidence for the generation of attosecond pulses of light using plasmas [33].

The most common and successful way to generate harmonics in plasmas so far consists in focusing intense ultrashort -typically a few tens of femtoseconds- laser pulses on initially-solid targets. This target is quickly ionized by the laser field in the early part of the pulse. In the femtosecond regime, plasma expansion can be very limited during the interaction, and the laser pulse then reflects on a dense plasma with a extremely steep interface with vacuum. We shall refer to this system, which characteristics will be defined more precisely in this paper, as a 'plasma mirror'.

Driving $\mathrm{HHG}$ on plasma mirrors typically requires laser intensities beyond a few $10^{15} \mathrm{~W} / \mathrm{cm}^{2}$ for an $800 \mathrm{~nm}$ field [32], that is to say normalized vector potentials $a_{0}=$ $e E / m \omega_{L} c=\left[I\left(W / \mathrm{cm}^{2}\right) \lambda_{L}^{2}\left(\mu m^{2}\right) /\left(1.37 \times 10^{18}\right)\right]^{1 / 2} \gtrsim$ 0.03 . In this expression, $e$ and $m$ are respectively the electron charge and mass, $c$ the vacuum light velocity, $E$ the amplitude of the laser electric field and $I$ the corresponding laser intensity, $\omega_{L}$ the laser frequency, and $\lambda_{L}$ its wavelength.

Two dominant generation mechanisms have now been clearly identified for HHG on plasma mirrors in the case 
of ultrashort laser pulses: these are on the one hand, Coherent Wake Emission (CWE) [32], and on the other hand, the Relativistic Oscillating Mirror (ROM) process [27]. As illustrated by the numerical results of Fig. 1, these two mechanisms lead to very different distorsions of the reflected field, and to very different harmonic spectra.
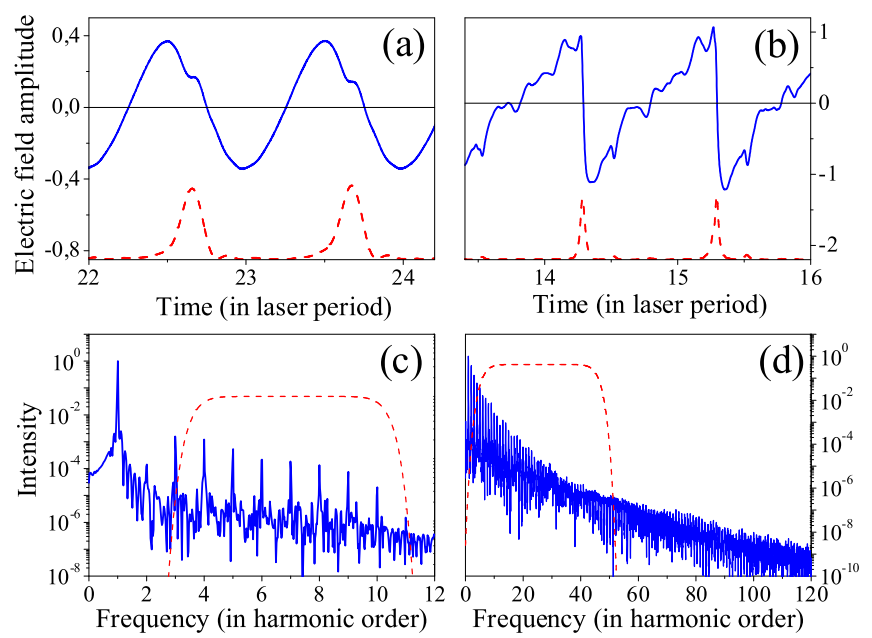

FIG. 1: (Color online) Particle-in-Cell simulations of HHG on plasma mirrors. (a-b) Electric field $E(t)$ of the light reflected by a plasma mirror, in a case where CWE dominates harmonic generation (panel $\left.(\mathrm{a}), a_{0}=0.2, \omega_{p}^{\max }=10 \omega_{L}\right)$, and in a case where the ROM process dominates (panel (b), $a_{0}=5, \omega_{p}^{\max }=$ $\left.15 \omega_{L}\right)$. The corresponding spectra are respectively displayed in panels (c) and (d). The incident laser field on the target was perfectly sinusoidal. The red dashed curves in (a) and (b) show the intensity profiles of the trains of attosecond pulses obtained by filtering groups of harmonics in the spectra, using the spectral filters shown in red dashed lines in (c) and (d).

CWE is a mechanism that qualitatively consists of three main steps:

(i) Electrons at the plasma surface are pulled out in vacuum by the laser field, and then pushed back into the dense plasma after having gained energy from the field.

(ii) These fast electrons propagating in the dense part of the plasma form ultrashort bunches, which impulsively excite plasma oscillations in their wake.

(iii) In the inhomogeneous part of the plasma formed by the density gradient at the plasma vacuum interface, these collective electron oscillations radiate light at the different local plasma frequencies found in this gradient.

Since this process occurs periodically once every laser optical cycle, the spectrum of the associated light emission consists in harmonics of the laser frequency. This harmonic spectrum can extend up to the maximum plasma frequency $\omega_{p}^{\max }$ of the laser-induced plasma (Fig. 1(c)), which typically corresponds to harmonics $15-30$ of an
$800 \mathrm{~nm}$ laser field. CWE generally totally dominates the harmonic signal from plasma mirrors for $a_{0} \lesssim 1$.

At higher intensities, dense outgoing jets of electrons at the plasma surface are accelerated by the laser field up to relativistic velocities. These moving distribution of charges induce a transient Doppler frequency upshift on the laser field reflected by the dense plasma. Since this Doppler effect again occurs periodically at the laser frequency, this leads to a spectrum consisting of laser harmonics (Fig. 1(d)). It turns out that ROM is the dominant source of harmonic generation beyond $\omega_{p}^{\max }$, while below this frequency, both CWE and ROM can contribute to the harmonic signal, and their relative weight depends on laser intensity.

The goal of this paper is to present detailed numerical and theoretical analysis of these two generation mechanisms. CWE has been identified only recently, and this paper thus provides the first detailed analysis and parametric study of this process. Regarding the ROM mechanism, the novelty brought by this paper is a new and simple way of analyzing the laser-induced electric currents in the plasma responsible for harmonic emission. This provides a new insight into the most essential feature of this process, and can be used to diagnose the occurrence of a Doppler effect in numerical simulations. In addition, we present one of the most complete parametric study of ROM so far, and confront the results to the most recent analytical theory of this process.

The outline of the paper is the following. In Sec. II we discuss the main physical features of plasma mirrors, and the coupling mechanisms with the laser field. The basic theoretical framework needed to analyze HHG in plasmas is then presented in Sec. III. Section IV is a detailed numerical and theoretical analysis of CWE, while Sec. V is devoted to the study of ROM. Finally, Sec. VI gathers several experimental observations which support the existence of these two generation mechanisms.

\section{PHYSICAL PROPERTIES OF PLASMA MIRRORS AND COUPLING WITH THE LASER FIELD}

In this section, we discuss the main physical characteristics of plasmas created by intense ultrashort laser pulses interacting with initially-solid targets. We thus define more precisely what is meant by the term 'plasma mirror', and then point out the main coupling mechanism of such plasmas with intense laser fields.

\section{A. Physical properties: orders of magnitude}

The most appropriate tools to simulate the transition from the solid to the plasma states are hydrodynamic codes, because these codes take into account collisional absorption of the laser field -which plays a major role at the moderate laser intensities where the plasma is created 
$\left(10^{14}-10^{16} \mathrm{~W} / \mathrm{cm}^{2}\right)$ - as well as the ionization of the target following the resulting energy deposition. Figure 2 shows the results of such an hydrodynamic simulation performed with the code MULTI-fs [34, 35], for a $60 \mathrm{fs}$ laser pulse of $10^{17} \mathrm{~W} / \mathrm{cm}^{2}$ intensity impinging a bulk aluminum target $\left(Z_{\max }=13\right)$ with a $45^{\circ}$ incidence angle in $p$-polarization.

These results demonstrate that plasmas created on solid targets by ultrashort laser pulses (with high enough temporal contrasts, see Sec. VI) hardly have time to expand during the laser pulse (Fig. 2(a)). The laser-plasma interaction thus involves a sharp density gradient at the plasma-vacuum interface. In addition, they show that even at the moderate intensity of this simulation, the surface of this low- $Z$ target is almost fully ionized before the peak of the pulse (Fig. 2(b)).

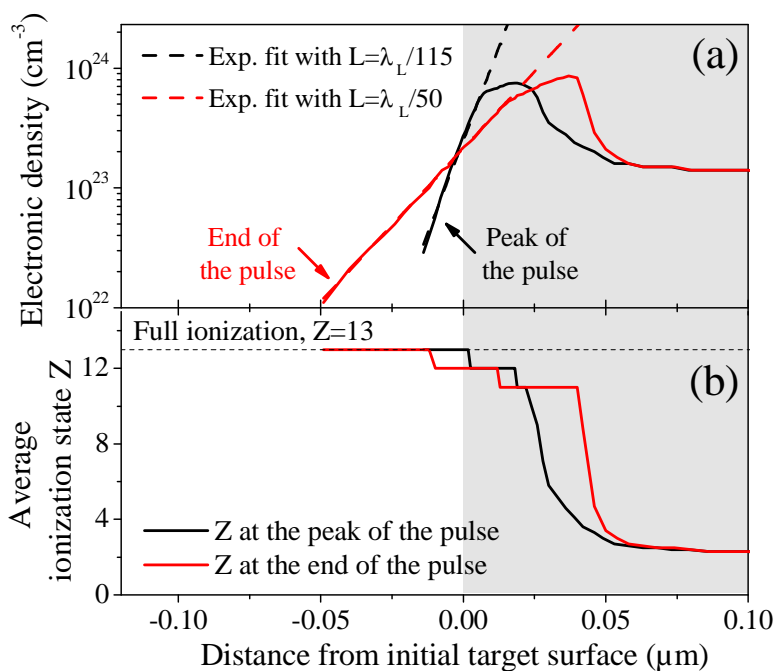

FIG. 2: (Color online) Hydrodynamic simulation using the MULTI-fs code, in the case of aluminum for a 60 fs long Gaussian laser pulse in $p$-polarization and a peak intensity of $10^{17}$ $\mathrm{W} / \mathrm{cm}^{2}$. The laser wavelength is $800 \mathrm{~nm}$. The initial solid target occupies the half space $x \geq 0$, highlighted in gray.

As a result of the limited expansion and the almost complete ionization, the electron density of the plasma created during the rising edge of the pulse on a low$\mathrm{Z}$ target is of the order of the initial solid target total electron density. Depending on the material used, this leads to typical ion and electron densities in the $200 n_{c}$ (plastic target) to $400 n_{c}$ (silica, aluminum) range, where $n_{c}=m \epsilon_{0} \omega_{L}^{2} / e^{2} \approx 1.7 \times 10^{21} \mathrm{~cm}^{-3}$ is the critical density for an $800 \mathrm{~nm}$ laser field. In contrast, targets of high $Z$, such as gold, are unlikely to be fully ionized even at relativistic laser intensities. However, given the limited expansion, the plasma density is still expected to be a significant fraction of the solid target total electron density in these cases.

Due to its highly over-critical density, this plasma efficiently reflects the incident laser field. Besides, thanks to the very limited expansion during the laser pulse, the density gradient scale length at the center and the edges of the focal spot can only differ by a small fraction of the laser wavelength, so that the plasma-vacuum interface has a high flatness. This plasma thus has the ability to efficiently reflect the incident laser pulse with a negligible distortion of its wavefront. These characteristics define what we call a plasma mirror (PM). At high enough intensities, the non-linear temporal response of such a PM to the incident field however leads to a periodic temporal distortion of the reflected waveform, resulting in the harmonic generation analyzed in this paper.

\section{B. Brunel vs. resonance absorption}

The most efficient way to drive HHG on plasma mirrors is generally to use a laser field in oblique incidence and $p$-polarization, i.e. with the electric field of the laser that lies in the incidence plane, and has a component $E_{x}$ normal to the plasma surface. In these conditions, the coupling with the laser field can involve different kinetic absorption processes, two of which have been wellidentified so far.

The first mechanism is the widely-documented resonance absorption [36]. At low laser intensities, this is a linear mode conversion process, by which a fraction of the laser energy is converted into electrostatic energy, in the form of plasma oscillations, excited at the point $x_{A R}$ of the plasma density gradient where the local frequency $\omega_{p}(x)=\sqrt{n_{e}(x) e^{2} / m \epsilon_{0}}$ of these modes matches the laser frequency $\omega_{L}$. For a given incidence angle $\theta$, there is an optimal gradient scale length $L_{o}$, independent of laser intensity, that maximizes resonance absorption [37].

As the laser intensity increases, the excursion amplitude $d$ of the plasma electrons exposed to the laser field becomes so large that it exceeds the gradient scale length $L$. In this regime, Brunel stressed that resonance absorption is no longer relevant, even when the gradient scale length matches the intensity-independent optimum $L_{o}$ for resonance absorption. He then analyzed a new absorption mechanism, now often called Brunel absorption [38]. In this mechanism, electrons at the plasma surface are first pulled out of the plasma by the laser field, when $E_{x}$ points inward, and then evolve under the effect of this laser field and the electrostatic space charge field. As the total electric field changes sign, the majority of these electrons are pushed back into the dense plasma, where they escape the effect of the laser field due to plasma screening. They thus carry away into the target the energy they gained during their excursion into vacuum, leading to absorption of laser energy.

Resonance and Brunel absorptions in fact correspond to two extreme regimes of the laser-plasma coupling dynamic, which can be analyzed more generally in terms of anharmonic resonance [39]. The coupling progressively evolves from pure resonance absorption, for smooth density gradient and/or moderate laser intensities, to pure Brunel absorption, for sharper gradients and/or higher laser intensities. An essential question here is to know 
which of these two limiting regimes, if any, is more appropriate in the case of HHG on plasma mirrors.

Brunel's criterion is based on comparing the electron excursion $d$ and the gradient scale length $L$ [38]. In a crude approximation, one can use $d \approx v_{\text {osc }} / \omega_{L}$, with $v_{\text {osc }}$ the typical quivering velocity of electrons in the field. Further assuming that $v_{o s c} \approx e E / \gamma m \omega_{L}=a_{0} c / \gamma$, with $\gamma=\sqrt{1+a_{0}^{2}}$ the electron Lorentz factor, we obtain an estimation of the excursion

$$
\frac{d}{\lambda_{L}} \simeq \frac{a_{0}}{2 \pi \sqrt{1+a_{0}^{2}}}
$$

Figure 3 shows as a black line in the $(I, L)$ parameter space (i.e. laser intensity and gradient scale length), the locations where the excursion $d$ given by Eq. (1) equals the gradient scale length $L$. Brunel-like absorption is expected in the lower area, while resonance absorption is more relevant in the upper one.

By considering this graph and the results of hydrodynamic simulations presented in Sec. II A, it appears that HHG on plasma mirrors will in most cases occur in a regime where the Brunel mechanism provides the most appropriate description of the laser energy absorption. This crude analysis is well supported by numerical simulations.

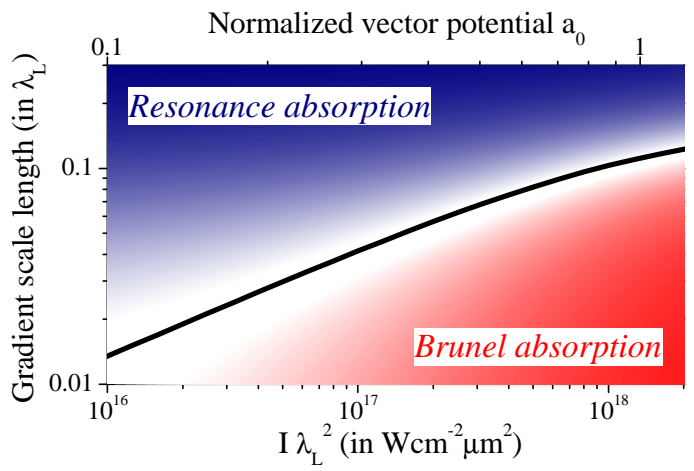

FIG. 3: (Color online) Transition from resonance to Brunel absorption as a function of laser intensity $I$ and gradient scale length $L$. The black line shows the locations where the typical electron excursion $d$ given by Eq. (1) equals $L$.

\section{THEORETICAL FRAMEWORK OF HHG}

In this section, we introduce the basic equations and concepts required to analyze HHG from plasma mirrors, and discuss how the currents driven by the laser field in the plasma can generate high-order harmonics. We consider a plasma mirror which occupies the half-space $x \geq 0$, and an observation point located in vacuum, in front of this target.
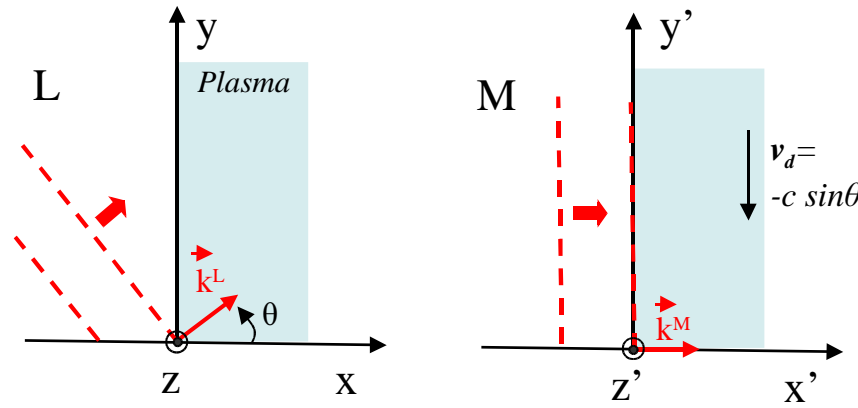

FIG. 4: (Color online) Transformation from the laboratory frame L to the boosted frame M.

\section{A. Conversion to the boosted frame}

The interaction of a flat overdense plasma target with an electromagnetic plane wave in oblique incidence is a two dimensional (2D) problem in the laboratory frame. However, it can be converted into a more tractable 1D problem by using a Lorentz transformation to a reference frame moving along the target surface [40]. This wellknown transformation to the so-called boosted frame is widely used both in PIC codes and analytical studies.

Let $\mathbf{y}$ be the unit vector defined by the intersection of the incidence plane and the target surface, and $\theta$ the angle of incidence of the laser field on the plasma (Fig. 4). The boosted frame $M$ moves with a velocity $c \sin \theta \mathbf{y}$ along the target surface, corresponding to relativistic factors $\beta=\sin \theta$ and $\Gamma=1 / \sqrt{1-\beta^{2}}=1 / \cos \theta$.

The vacuum angular frequency and wave vector of the laser field in the laboratory frame $L, \omega^{L}$ and $k^{L}=$ $\left(\omega^{L} \cos \theta / c, \omega^{L} \sin \theta / c, 0\right)$, transform to $\omega^{M}=\omega^{L} \cos \theta$ and $k^{M}=\left(\omega^{L} \cos \theta / c, 0,0\right)$. In frame $M$, the laser field thus impinges the plasma in normal incidence, and the whole plasma has a drift velocity $\mathbf{v}_{d}=-c \sin \theta \mathbf{y}$ along the surface.

The electron and ion densities of the plasma, $n_{e}^{L}(x)$ and $n_{i}^{L}(x)$ in the laboratory frame change by a factor $\Gamma$ when going to the moving frame, i.e. $n_{e}^{M}(x)=n_{e}^{L}(x) / \cos \theta$ and $n_{i}^{M}(x)=n_{i}^{L}(x) / \cos \theta$.

\section{B. Wave equation with sources in one-dimensional problems}

Using this Lorentz transformation to reduce the problem to one spatial dimension (the $x$ axis), a simple expression of the field radiated by the plasma currents can be obtained. We describe the electromagnetic field using the scalar and vector potentials in the Coulomb jauge, defined by the condition $\nabla . \mathbf{A}=0$. In this gauge, radiation fields are entirely determined by the vector potential A, which satisfies the wave equation:

$$
\nabla^{2} \mathbf{A}-\frac{1}{c^{2}} \frac{\partial^{2} \mathbf{A}}{\partial t^{2}}=-\mu_{0} \mathbf{j}_{t}
$$


where $\mathbf{j}_{t}$ is the transverse current, such that the total current $\mathbf{j}=\mathbf{j}_{l}+\mathbf{j}_{t}$ with $\nabla \cdot \mathbf{j}_{t}=0$ and $\nabla \times \mathbf{j}_{l}=\mathbf{0}$.

The transverse current $\mathbf{j}_{t}$ is in general a complex and non-local function of $\mathbf{j}$ (see Eq. (6.28) in Ref. [41]). However, its expression becomes very simple in onedimensional problems, where $\mathbf{j}_{t}=\left(0, j_{y}, j_{z}\right)$. Using the boosted frame to study HHG from plasma mirrors is thus particularly convenient.

In one dimension, a Green's function for the wave equation Eq. (2) is simply $G\left(x_{0}-x, t_{0}-t\right)=$ $\Theta\left[t_{0}-t-\left|x_{0}-x\right| / c\right]$, where $\Theta$ is the Heaviside function $(\Theta(t)=1$ for $t \geq 0$, and $\Theta(t)=0$ for $t<0)$. This leads to the following general solution for the vector potential $\mathbf{A}^{r}$, induced at point $x_{0}$, by a transverse current distribution $\mathbf{j}_{t}(x, t)$ located in the half space $x>x_{0}$ (see Fig. 5):

$$
\mathbf{A}^{r}\left(x_{0}, t_{0}\right)=-\mu_{0} \int_{x_{0}}^{+\infty} d x \int_{-\infty}^{t_{0}-\left(x-x_{0}\right) / c} d t \mathbf{j}_{t}(x, t) .
$$

We emphasize that this expression only corresponds to the radiation by the plasma. It does not include the vector potential $\mathbf{A}^{i}$ of the incident laser field which is a solution of the homogeneous differential equation obtained by setting $\mathbf{j}_{t}=0$ in Eq. (2).

In $1 \mathrm{D}$, the transverse (or radiation) electric field is $\mathbf{E}_{t}=-\partial \mathbf{A} / \partial t$. Applying this last relationship to Eq. (3) leads to the following expression for the transverse electric field:

$$
\mathbf{E}^{r}\left(x_{0}, t_{0}\right)=\mu_{0} \int_{x_{0}}^{+\infty} d x \mathbf{j}_{t}\left[x, t_{0}-\left(x-x_{0}\right) / c\right] .
$$

In this expression, $t_{r e t}=t_{0}-\left(x-x_{0}\right) / c$ is the retarded time, which corresponds to the emission time of the signal originating from location $x$ and arriving at location $x_{0}$ at time $t_{0}$. The field radiated by the plasma is thus simply obtained by integrating the transverse current along a line of slope $c$ in the $(x, t)$ space of the boosted frame, i.e. along a 1D light 'cone', as illustrated in Fig. 5.

\section{Conversion to the reciprocal space}

What is generally measured experimentally is $\left|\hat{\mathbf{E}}^{r}\left(x_{0}, \omega\right)\right|^{2}$ for a position $x_{0}$ in vacuum, where $\hat{\mathbf{E}}^{r}\left(x_{0}, \omega\right)$ is the Fourier transform of $\mathbf{E}^{r}\left(x_{0}, t\right)$ with respect to time. Since this spectrum is independent of position in vacuum for a $1 \mathrm{D}$ system, we now note this function $S(\omega)=\left|\hat{\mathbf{E}}^{r}\left(x_{0}, \omega\right)\right|^{2}$. By Fourier-transforming Eq. (4) with respect to time, the following expression is obtained for $S(\omega)$ :

$$
\begin{aligned}
S(\omega) & =\mu_{0}^{2}\left|\int_{x_{0}}^{+\infty} d x \hat{\mathbf{j}}_{t}(x, \omega) e^{-i \omega x / c}\right|^{2} \\
& =\mu_{0}^{2}\left|\hat{\hat{\mathbf{j}}}_{t}(k=\omega / c, \omega)\right|^{2},
\end{aligned}
$$

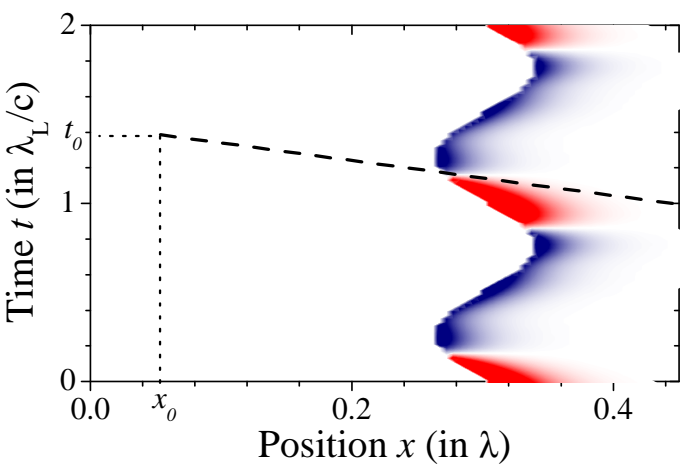

FIG. 5: (Color online) Illustration of the calculation of the electric field radiated on one side of an arbitrary 1D transverse current distribution $j_{t}$ (red to blue color map) with the retarded current integral. The electric field radiated at position $x_{0}$ and time $t_{0}$ is proportional to the integral of $j_{t}$ along the dashed line, of slope $c$ in this $(x, t)$ space. Red and blue corresponds to different signs of $j_{t}$.

where $\hat{\mathbf{j}}_{t}(x, \omega)$ is the Fourier transform of $\mathbf{j}_{t}(x, t)$ with respect to time, and $\hat{\hat{\mathbf{j}}}_{t}(k, \omega)$ the Fourier transform of $\mathbf{j}_{t}(x, t)$ with respect to both time and space. This equation shows that the emitted spectrum is simply given by a lineout of $\left|\hat{\mathbf{j}}_{t}(k, \omega)\right|^{2}$ along an oblique line $k=\omega / c$ in the $2 \mathrm{D}$ reciprocal space $(k, \omega)$.

To analyze the Doppler effect induced by moving current distributions, which will be studied in Sec. V, it is useful to introduce the mathematical concept of instantaneous field $\tilde{E}(t)$, which is the field that would be radiated by the same current distribution $\mathbf{j}_{t}(x, t)$ in the absence of retardation effects, i.e. by taking the limit $c \rightarrow \infty$ in Eq. (4):

$$
\tilde{\mathbf{E}}(t)=\mu_{0} \int_{x_{0}}^{+\infty} d x \mathbf{j}_{t}(x, t) .
$$

The corresponding instantaneous spectrum $\tilde{S}(\omega)$, defined as the intensity of the Fourier transform of $\tilde{\mathbf{E}}(t)$, is given by

$$
\tilde{S}(\omega)=\mu_{0}^{2}\left|\hat{\mathbf{j}}_{t}(k=0, \omega)\right|^{2}
$$

The instantaneous spectrum $\tilde{S}(\omega)$ thus corresponds to a lineout of $\left|\hat{\hat{j}}_{t}(k, \omega)\right|^{2}$, along the $\omega$ axis in the 2 D reciprocal space $(k, \omega)$.

In the following, we temporarily turn to a fluid model of the plasma, to get a simple expression of the transverse current. Then, we discuss the different possible origins of harmonics generated from plasma mirrors.

\section{Transverse current in a plasma mirror}

It has been demonstrated in Ref. [27] that, in a fluid model of the plasma, the transverse current $\mathbf{j}_{t}$ in the 
boosted frame can be expressed analytically as a function of the total vector potential $\mathbf{A}$ and the fluid velocity along the target normal. In this section, we discuss this relationship to emphasize the physical origin of the different terms.

The response of the ions to the laser field is assumed to be negligible. These ions, of charge $Z e$, nevertheless contribute to the transverse current because of the plasma drift along $\mathbf{y}$ at $\mathbf{v}_{d}=-c \sin \theta \mathbf{y}$ :

$$
\begin{aligned}
\mathbf{j}_{t}^{i} & =-Z e n_{i}^{M}(x, t) c \sin \theta \mathbf{y} \\
& =-Z e n_{i}^{L}(x, t) c \tan \theta \mathbf{y} .
\end{aligned}
$$

The electron contribution to the transverse current is obtained by using the conservation of the component of the generalized momentum in the target surface plane, which results from the translational invariance of the system in this plane:

$$
\mathbf{p}_{t}-e \mathbf{A}=\text { const. }=\mathbf{p}_{t}^{0}
$$

where $\mathbf{p}_{t}=m \gamma \mathbf{v}_{t}$ is the transverse momentum of the electron fluid, $\gamma=\left(1-\beta_{x}^{2}-\beta_{t}^{2}\right)^{-1 / 2}$ is the Lorentz factor of the electron fluid, and $\mathbf{p}_{t}^{0}=-m c \tan \theta \mathbf{y}$ is the value of the momentum before the laser arrives $(\mathbf{A}=0)$. The electronic part of the transverse current is then given by $\mathbf{j}_{t}^{e}=-e n_{e}^{M} \mathbf{v}_{t}=-e n_{e}^{L} \mathbf{p}_{t} / m \gamma \cos \theta$.

Using Eqs. (8) and (9) leads to the following expression for the total transverse current $\mathbf{j}_{t}=\mathbf{j}_{t}^{e}+\mathbf{j}_{t}^{i}$ :

$$
\begin{aligned}
\mathbf{j}_{t}(x, t) & =-\frac{e^{2} n_{e}^{L}(x, t)}{m \cos \theta} \frac{\mathbf{A}(x, t)}{\gamma(x, t)} \\
& -e c \tan \theta\left[Z n_{i}^{L}(x, t)-\frac{1}{\cos \theta} \frac{n_{e}^{L}(x, t)}{\gamma(x, t)}\right] \mathbf{y},
\end{aligned}
$$

with

$$
\gamma(x, t)=\sqrt{\frac{1+(e \mathbf{A} / m c-\tan \theta \mathbf{y})^{2}}{1-\beta_{x}^{2}}} .
$$

According to Eq. (10), two terms contribute to the transverse current in the plasma, and hence to the reflected field. The first term of the right-hand side of Eq. (10), proportional to $\mathbf{A}$, is the current directly driven by the total radiation field. In the following, it is referred to as the conduction current. The origin of the second term is more complex. This current is induced because of the drift of the plasma in the boosted frame when $\theta \neq 0$, and appears as soon as there is a difference between the density distributions of ions and electrons. This can occur either (i) because $n_{i}^{L}(x, t) \neq n_{e}^{L}(x, t)$, i.e. the electron fluid has moved or has been distorted compared to the ion fluid, or (ii) because $\gamma(x, t) \neq 1 / \cos \theta$, $i . e$. the electron fluid has been accelerated by the laser field, thus leading to a change of its density by relativistic compression.

The physical meaning of this second term becomes clearer in the laboratory frame. Let us assume that the incident laser field induces a perturbation of the electron density at a position $y_{0}$ along the target surface at time $t_{0}$, resulting in a non-vanishing local total charge density $\rho\left(x, y_{0}, t_{0}\right)$. In oblique incidence, this perturbation propagates with a phase velocity $c / \sin \theta$ along the target surface, and will thus occur at a different time $t=t_{0}+\left(y-y_{0}\right) \sin \theta / c$ at a different point $y$ of the surface. The resulting moving charge density $\rho(x, y, t-y \sin \theta / c)$ leads to a current along the $y$ axis, which physically corresponds to the second term on the right-hand side of Eq. (10).

Since this term only occurs for $\theta \neq 0$, we call it the obliquity current. Note that this current is always along the $y$ axis. This means that even for an $s$-polarized incident field $(\mathbf{E} \| z)$, some $p$-polarized light can be radiated by the plasma.

\section{E. Sources of harmonic generation}

We now discuss the different possible ways in which harmonics of the incident laser frequency can be generated by the plasma, using the fluid model presented in Sec. III D.

We first use a perturbative approach, by assuming that the total vector potential A in Eq. (10) is perfectly sinusoidal, i.e. that the new frequencies introduced in $\mathbf{A}$ by the plasma response correspond to small corrections and can be neglected. Two effects can then lead to periodic temporal modulations of $\mathbf{j}_{t}$ compared to $\mathbf{A}$, and hence to the generation of harmonic frequencies in $\mathbf{E}^{r}$ :

- $\gamma(x, t)$ can modulate both the conduction and obliquity currents. In the case of the conduction current, this effect simply accounts for the saturation of the electron velocity when $\left|\mathbf{v}_{t}\right| \rightarrow c$. This saturation obviously leads to a non-sinusoidal transverse current in the plasma, and hence to the generation of new frequencies. This has been called the 'sliding mirror' effect [42].

- The electron density $n_{e}^{L}(x, t)$ can also introduce new frequencies in $\mathbf{j}_{t}$. Temporal variations of $n_{e}^{L}(x, t)$ directly modulate the conduction current, and also leads to a non-vanishing obliquity current. This second effect plays a crucial role in Coherent Wake Emission, due to large electron density modulations induced by plasma oscillations (see section IV).

This analysis highlights the factors that can generate new frequencies in the local current $\mathbf{j}_{t}(x, t)$, at a given position $x$. This is however not enough to determine the spectrum of the emitted light $S(\omega)$, which is related to the whole spatio-temporal structure of $\mathbf{j}_{t}(x, t)$ through Eq. (5). In particular, we will see in Sec. V that for relativistic motions of the plasma, new frequencies can be induced in $S(\omega)$, due to a Doppler effect.

Finally, we have so far assumed that $\mathbf{A}$ is not significantly distorted compared to the vector potential $\mathbf{A}^{i}$ of 
the incident laser. However, since $\mathbf{A}$ is the total vector potential, it includes the distortion induced on the laser field by the plasma response, and might thus not be sinusoidal. This directly introduces new frequencies in the conduction current. To first order, these new frequencies simply correspond to the linear conduction current induced by harmonics as they propagate into the plasma from their generation point to vacuum. To higher order, these new terms can also be modulated by the same effects as discussed above (i.e. by the $\gamma$ and $n_{e}^{L}$ terms), leading to generation of harmonics of the harmonics. This kind of cascaded harmonic generation is likely to occur at very high laser intensities, where the distortion of the total field compared to the incident field becomes extremely strong, and a perturbative approach becomes inappropriate.

The fluid approach provides a formal expression of the transverse current $\mathbf{j}_{t}$, useful for a general analysis. But this expression is most often useless to actually calculate the emission spectrum, since $\mathbf{j}_{t}$ depends on several complex physical functions which are unknown, and which can not be calculated within a fluid model [27]. To obtain this current distribution, it is generally necessary to turn to numerical simulations, in particular using Particle-inCell codes.

\section{F. Particle In Cell codes}

In this section, we briefly present the codes we use to simulate HHG on plasma mirrors, and specify the numerical conditions in which these simulations have been performed.

We use two relativistic collisionless Particle-In-Cell (PIC) codes [43, 44], EUTERPE and CALDER:

- EUterpe was developed in the 80's by Guy Bonnaud [45]. It is a relativistic 1D-3V PIC code, that is to say that it only considers one spatial dimension but calculates the particle velocities in all three spatial directions. To simulate the interaction of a plasma mirror with a plane laser wave in oblique incidence, EUTERPE uses the boosted frame transformation described in Sec. III A.

- CALDER is a relativistic fully-parallelized 3D PIC code, developed by E. Lefebvre in the late 90's [46]. As 3D simulations of plasma mirrors in realistic physical conditions are too time-consuming with currently available computing resources, we typically use this code in 2D3V. Moreover, to reduce the calculation time, we generally simulate the interaction of the plasma with a plane wave, using periodic transverse boundary conditions and a simulation box with a transverse width (along $y$ ) of $\lambda_{L} / \sin \theta$. These typical simulations are however completed by some simulations where the focal spot of the laser pulse is properly taken into account.
Unless otherwise stated, the incident laser pulse is $p$ polarized, has a $\sin ^{2}$ temporal intensity profile, and impinges a plasma with fixed ions, under an incidence angle of $45^{\circ}$. We assume an exponential density gradient of scale length $L$ at the plasma surface, $n_{e}=n_{c} \exp (x / L)$, followed by an homogeneous plasma up to the boundary of the simulation box. Electrons reaching this boundary are re-injected at the same locations with thermal velocities, to simulate the return current in a massive target. In 2D, periodic boundary conditions are used in the transverse direction. Other typical parameters of the simulations are indicated in Tab. I.

\begin{tabular}{lcc}
\hline \hline Code & EUTERPE & CALDER \\
$T_{e 0}(\mathrm{eV})$ & 500 & 500 \\
$T_{i 0}(\mathrm{eV})$ & 100 & 100 \\
$\omega_{L} \Delta x / c$ & $1 \times 10^{-2}$ & $7 \times 10^{-3}$ \\
$\omega_{L} \Delta t$ & $7 \times 10^{-3}$ & $5 \times 10^{-3}$ \\
$\Delta T / T_{L}$ & 20 & 35 \\
\hline \hline
\end{tabular}

TABLE I: Typical conditions of PIC simulations. $T_{e 0}, T_{i 0}$ are respectively the initial electron and ion temperatures, $\Delta x$ is the cell width, $\Delta t$ the time step, and $\Delta T$ the full width of the laser pulse.

\section{COHERENT WAKE EMISSION}

At moderate laser intensities $\left(a_{0} \lesssim 1\right)$, relativistic effects are generally weak, and according to the discussion of Sec. III E, the main source of harmonic emission is the temporal variation of $n_{e}(x, t)$. This type of effect has actually been identified as a potential source of high order harmonics of a laser pulse since 1979 [47]. More recently, a model called Coherent Wake Emission has been proposed to describe precisely the mechanism underlying this emission, in the specific case where the laser interacts with a plasma mirror [32].

This part of the paper provides a detailed description of this process. We first analyze the mechanism of 'Brunel absorption', which has already been introduced in Sec. II B, and plays an essential role in CWE. We then study the different steps of the CWE process, and finally conclude this part by a parametric study and an investigation of the phase properties of the emitted harmonics.

\section{A. Brunel absorption}

To analyze the principle of the Brunel mechanism, we first consider the case of a $p$-polarized laser beam impinging a plasma mirror with a perfectly sharp interface $(L=0)$, which occupies the half-space $x>0$, and we use Brunel's original analytical model of the process [38]. Figure 6 shows the temporal evolution of the positions and velocities of a set of electrons, initially at the surface 
of the plasma and dragged out into vacuum at different instants in a laser optical cycle.

For $0<t<0.25 T_{L}$, the component $E_{x}$ of the total electric field along the target normal pulls electrons out of the plasma. As soon as this electric field component decreases due to the laser field oscillation, the space charge electrostatic component of $E_{x}$ starts pushing a part of these electrons back to the plasma $\left(0.25<t<0.5 T_{L}\right)$. At $t=0.5 T_{L}$, the component of $E_{x}$ corresponding to the laser field changes sign and $E_{x}$ pushes all electrons toward $x>0$. Most of them subsequently return to the overcritical plasma $(x>0)$, where they no longer feel the laser field and keep the velocity they have acquired in vacuum. This process, which repeats itself identically with the periodicity of the laser, can be responsible for a significant absorption of the laser energy.

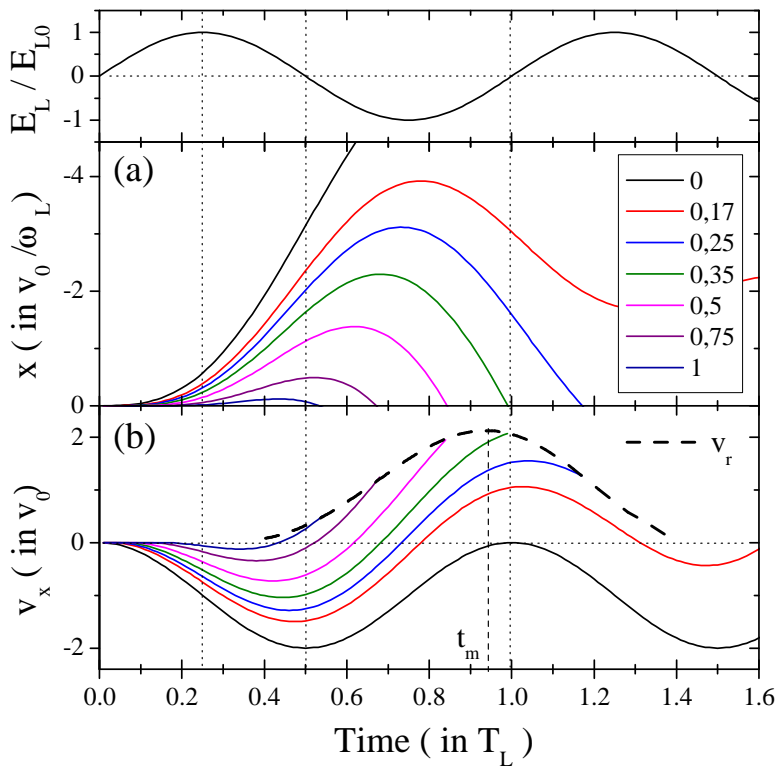

FIG. 6: (Color online) Trajectories and velocities of Brunel electrons calculated using the analytical model described in Ref. [38]. (a) Positions $x(t)$ of 7 electrons along the target normal, as a function of time. The plasma occupies the half space $x>0$. The legend indicates the times $t_{i} / T_{L}$ at which each electron starts to be pulled out of the plasma. (b) Velocities of the same electrons, from $t_{i}$ up to their return time to the plasma $t_{r}$. The dashed line corresponds to the return velocity $v_{r}\left(t_{r}\right)$ of electrons as they reach the plasma boundary. $v_{0}=e E_{0} / m \omega_{L}$ is the quiver velocity of a free electron placed in an oscillating electric field of amplitude $E_{0}$ and frequency $\omega_{L}$

The dashed line in Fig. 6(b) corresponds to the velocity $v_{r}\left(t_{r}\right)$ of Brunel electrons as they return to the plasma, at time $t=t_{r}$. Within Brunel's model, this velocity then remains constant as they propagate in the plasma. The first electrons which come back have been recalled just after having been pulled out, and thus have a velocity $v_{r} \approx 0$. On the contrary, electrons that return around $t_{r}=t_{m} \approx T_{L}$ have been accelerated by the electric field during a long period, and get the maximum return veloc- ity. Those which come back later have been slowed down by the laser field, as it starts pulling electrons toward vacuum again after $t=T_{L}$.

The shape of the velocity distribution $v_{r}\left(t_{r}\right)$, and more specifically the fact that $\partial v_{r} / \partial t_{r}>0$ for $t_{r}<t_{m}$, has a crucial consequence. For $t_{r}<t_{m}$, electrons that have returned to the plasma later eventually catch up electrons that have returned earlier. This leads to a process of trajectory crossing, which is analyzed in the next section.

\section{B. Crossings of trajectories and formation of a peak of electron density}

An essential question to address for the study of trajectory crossing of Brunel electrons inside the plasma, is whether or not their velocity can be considered constant in this area. In his initial model, Brunel assumes that the plasma is a perfect conductor, so that the electric field is exactly 0 inside the plasma, and electrons therefore indeed propagate with a perfectly constant velocity $v_{r}\left(t_{r}\right)$. Without this assumption, Bonnaud et al. showed analytically that for a solid-density plasma with a steplike interface, the residual electric field inside the plasma does not either significantly perturb the electron trajectories [48]. But the case of a density gradient of finite length is much more complex. We therefore turn to PIC simulations to analyze this more realistic situation, using the 1D3V code EUTERPE.

We consider a plasma with a gradient scale length $L=$ $\lambda_{L} / 60$, and a maximum electron density $n_{e}^{\max }=225 n_{c}$, illuminated by a $20 T_{L}$-long laser pulse, with a normalized vector potential $a_{0}=0.2$. Figure 7 represents the evolution in time of the velocity along the target normal $v_{x}$, of eleven electrons, pulled out of the plasma around the

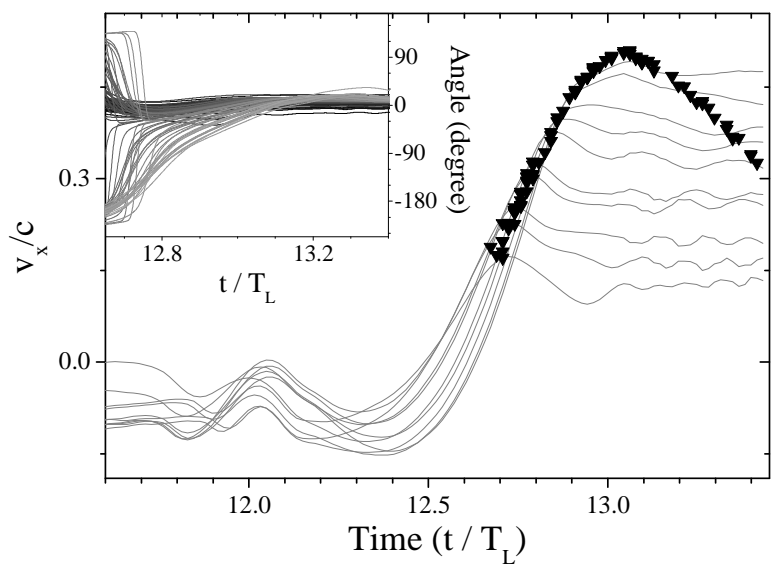

FIG. 7: Velocities and directions of propagation of returning Brunel electrons. The main panel shows the evolution in time of the velocities $v_{x}(t)$ of eleven electrons. The triangles correspond to the velocities $v_{r}\left(t_{r}\right)$ of 100 electrons chosen randomly, at the time $t_{r}$ when they cross the critical density surface. The evolution in time of the propagation angles of these electrons are plotted in the inset. 
maximum of the laser pulse. The triangles on the same figure indicate the velocities of 100 electrons, measured when they cross the critical density surface. From these points, up to the extreme right of the figure, the velocities are almost constant. It is thus a safe assumption to consider that Brunel electrons keep their return velocity $v_{r}\left(t_{r}\right)$ in the overcritical part of the plasma, where $v_{r}\left(t_{r}\right)$ is now their velocity when they cross the critical density surface, at time $t_{r}$.

The inset in Fig. 7 shows that Brunel electrons come back to the plasma with a velocity almost parallel to the target's normal, with propagation angles $-13^{\circ}<\theta_{r}<$ $22^{\circ}$. We also observe that their directions of propagation vary very weakly in the overdense plasma. As a consequence, we can assume that electrons travel across the plasma along its normal, and that the problem is onedimensional.

In order to describe the crossing of trajectories occurring during one laser optical cycle, we approximate the function $v_{r}\left(t_{r}\right)$ for $t_{r}<t_{m}$ by a linear function, $v_{r}\left(t_{r}\right)=\alpha\left(t_{r}-t_{r 0}\right)$, where $t_{r 0}$ is an adjustable parameter used to fit the function $v_{r}\left(t_{r}\right)$ provided by PIC simulations. According to Fig. 7 this assumption is reasonable as long as $t_{r}$ is not too close to $t_{m}$. The trajectory in the overdense plasma of a Brunel electron returning at $t_{r}$ is given, as a function of time $t$ by

$$
x\left(t_{r}, t\right)=\alpha\left(t_{r}-t_{r 0}\right)\left(t-t_{r}\right) .
$$

The electrons which trajectories cross at a given $x=x_{c}$ are those that have returned the plasma around the time $t_{r}$ such that

$$
\left.\frac{\partial t}{\partial t_{r}}\right|_{x=x_{c}}=1-\frac{x_{c}}{\alpha\left(t_{r}-t_{r 0}\right)^{2}}=0 .
$$

This leads to $t_{r}=t_{r 0}+\sqrt{x_{c} / \alpha}$. The position of the crossing point $x_{c}$ as a function of time $t$ is obtained by using this value of $t_{r}$ in Eq. (12), resulting in

$$
x_{c}=\alpha \sqrt{\frac{x_{c}}{\alpha}}\left(t-t_{r_{0}}-\sqrt{\frac{x_{c}}{\alpha}}\right) \Leftrightarrow x_{c}=\frac{\alpha\left(t-t_{r 0}\right)^{2}}{4} .
$$

From Eq. (14) we see that in this model, $x_{c}$ follows a uniformly accelerated motion. This is illustrated by Fig. 8, on which we observe the trajectories of 60 electrons, obtained from the code EUTERPE (a), or calculated using the previous model, i.e. assuming constant velocities and $v_{r}\left(t_{r}\right)=\alpha\left(t_{r}-t_{r 0}\right)(\mathrm{b})$. The strong resemblance between these two sets of curves shows that the model reproduces correctly the dynamics of Brunel electrons inside the plasma. In particular, we observe that the calculated function $x_{c}(t)$ fits almost perfectly the inner caustic of the two panels.

These trajectory crossings lead to a moving peak in the density of hot electrons. We clearly observe this peak as it travels across the overdense plasma, by plotting the density profile of hot electrons (Fig. 9). Within the density gradient, this peak has a full-width at halfmaximum of $\Delta x_{\text {peak }} \approx \lambda_{L} / 100$. The inset in Fig. 9 shows that, as expected, the velocity of the peak, $v_{p}=d x_{c} / d t$, increases linearly in time. Moreover, the straight line on this inset proves that $v_{p}$ can be accurately fitted by $v_{p}=\alpha\left(t-t_{r 0}\right) / 2$, with the values of $\alpha$ and $t_{r 0}$ determined from Fig. $7\left(\alpha=1.3 c / T_{L}\right)$.

Figure 10 presents the influence of the laser amplitude $a_{0}$ on the properties of this density peak, obtained from PIC simulations. We observe in the upper panel that the density of the peak increases linearly with $a_{0}$. Qualitatively, this is due to the fact that the number of electrons which have to be pulled out of the plasma to screen the laser field increases linearly with its amplitude. The lower panel of Fig. 10 shows that the influence of $a_{0}$ on the peak velocity $v_{p}$ is much weaker, a result that can be interpreted using our previous analytical description. Assuming that, like in Brunel's model, $v_{r} \propto a_{0}$ [38] (i.e. $\left.\alpha=\alpha_{0} a_{0}\right)$ leads to $v_{p}=\alpha_{0} a_{0}\left(t-t_{r 0}\right) / 2=\sqrt{\alpha_{0} a_{0} x_{c}}$. This theoretical fit is compared to numerical results in Fig. 10, showing that this simple formula provides a good approximation of the variation of $v_{p}$ with $a_{0}$.

All these results confirms that the model developed in this section is predictive, and accurately describes the trajectory crossing of Brunel electrons in the plasma, the resulting formation of an electron density peak as well as its propagation in the density gradient. We now study the collective oscillations of the plasma thermal electrons that are excited in the wake of this propagating density peak.

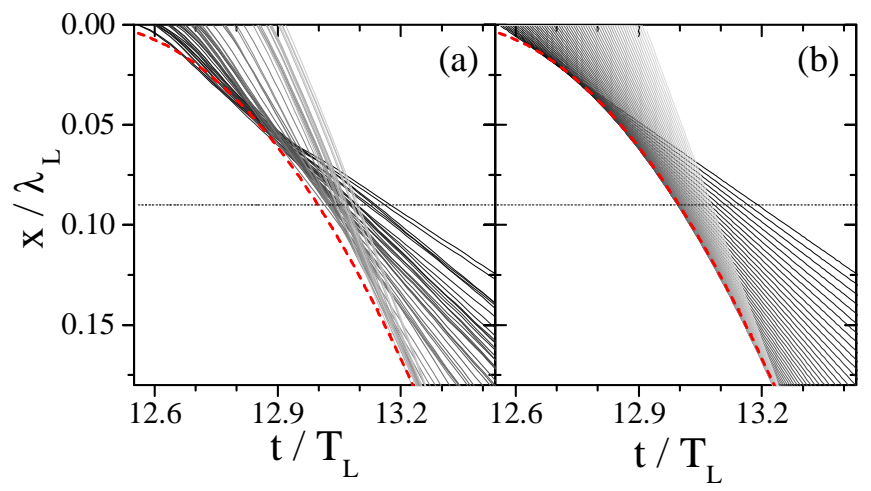

FIG. 8: Trajectories $x(t)$ of Brunel electrons traveling across the plasma. (a) PIC results. (b) Analytical trajectories. The origin $x=0$ corresponds to the position of the critical density surface, and the dotted line to the top of the density gradient, beyond which the plasma is homogeneous. The dashed line is a plot of the function $x_{c}(t)=\alpha\left(t-t_{r 0}\right)^{2} / 4$. 


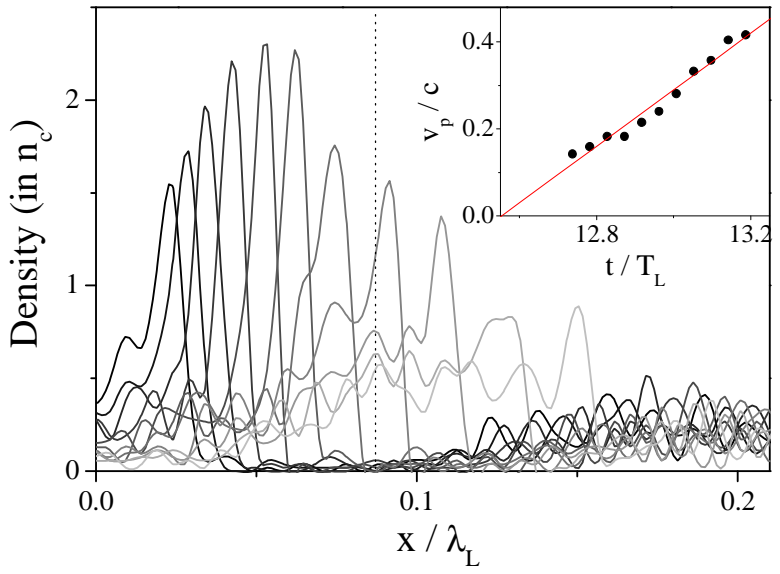

FIG. 9: Propagation of a peak of density in the overdense plasma. The main panel represents the density profile of electrons with velocities $v_{x}>0.1 c$, for 11 different times. The delay between two successive profiles is $0.045 T_{L}$. The dashed line indicates the position of the top of the density gradient. The inset shows the temporal evolution of the density peak velocity $v_{p}$.

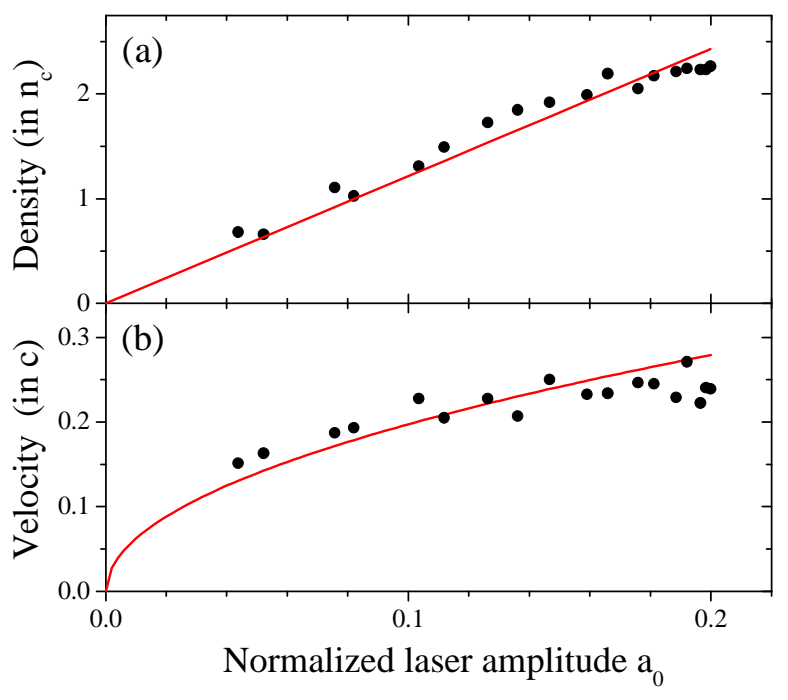

FIG. 10: Variations with the laser amplitude of the magnitude of the electron density peak (a), and of its velocity $v_{p}$ at $x=0.06 \lambda_{L}(\mathrm{~b})$. The lines are theoretical fits.

\section{Excitation of plasma oscillations}

\section{Analysis in the boosted frame}

In the boosted frame, the excitation of plasma oscillations by Brunel electrons can be analyzed using a simple 1D model. Here, we make two assumptions. First, we assume that the density peak resulting from the crossing of Brunel electrons acts as a point charge, of magnitude $-N e$ and velocity $v_{p}$. This is justified by the fact that this peak is significantly shorter than the plasma period: $\Delta x_{\text {peak }} / v_{p} \approx T_{L} / 30<2 \pi / \omega_{p}$, with $\omega_{p} \lesssim 15-30 \omega_{L}$ in a typical case. Note that when the plasma density gets very high, the density peak can become too long to excite efficiently plasma oscillations. This eventually limits the highest harmonic order that can be generated by CWE. For instance, in the case of Fig. 9, the highest frequency which can be excited is about $30 \omega_{L}$. Second, in order to reduce the problem to a single variable $\xi=x-v_{p} t$, we neglect the temporal variations of $v_{p}$ and consider a spatially-homogeneous plasma. In these conditions, the electron fluid equation of motion, and the continuity and Maxwell-Gauss equations, linearized for a non-relativistic cold electrons fluid are [49]:

$$
\begin{array}{r}
-v_{p} \partial_{\xi} v_{1}=-e E_{1} / m \\
-v_{p} \partial_{\xi} n_{1}+n_{0} \partial_{\xi} v_{1}=0 \\
\partial_{\xi} E_{1}=-\frac{e}{\epsilon_{0}}\left[n_{1}+N \delta(\xi)\right],
\end{array}
$$

where $E_{1}$ is the electric field along $\boldsymbol{e}_{x}, v_{1}$ the fluid velocity in the same direction and $n_{e}=n_{0}+n_{1}$ its total density, with $n_{0}$ the initial density and $n_{1} \ll n_{0}$ the induced density pertubation.

To solve for $n_{1}$, we combine Eq. (17) with the derivative of Eqs. (15) and (16) to obtain,

$$
\partial_{\xi}^{2} n_{1}+k_{p}^{2} n_{1}=-k_{p}^{2} N \delta(\xi)
$$

where $k_{p}=\omega_{p} / v_{p}$. The solution of this equation is the Green's function of a harmonic oscillator,

$$
n_{1}=\left\{\begin{array}{ll}
-N k_{p} \sin \left[k_{p} \xi\right] & \text { for } \xi<0 \\
0 & \text { for } \xi>0
\end{array} .\right.
$$

Then from Eqs. (15) and (16), we get the electric field behind the peak $(\xi<0)$,

$$
E_{1}=-\frac{N e}{\epsilon_{0}} \cos \left[k_{p} \xi\right]=-\frac{N e}{\epsilon_{0}} \cos \left[\omega_{p}\left(t-x / v_{p}\right)\right] .
$$

The moving density peak thus excites in its wake plasma oscillations of frequency $\omega_{p}$, with a delay $x / v_{p}$ due to its propagation.

The case of a density peak crossing an exponential density gradient, more relevant for CWE, is more complex. Nevertheless, if the gradient scale length $L$ is sufficiently large, we can assume that the plasma is locally homogeneous and describe the interaction using the previous formula, now with a space-dependent plasma frequency:

$$
E_{1}=\left(N e / \epsilon_{0}\right) \cos \left[\omega_{p}(x)\left(t-x / v_{p}\right)\right] .
$$

This is reasonable as long as $k_{p}=\omega_{p} / v_{p}$ is slowly varying over a plasma wavelength, $\lambda_{p}=2 \pi / k_{p} \ll$ $\left(d \ln k_{p} / d x\right)^{-1}=2 L$, leading to $L \gg \lambda_{L} / 80$ for $\omega_{p}=$ $10 \omega_{L}$ and $v_{p}=c / 4$. Since the typical scale lengths relevant to our study are about $\lambda_{L} / 10-\lambda_{L} / 100$ (see Sec. II A), we cannot use this approximation without further investigation.

To test this model, we performed PIC simulations (EUTERPE), without any laser field, but with a short bunch 


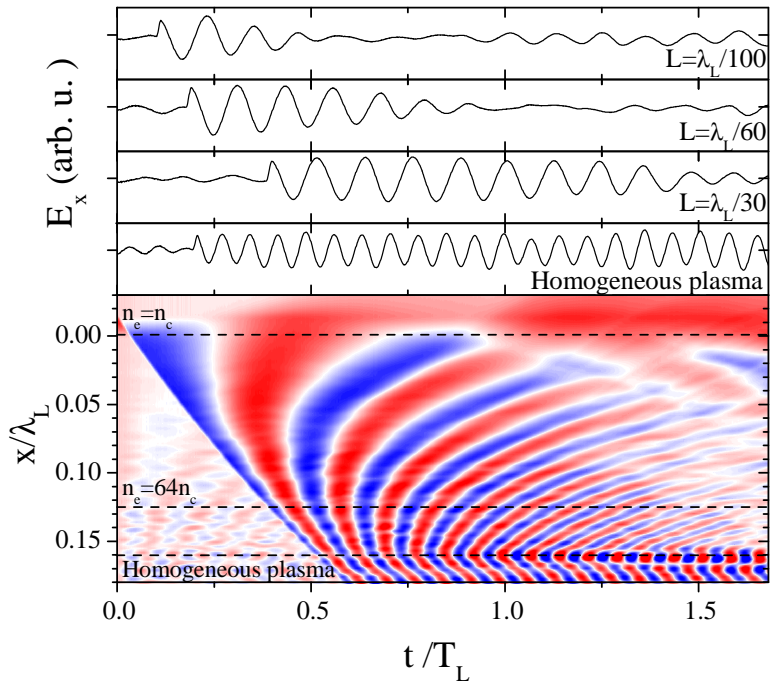

FIG. 11: (Color online) Excitation of plasma oscillations by an injected electron bunch, crossing an exponential density gradient followed by an homogeneous plasma. The lower panel shows the contour plot of the electric field $E_{x}$ for $L=\lambda_{L} / 30$. The top panels represent the profiles of $E_{x}$ around $n_{e}=64 n_{c}$, for different gradient scale length $L$ (three upper panels), as well as inside the homogeneous part of the plasma for the case $L=\lambda_{L} / 100$.

of electrons with a peak density of a few $n_{c}$, initially placed in vacuum, in front of a plasma with an exponential density gradient, and traveling toward this plasma with an initial velocity $v_{p}=c / 4$. The contour plot of Fig. 11 shows that for $L=\lambda_{L} / 30$, plasma oscillations are excited, all along the gradient, in the wake of this charge bunch. The wavefronts of the plasma oscillations are observed to progressively bend after the excitation. This bending is due to the spatial dependence of $\omega_{p}$ in the density gradient, and is satisfactorily reproduced by Eq. (21).

However, the observed damping of the collective oscillations in the density gradient are not predicted by this equation. This dumping effect, which gets stronger when $L$ decreases (see upper panels), is due to the spatial inhomogeneity of the plasma. Nevertheless, this effect is not crucial for the modeling of CWE (as long as $L \gtrsim \lambda_{L} / 100$ ), since, as we will see in Sec. IV D, only the first few cycles of the plasma oscillations following the excitation contribute to the harmonic emission.

Figure 12 shows the influence of the total charge of the exciting bunch on the amplitude of the triggered plasma oscillations, at a given point inside the density gradient. According to this figure, $E_{1}$ varies linearly with the density of the peak, which is in agreement with Eq. (20). Note that the oscillations do not exactly vanish when no electrons are sent into the plasma, because the plasma is initially not at equilibrium.

We now turn back to the study of CWE, where the density peak is formed by crossing of trajectories of laseraccelerated electrons. In Fig. 13, we have plotted the tra-

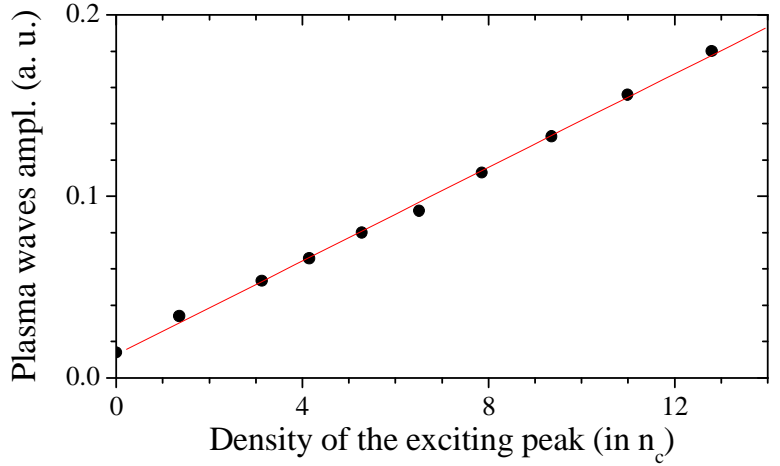

FIG. 12: Amplitude of the excited plasma waves around $100 n_{c}$, as a function of total charge of the injected electron bunch, for $L=\lambda_{L} / 60$.

jectories of a bunch of Brunel electrons which are crossing in the density gradient, and superimposed these curves on a contour plot of the electric field $E_{x}$. We observe that the density peak formed by the crossing of trajectories excites plasma oscillations, just as the bunch of electrons of Fig. 11. Moreover, Fig. 3 in [32] shows that these plasma oscillation wavefronts are well-reproduced by Eq. (20). This indicates that the physics is similar in the two cases.

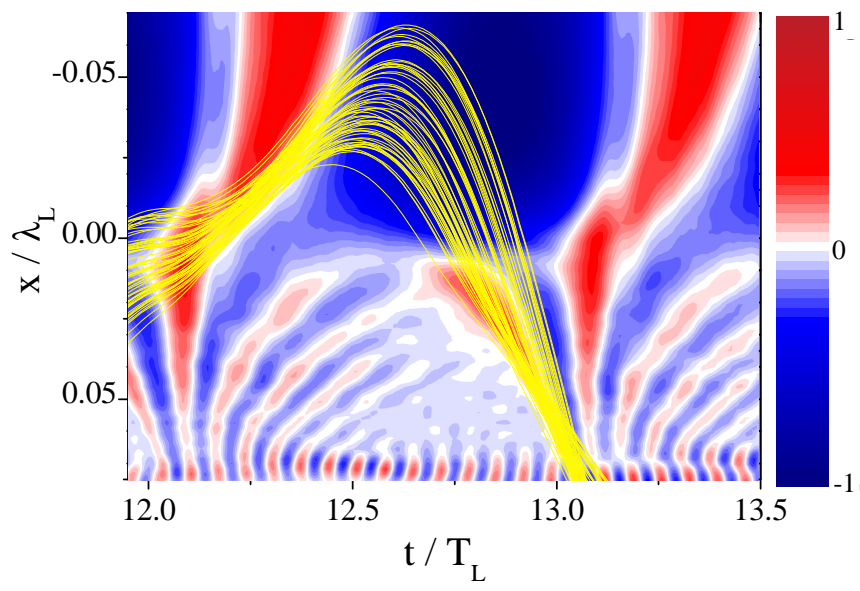

FIG. 13: (Color online) Excitation of plasma oscillations by Brunel electrons. The selected Brunel electrons, which trajectories are shown by the yellow lines, cross in the density gradient, and form a peak of electron density that travels through the plasma and excites in its wake plasma oscillations. These oscillations can be observed on the contour color map of $E_{x}$, on the right side of these trajectories. The oscillations observed at earlier time, are due to the Brunel electrons from the previous laser period (not shown). The simulation conditions are the same as for Figs. 7-9. 


\section{Analysis in the laboratory frame}

Although the analysis in the boosted frame is physically rigorous, it is instructive to also consider the excitation of plasma oscillations in the laboratory frame. Because the laser field has an oblique incidence in this frame, one should not consider a simple peak of density as in the previous 1D analysis, but rather a density front (or a density 'sheet' in 3D).

Indeed, for an incident plane wave, any perturbation induced by the laser at the point $(x=0, y=0)$ at $t=0$ reaches the point $(0, y)$ at $t=y \sin \theta / c$, where $\theta$ is the incidence angle. Hence, assuming a constant velocity $v_{p} \boldsymbol{e}_{x}$, a Brunel density peak originating from $(0, y)$ arrives at the point $(x, y)$ at the time $t_{0}=y \sin \theta / c+x / v_{p}$. Brunel electrons returning to the plasma thus form a density front, which sweeps along the target surface with the same phase velocity as the laser, $c / \sin \theta$. The angle between this front and the plasma surface is $\theta_{d f}=$ $\arctan \left(v_{p} \sin \theta / c\right)$ leading to $\theta_{d f} \approx 13^{\circ}$ for $v_{p}=c / 3$ and $\theta=45^{\circ}$.

Figure 14, which presents results from a 2D PIC simulation performed with the code CALDER, provides a direct confirmation of these predictions. Indeed, we observe that during each optical cycle of the laser, electrons with velocities $v_{x}>0.27 c$ form a density front which extends from the critical surface up to the homogeneous plasma, and excites plasma oscillations in its wake. From the mean angle $\theta_{d f} \approx 13.5^{\circ}$ measured in Fig. 14, we can infer that the mean velocity of the peak is about $0.34 c$ in this case.

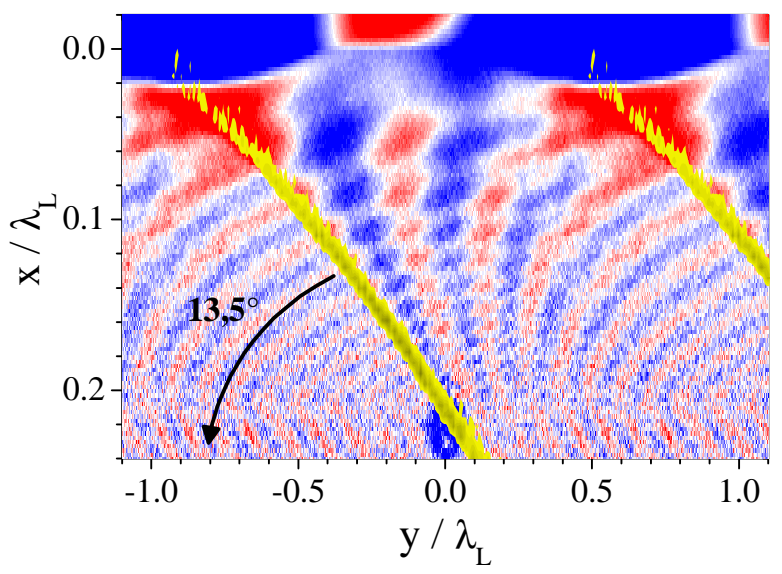

FIG. 14: (Color online) Excitation of plasma oscillations in the laboratory frame. The contour plot of $E_{x}(x, y)$, in a saturated blue/red color scale, reveals the plasma oscillations. The density of fast electrons $\left(v_{x}>0.27 c\right)$ is represented in a yellow color scale. These data were obtained from the PIC code CALDER for $a_{0}=0.2, L=\lambda_{L} / 20$ and $n_{e}^{\max }=225 n_{c}$.

Like in the boosted frame, this density front is observed to trigger plasma oscillations. To describe this excitation analytically, we generalize the model of the previous section, by simply setting $\xi=x-v_{p}(t-y \sin \theta / c)$. This leads to

$$
\begin{aligned}
E_{1} & =-\left(N e / \epsilon_{0}\right) \cos \left[\omega_{p}\left(t-x / v_{p}-y \sin \theta / c\right)\right] \\
& =-\left(N e / \epsilon_{0}\right) \cos \phi(x, y, t) .
\end{aligned}
$$

This equation satisfactorily reproduces the wavefronts of Fig. 14.

\section{Emission of attosecond XUV pulses}

\section{Observation in PIC simulations}

We now study the final step of the CWE mechanism, which is the emission of light by the excited plasma oscillations. Such an emission can only occur in an inhomogeneous plasma. Indeed, in a homogeneous plasma, the wavefronts of the electron plasma oscillations are parallel to the triggering density front. Since the excited electrons oscillate perpendicularly to this front, the electron oscillations in such a plasma are purely longitudinal $\left(\boldsymbol{j} \times \boldsymbol{k}^{\mathrm{PO}}=0\right.$, where $\boldsymbol{k}^{\mathrm{PO}}$ is the wave vector of the plasma oscillations). They can therefore not emit light (see Sec. III D).

The situation is quite different in the density gradient at the plasma mirror surface. Indeed, Fig. 14 shows that in this case, the wavefronts of the plasma oscillations curve in time. Assuming that electrons keep their initial direction of oscillation (as verified in Ref. [50]), i.e. perpendicular to the exciting density front, the plasma oscillations which are initially purely longitudinal, progressively acquire a transverse component, and can therefore radiate an electromagnetic wave.

Light emission by plasma oscillations is thus only expected in the density gradient. This is indeed what is observed in Fig. 15(a), which shows the results of a 2D CALDER simulation. Soon after the excitation of plasma oscillations by the Brunel density front, the emission of an attosecond pulse is observed. Such a pulse is emitted during each optical cycle of the incident laser, resulting in a train of attosecond pulses, associated in the spectral domain to high order harmonics of the laser.

These harmonics are observed on the spatially resolved spectrum of Fig. 15(b), which indicates that the harmonic of order $n$ comes from the area of the density gradient where $\omega_{p}(x)=n \omega_{L}$. This shows that CWE emission is due to a distribution of locally harmonic currents, with a spatially-varying local frequency. Since the maximum frequency reached by this current distribution is the maximum plasma frequency $\omega_{p}^{\max }$ of the target, the harmonic spectrum presents a sharp cut-off at $\omega_{p}^{\max }$. This important feature of CWE clearly appears in Fig. 15(b), and has been verified experimentally in 2007, by focusing an intense femtosecond laser on materials of different densities (see Refs. [51, 52] and Sec. VIB 1).

An essential feature of Fig. 15(a) is the fact that light emission by plasma oscillations is confined in time, to a fraction of the laser optical cycle, leading to the emission 
of attosecond pulses. Although this effect could in principle be attributed to the damping of plasma oscillations in the density gradient, described in Sec. IV C, this damping is too slow to provide a quantitative interpretation, especially for the gradient scale length used in Fig. 15.

In references [32, 53, 54], this temporally-confined emission is interpreted using phase matching arguments. In these articles, the emission at $\omega_{p}(x)$ is actually considered to occur when $\boldsymbol{k}^{P O}$ matches the wave vector, around its turning point, of an electromagnetic wave of frequency $\omega=\omega_{p}(x)$, emerging from the density gradient with an angle $\theta$. This requires that $k_{x}^{P O}=0$, in other words, the emission is expected to be confined around the time when the wavefronts of the plasma oscillations are perpendicular to the plasma surface. This is indeed what is qualitatively observed on Fig. 15(a).

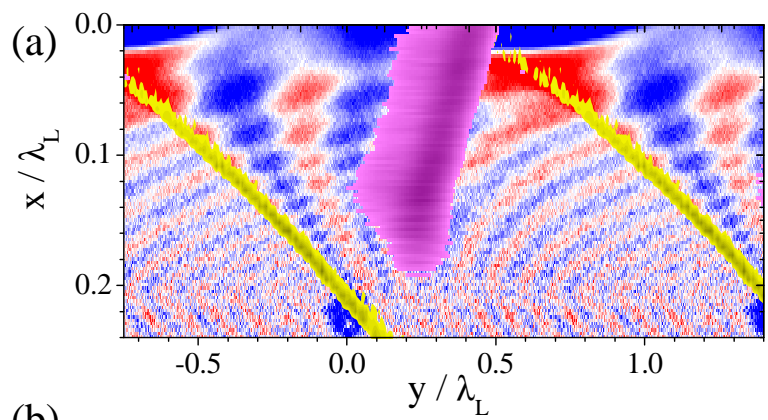

(b)

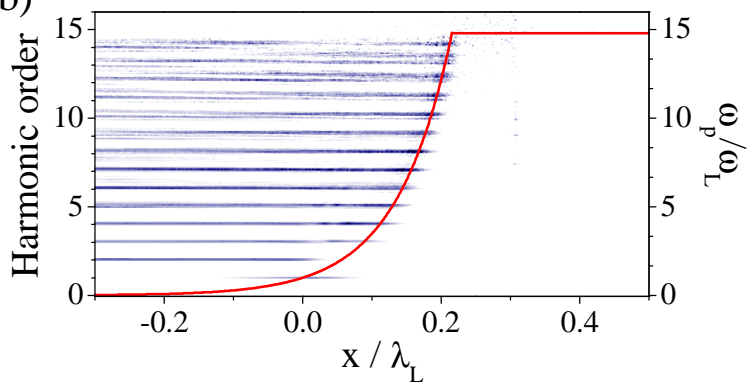

FIG. 15: (Color online) Emission of attosecond pulses and high order harmonics. (a) Contour plot of the $B_{z}$ field, filtered between $4 \omega_{L}$ and $14 \omega_{L}$ (in purple), superposed on the contour plots of Fig. 14. (b) Spatially resolved emission spectrum (contour plot) and plasma frequency profile (straight line). A smooth spectral correction has been applied to balance the harmonic amplitudes and make them all clearly visible simultaneously on a linear scale. These results come from a CALDER simulation performed for $a_{0}=0.2, n_{e}^{\max }=225 n_{c}$ and $L=\lambda_{L} / 25$.

Here, we provide a more detailed -although not yet totally rigorous- analysis of the conditions required for the emission to occur, by considering the transverse current $j_{t}(x, t) \boldsymbol{e}_{y}$ associated to plasma oscillations in the boosted frame, and by calculating the field radiated by this current as described in Sec. III.

\section{Analysis using the transverse current distribution}

At first order, the current associated to plasma oscillations in the boosted frame is the obliquity current $j_{t}=j_{y}(x, t)=e n_{1} c \sin \theta$. Using Eq. (19) for $n_{1}$ leads to

$$
j_{t}(x, t)=-\omega_{p}(x) \frac{N e c \sin \theta}{v_{p}} \sin \phi(x, t)
$$

with $\phi(x, t)=\omega_{p}(x)\left(t-x / v_{p}\right)$.

Equation (23) shows that the transverse current is non-zero in the boosted frame (as opposed to the laboratory frame) as soon as $n_{1} \neq 0$. But this does not guarantee that light emission is efficient, as the emitted field is an integral of $j_{t}$ (see Eq. (6)). According to Eq. (5), the spectrum of the emission in vacuum is given by $S(\omega)=\mu_{0}^{2}\left|\hat{\hat{j}}_{t}(k=\omega / c, \omega)\right|^{2}$. The function $\left|\hat{\hat{j}}_{t}(k, \omega)\right|^{2}$ is plotted in Fig. 16, for two different current distributions (right panels) calculated using Eq. (23) for two consecutive laser optical periods. We consider first the case of Fig. 16(a), where the exciting electron density peak travels through the density gradient from the vacuum up to the homogeneous plasma, which corresponds to the typical CWE case.

To understand the shape of this 2D Fourier-transform, let us consider the local wave vector $k_{x}^{\mathrm{PO}}(x, t)=-\partial \phi / \partial x$ of the electron oscillations, given in the case of an exponential density gradient by:

$$
k_{x}^{\mathrm{PO}}=\frac{\omega_{p}(x)}{c}\left(\frac{c\left(t-x / v_{p}\right)}{2 L}-\frac{c}{v_{p}}\right) .
$$

This expression shows that $k_{x}^{\mathrm{PO}}$ increases in time, from $k_{x}^{i}=-\omega_{p}(x) / v_{p}$ at the time $t=x / v_{p}$ when the plasma oscillation is excited at point $x$, up to $k_{x}^{f}=$ $\omega_{p}(x) / c\left(\lambda_{L} / 2 L-c / v_{p}\right)$ at $t=x / v_{p}+T_{L}$, the time at which a new peak of density crosses the gradient and excites a new set of plasma oscillations. The wavevector $k_{x}^{\mathrm{PO}}$ sweeps across this interval as time evolve within a laser optical period. This temporal evolution of the local wavevector in direct space qualitatively explains the shape of $\hat{\hat{j}}_{t}$ in reciprocal space: this function is bounded between the two limiting lines $k_{x}^{i}$ and $k_{x}^{f}$, leading to the $\mathrm{V}$ shape observed in the left panel of Fig. 16(a).

Since the emitted spectrum is given by a lineout of $\left|\hat{\hat{j}}_{t}\right|^{2}$ along the line $k=\omega / c$, a significant light emission occurs only if this line is included in this cone. This occurs if

$$
k_{x}^{f} \geq \omega / c \Longleftrightarrow 2 L \leq \lambda_{L} v_{p} /\left(c+v_{p}\right) .
$$

For $v_{p}=c / 4$, light emission is thus efficient for all $L \leq$ $\lambda_{L} / 10$. For longer gradient, the temporal evolution of $k_{x}^{\mathrm{PO}}$ is too slow, so that it fails to reach $\omega / c$ within one laser optical cycle.

Assuming a one-to-one mapping between the local wavevector in direct space $k_{x}^{\mathrm{PO}}$ and the wavevector in reciprocal space, Eq. (24) provides an estimation of the emission time $t_{e}$ of harmonic $n_{H}$ (with respect to time 
$t=0$ where the exciting bunch crosses $x=0$, where $\left.n=n_{c}\right)$, for which $k_{x}^{\mathrm{PO}}\left(t_{e}\right)=n_{H} \omega_{L} / c$ at $x_{H}=2 L \ln n_{H}$ :

$$
t_{e}=\frac{2 L}{c}\left[1+\frac{c}{v_{p}}\left(1+\ln n_{H}\right)\right] .
$$

It is instructive to also consider the case where the exciting density front goes from the denser part of the plasma toward the vacuum (case of Fig. 16(b)). This typically corresponds to what occurs at the rear-side of laser-irradiated thin foils $[30,31]$. In this case, $k_{x}$ decreases in time, starting from $k_{x}^{i}=-\omega_{p}(x) / v_{p}$. As a result, the line $k=\omega / c$ does not intersect the intense part of $\hat{\hat{j}}_{t}^{2}$, because this distribution is not located in the right quadrant of the $(\omega, k)$ space. In this case, plasma oscillations cannot emit electromagnetic waves, even for the shortest gradient scale lengths. This is the reason why plasma oscillations excited at the rear surface of thin targets in the wake of outgoing Brunel density peaks can not radiate light, as demonstrated in Ref. [55].

We emphasize that this analysis leads to the same conclusion as the qualitative discussion of Sec. IV D 1, based on phase matching, i.e. that the generation of high order harmonics is possible only when plasma oscillations of increasing frequencies are excited with an increasing delay. These two descriptions however lead to slightly different emission conditions on $k_{x}^{P O}$, and hence to slightly different times of emission. Using Eq. (24) leads to a difference of $2 L / c$ between these two times, i.e. $T_{L} / 12.5$ in the case of Fig. 15 -shorter than the duration of the emitted attosecond pulse.

This analysis of the emission process is still incomplete. Indeed, the expression used for the transverse current totally neglects the polarization induced in the density gradient by the generated electromagnetic field, as it propagates from the emission point to vacuum. In other words, it omits the refraction of the harmonic field by the plasma. The spectrum intersecting the line $k=\omega / c$ on Fig. 16 thus corresponds to the one that would be obtained if the emitted electromagnetic waves were traveling only in a vacuum. Such an approximation is quite crude, as each harmonic is emitted in an area where the plasma is overdense precisely at this frequency. These effects are likely to affect the exact shape of the emitted spectrum $S(\omega)$, but the general conclusions of the previous analysis should remain valid.

To go further and evaluate the actual generation efficiency, an alternative is to use the linear mode conversion theory already developed to study direct and inverse resonant absorption $[37,56,57]$, in the simpler case where plasma oscillations are excited at a single frequency $\omega$ in the density gradient. These models describe the coupling between plasma and electromagnetic waves in a gradient with a linear density profile, in the case of an unmagnetized collisionless plasma. Hinkel-Lipsker et al. demonstrated that the conversion efficiency $\eta$ is a function of $m=\left(\omega L_{\omega} / c\right)^{2 / 3} \sin ^{2} \theta$, with $L_{\omega}=\left(d \ln n_{e} / d x\right)^{-1}$, which admits a maximum for $m \approx 0.5$ [57]. For the parametric
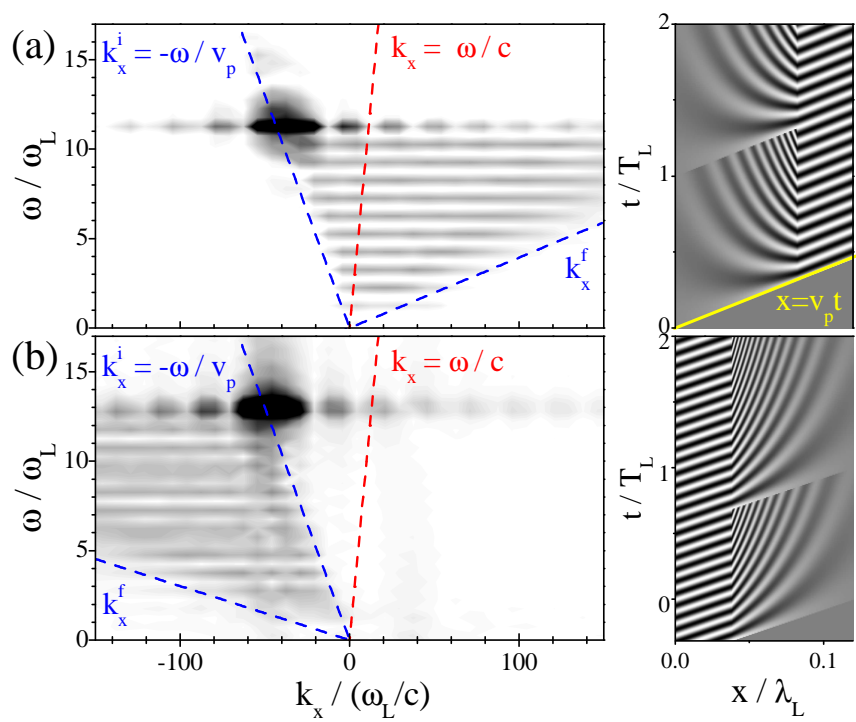

FIG. 16: (Color online) Fourier transforms $\left|\hat{\hat{j}}_{t}(k, \omega)\right|^{2}$ of the transverse current in the boosted frame, with respect to both time and space. The left panels are Fourier transforms of the currents plotted on the right panels. (a) Case of an exciting density front going from a low density to a high density region of the plasma $\left(\omega_{p}^{\max }=11 \omega_{L}\right)$. (b) Opposite case. On both panels, $L=\lambda_{L} / 60$ and $v_{p}=0.26 c$. The blue dashed lines show the boundaries $k_{x}^{i}(\omega)$ and $k_{x}^{f}(\omega)$, and the red dashed line the $\omega=k / c$ lineout which gives the spectrum of the emitted light.

study of the next session, we will use the approximate expression of $\eta$ established in [58]:

$$
\eta=2 \alpha m(2+\alpha m)^{-1} \exp \left(-4 m^{3 / 2} / 3\right),
$$

where $\alpha=2.644$.

Remark We note that CWE is sometimes confused with earlier models developed in the 80's [20, 21] to interpret some of the first experiments on HHG on solid targets $[18,19]$, which suggested harmonic frequency cutoffs around $\omega_{p}^{\max }$. In these models, the coupling of the laser to resonant plasma oscillations leads to the production of its second harmonic. Then, harmonic $n+1$ is produced by the coupling between harmonic $n$ and resonant plasma oscillations of higher frequencies. HHG results from this cascaded linear mode conversion process in the plasma. This is very different from CWE, where all plasma oscillations are excited by energetic electrons traveling across the density gradient [59].

\section{E. Parametric study}

Sections IV A to IV D provide a complete description of CWE, but mostly for fixed physical parameters. We now present a parametric study of this process, and show how the laser intensity, the density gradient and the incidence angle influence the generation mechanism. 


\section{Laser intensity}

We first examine the effect of laser intensity on the harmonic signal. We have demonstrated in Sec. IV B that the amplitude of the exciting density front grows linearly with the laser field amplitude, and in Sec. IV C that the amplitude of the excited plasma oscillations is proportional to this density. Since the conversion from plasma oscillations to light is also a linear mechanism, the CWE signal is thus expected to vary linearly with laser intensity, i.e. to have a constant generation efficiency. This prediction is supported by the PIC simulation results displayed in Fig. 17. In this intensity range, Coherent Wake Emission is thus a linear mechanism of high-order harmonic generation.

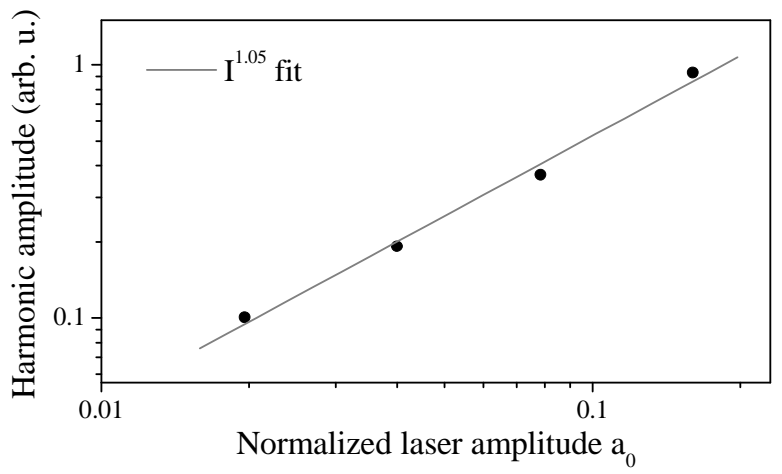

FIG. 17: Influence of the laser field amplitude. Each point corresponds to the amplitude of the harmonic signal, integrated between orders 4 to 10 . These results were obtained with the 2D3V code CALDER for $L=\lambda_{L} / 30$ and $n_{e}^{\max }=110 n_{c}$.

This linear behavior can however not be extrapolated to arbitrary low laser intensities. As $a_{0}$ goes down, Brunel electrons cross nearer and nearer from the critical surface (according to Eq. 14, $x_{c}(t)=\alpha_{0} a_{0}\left(t-t_{r 0}\right)^{2} / 4$ with $\left.t \leq t_{m}\right)$. When $x_{c}\left(t_{m}\right)$ eventually becomes smaller than the distance between the critical density and the point where the second harmonic is generated, Brunel electrons can no more excite efficiently plasma oscillations at laser harmonic frequencies, and the CWE signal vanishes. If we assume that $t_{m}-t_{r 0} \approx 0.6 T_{L}$ (see Secs. IV A and IV B), and use the value of $\alpha_{0}$ obtained from PIC simulations in Sec. IVB $\left(\alpha_{0}=\right.$ $\left.6.5 c / T_{L}\right)$, we find that the harmonic emission occurs only if $a_{0}>8 \ln 2 L \alpha_{0}^{-1}\left(t_{m}-t_{r 0}\right)^{-2} \approx 3.4 L / \lambda_{L}$, that is $I \lambda^{2} \gtrsim 2 \times 10^{15} \mathrm{~W} \mathrm{~cm}^{-2} \mu \mathrm{m}^{2}$ for $L=\lambda_{L} / 100$. This is in remarkable agreement with experimental observations (see Sec. VI C 1)

\section{Density gradient}

a. Influence of the gradient scale length. We now study the influence of the scale length $L$ of the exponential density gradient on the generation efficiency. Two simulation spectra obtained for two different scale lengths are plotted in Fig. 18(a). The strong differences between these two curves reveal that the gradient scale length plays a key role in CWE.

To explain this observation, we consider Fig. 18(b), where the generation efficiencies of harmonics 3 (H3) and 8 (H8) have been plotted as a function of $L$. We first note that no harmonic is produced when $L=0$. This is in agreement with Sec. IV D where we demonstrated that CWE only occurs inside the inhomogeneous plasma formed by the density gradient. In addition, we observe that the harmonic signal first increases steeply with $L$, before decreasing gently for 'long' gradients. This indicates the existence of an optimum scale length [60]. This optimum length decreases with harmonic order.
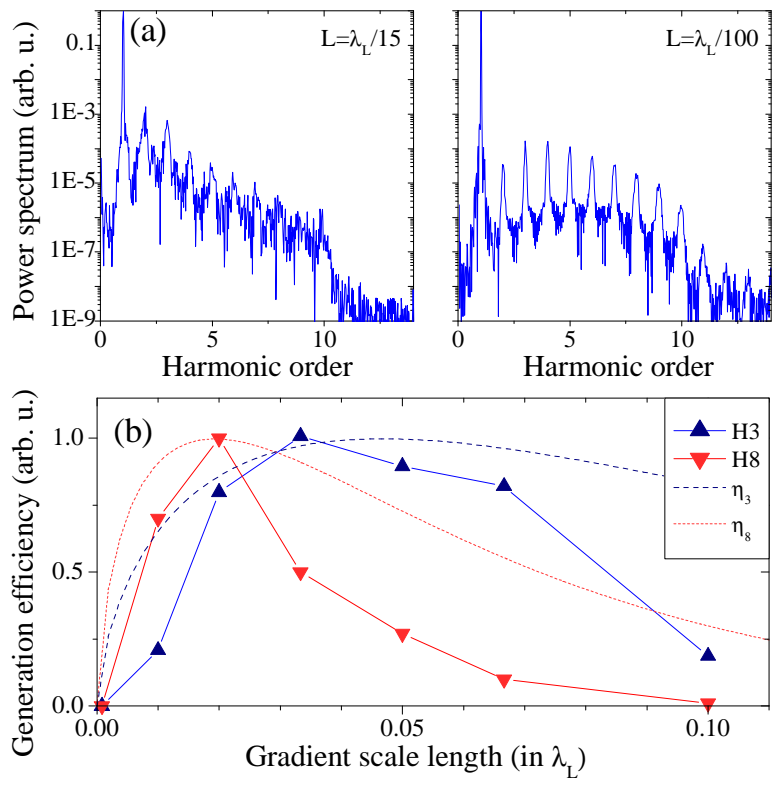

FIG. 18: (Color online) Influence of the gradient scale length. (a) Spectra obtained for $L=\lambda_{L} / 15$ and $L=\lambda_{L} / 100$. (b) Variation with the scale length of the generation efficiency of harmonics 3 and 8 . The dashed lines correspond to the function $\eta$ calculated for each harmonic using Eq. (27). Simulations were performed with the code CALDER for $a_{0}=0.2$ and $n_{e}^{\max }=110 n_{c}$.

To interpret these results, we analyze first the variation with $L$ of the conversion efficiency $\eta$ of plasma oscillations into light. To this end, we consider the exponential gradient as locally linear, and use Eq. (27) to estimate $\eta(x)$. The two theoretical curves thus obtained for harmonics 3 and 8 are plotted in Fig. 18(b). Although these curves reproduce the general behavior of the PIC results, their variations are much gentler.

The reason for this discrepancy is that the amplitude $N$ of the electron density peak, which excites plasma oscillations, also varies with $L$. Figure 9 indeed shows that $N$ depends on the distance $x$ to the plasma critical density surface. When $L$ is increased, the function $N(x)$ hardly changes, but the point $x_{H}=2 L \ln n_{H}$, where har- 
monic order $n_{H}$ is produced, moves away from the critical surface. As a result, the amplitude of the emitting plasma oscillations, which is proportional to $N\left(x_{H}\right)$, varies with $L$, and so does the overall generation efficiency.

Our model is too simple to quantitatively predict the shape of $N(x)$. We can, however, roughly estimate its variations. According to Fig. 9, the maximum of $N(x)$ is reached at $x_{\max } \approx 0.065 \lambda_{L}$. The optimum gradient scale length is thus of the order of $\lambda_{L} / 30$ for the third harmonic and $\lambda_{L} / 60$ for the $8^{t h}$. These values correspond approximately to the positions of the maxima found in Fig. 18. In addition, we observe in Fig. 9 that $N(x)$ tends to zero for $x \approx 0.2 \lambda_{L}$. A strong reduction of the harmonic 3 (resp. 8) signal when $L$ tends to $\lambda_{L} / 10\left(\right.$ resp. $\left.\lambda_{L} / 20\right)$ can therefore be anticipated, and is indeed observed in Fig. 18 .

b. Influence of the shape of the density gradient. Experimentally, density gradients are generally not perfectly exponential, and recent results indicate that their shape can actually strongly affects CWE harmonic spectra [61]. This is confirmed by a comparison of the simulated spectra of Figs. 19(a) and 19(b) which correspond respectively to an exponential and a linear density gradient. We observe that fewer harmonics are efficiently generated in the case of a linear gradient.

This is partly explained by the fact that the linear conversion efficiency $\eta$ varies much more strongly with harmonic order in this case. This clearly appears on Fig. 19(d), which shows that a linear gradient acts as a band-pass filter, the central frequency of which depends on the slope of the density gradient.
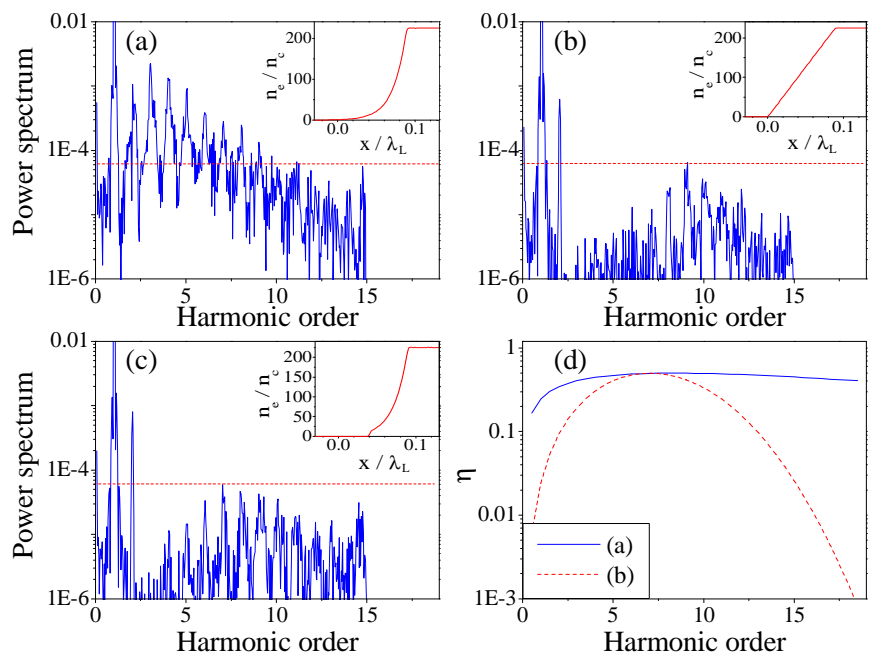

FIG. 19: (Color online) Harmonic spectra for different density gradients. (a) Exponential density gradient with $L=\lambda_{L} / 60$. (b) Linear gradient, $n_{e}(x)=225 n_{c} x / L$ with $L=\lambda_{L} / 11$. (c) Same exponential gradient as in (a), now truncated at $n_{e}(x)=13 n_{c}$ (see inset). These results come from EUTERPE simulations performed for $a_{0}=0.2$. (d) Linear conversion efficiency $\eta$ calculated with Eq. (27) for the gradients of (a) and (b), using $L_{\omega}=\left(\omega / \omega_{p}^{\max }\right)^{2} L$ in this equation for the linear density gradient, and $L_{\omega}=L$ for the exponential gradient.
The variation of $\eta$ are however not sufficient to totally explain the influence of the gradient shape. This is illustrated in Fig. 19(c) which displays a spectrum obtained for the same exponential gradient as before, now truncated at $n_{e}(x)=13 n_{c}$. In this case, the conversion efficiency $\eta$ is the same as in (a) for harmonic orders $n_{H} \geq 4$. The strong differences between the spectra of (a) and (c) for $4 \leq n_{H} \leq 8$ can thus not be attributed to $\eta$.

This type of effects can be interpreted by taking into account the additional influence of $N(x)$. The point $x_{\max }$ where $N(x)$ is maximal actually corresponds in (a) to the position where the third harmonic is generated, while in (c) it corresponds to the generation point of harmonic 9 . These values are in perfect agreement with the maxima of the spectra (a) and (c), suggesting that $N(x)$ is responsible for their differences. In (b), $N(x)$ is also maximal for harmonic 9 , and this explains why this spectrum has a shape similar to the one of Fig. 19(c). These two spectra however differ by the fact that more harmonics are efficiently generated with the truncated exponential, which is due to the variations of $\eta$.

In summary, the dependence of the harmonic generation efficiency on both the shape and the characteristic length of the density gradient can be interpreted semiquantitatively by the influence of $\eta$ and $N(x)$.

\section{Incidence angle}

To conclude this parametric study, we investigate the influence of the incident angle $\theta$ on the harmonic signal, for a fixed laser power (i.e. a laser intensity that is proportional to $\cos \theta$, like in an actual experiment). Figure 20 shows that the generation efficiency is maximum for $\theta \approx 55^{\circ}$ and tends to 0 when $\theta$ tends to $0^{\circ}$ (where the Brunel mechanisms no longer occurs) or $90^{\circ}$ (where the laser intensity tends to 0 ). It also shows that the optimum angle is slightly higher for the lower harmonics.

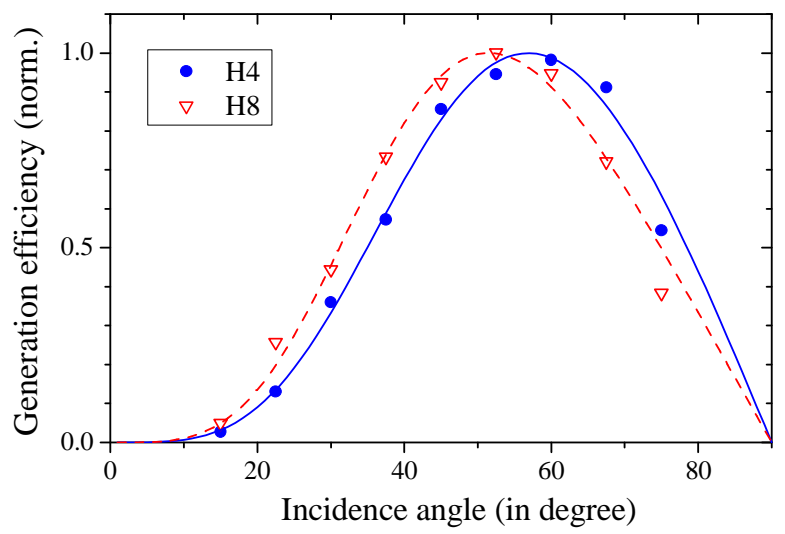

FIG. 20: (Color online) Variation with the incidence angle of the generation efficiency of harmonics 4 and 8 at a fixed incident laser power. The points are provided by EUTERPE simulations performed for $L=\lambda_{L} / 50$, and $a_{0}^{2}=0.08 \cos \theta$. The straight lines correspond to theoretical fits. 
These results can be interpreted by using the results of Sec. IV E 2, which demonstrate that the power generation efficiency of CWE varies as $\eta N^{2}$. The $\eta$ parameter is again provided by Eq. (27), and is a function of $m=\left(2 \pi n_{H} L / \lambda_{L}\right)^{2 / 3} \sin ^{2} \theta$. This immediately shows that the optimum angle should increase when $n_{H}$ decreases, as observed in Fig. 20. The variation of $N$ with $\theta$ can also be evaluated, in a very simple way: at fixed laser power, the laser intensity varies as $\cos \theta$ and the electric field $E_{0}$ as $\sqrt{\cos \theta}$. Further, the density of Brunel electrons depends only on the component of the electric field along the plasma normal, $E_{x}=E_{0} \sin \theta \propto \sin \theta \sqrt{\cos \theta}$ (see Sec. IV A). Since $N$ varies linearly with $E_{x}$, as shown by Fig. 10(a), we finally get $N^{2} \propto \sin ^{2} \theta \cos \theta$.

Taking into account the variations of both $\eta$ and $N^{2}$ for $n_{H}=4$ and $n_{H}=10$ leads the two theoretical curves plotted in Fig. 20. The agreement with the numerical points is remarkably good, confirming that despite its simplicity, our model provides a rather accurate description of the CWE mechanism.

\section{F. Phase properties}

In the previous sections, we have analyzed the influence of different physical parameters on the amplitude of the emitted harmonic field, but we have so far ignored their influence on the phase properties of this field. Studying these phase properties is however crucial, as they largely determine some of the main features of the XUV light source, such as the divergence of the beam, the duration of the attosecond pulses, or the spectral width of individual harmonics.

Many phenomena can, in principle, affect the phase properties of the generated field. As demonstrated in Ref. [62, 63], the main source of non-trivial phase is the variation of the emission time of the harmonics with both harmonic order and laser intensity. We first provide a simple approximate expression for this emission time, and then analyze the consequences on the phase properties of the source.

\section{Emission time.}

According to Eqs. (13) and (26), the frequency component $n_{H} \omega_{L}=\omega_{p}\left(x_{H}\right)$ emitted around $x_{H}$ crosses the critical surface of the plasma and escapes into vacuum at $\tau_{e}=t_{r}+t_{e}+t_{c}$, where

$$
t_{r}=t_{r 0}+\sqrt{x_{H} / \alpha_{0} a_{0}},
$$

is the return time at $x=0$ (where $n_{e}=n_{c}$ ) of the Brunel electrons that trigger plasma oscillations at $x_{H}$, and

$$
t_{e}=\frac{2 L}{c}+\frac{L}{v_{p}}\left(2+\frac{x_{H}}{L}\right)=\frac{2 L}{c}+\sqrt{\frac{x_{H}}{\alpha_{0} a_{0}}}\left(1+\frac{2 L}{x_{H}}\right),
$$

is the approximate delay required for these electrons to reach $x_{H}$, and for the light emission by plasma oscillations to occur at this point. This expression is obtained by combining Eq. (26) and the expression $v_{p}=\sqrt{\alpha_{0} a_{0} x_{c}}$ established in Sec. IV B. Finally,

$$
t_{c}=x_{H} / c
$$

is the time taken by the light to reach the critical surface, neglecting the plasma dispersion for simplicity. Summing Eqs. (28), (29) and (30), we get

$$
\tau_{e}=t_{r 0}+\frac{2 L+x_{H}}{c}+2 \sqrt{\frac{x_{H}}{\alpha_{0} a_{0}}}\left(1+\frac{L}{x_{H}}\right) .
$$

This equation shows that $\tau_{e}$ depends on the harmonic order $n_{H}=e^{x_{H} / 2 L}$, on the laser intensity $a_{0}$, and on the gradient scale length $L$. These different dependencies lead to various effects on the emitted field.

\section{Attosecond chirp.}

We first examine the consequences of the variation of $\tau_{e}$ with $n_{H}$. According to Eq. (31), we have

$$
\frac{\partial \tau_{e}}{\partial n_{H}}=\frac{1}{n_{H}}\left[\frac{2 L}{c}+\sqrt{\frac{2 L}{\alpha_{0} a_{0} \ln n_{H}}}\left(1-\frac{1}{2 \ln n_{H}}\right)\right] .
$$

The emission time is thus a growing function of $n_{H}$ for all $n_{H} \geq 2$. This means that high order harmonics emerge later from the plasma than low order harmonics. Physically, this comes from the fact that because higher harmonic orders are generated at the top of the density gradient (see Fig. 15(b)), they are excited at later time by Brunel electrons, and need more time to escape the plasma. As a result, CWE attosecond pulses, resulting from the superposition of a group of harmonics, are positively chirped. Because of this chirp, CWE attosecond pulses are significantly longer than the Fourier limit duration (Fig. 21), as suggested by the experimental results of Ref. [33].

Equation (32) does not only provide the sign of the chirp, it also indicates that it depends on $L$ and $a_{0}$. An increase of $L$, for instance, stretches the spatial distribution of harmonic sources, and hence increases the delay between the emission time of these different frequencies. The amplitude of the chirp, and hence the duration of the attosecond pulses, therefore grow with $L$, as confirmed by Fig. 21.

\section{Femtosecond chirp and spatial phase.}

According to Eq. (31), $\tau_{e}$ also depends on $a_{0}$ and $L$. These two quantities vary temporally during the interaction, leading to deviations from perfect periodicity of the CWE attosecond pulse train, and hence to a broadening 


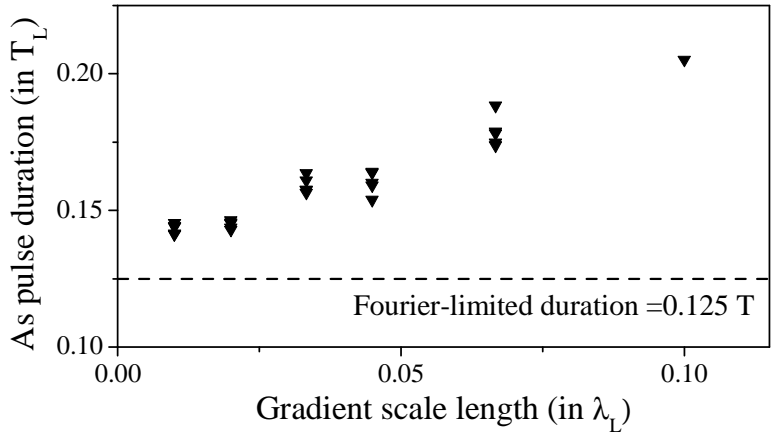

FIG. 21: Duration of CWE attosecond pulses for different gradient scale lengths, obtained from CALDER simulations. Different points for a given length are associated to different pulses in the attosecond train. These attosecond pulses correspond to the superposition of harmonics 4 to 10 . The simulation parameters are the same as for Fig. 18.

and a non-trivial spectral phase of the associated harmonics [32,62]. The normalized amplitude $a_{0}$ also varies spatially because of the intensity distribution of the focused laser beam. This intensity distribution can in turn lead to a spatial dependence of $L$. These two effects result in a spatial dependence of $\tau_{e}$, and, in analogy to the spectral domain, increase the divergence and distort the spatial phase of the harmonic beam [52, 62, 64].

According to Eq. (31), $\tau_{e}$ varies linearly with $L$, while it varies as $1 / \sqrt{a_{0}}$. Since $a_{0}$ goes from 0 to $a_{0}^{\max }$ during the laser pulse, the dominant contribution to variations of $\tau_{e}$ comes from its dependence on $a_{0}$, especially on the edges of the laser pulse where $a_{0} \rightarrow 0$. The dependence on $L$ only leads to small corrections, as demonstrated in [62]. In addition, Fig. 1 in [62] shows that neither the carrier envelope phase nor the chirp of the pulse varies significantly along the train.

The effect that primarily determines the temporal and spatial phase properties of individual CWE harmonics is thus the temporal shift, with hardly any distortion, of the emitted attosecond pulses when the laser field amplitude $a_{0}$ varies. This effect is illustrated in Fig. 22, which displays the delay between the emission of two successive attosecond pulses, $T_{i}(t)=\tau_{e}\left(t+T_{L}\right)-\tau_{e}(t)$, as a function of time during an incident laser pulse with a $\sin ^{2}$ intensity profile. The instantaneous frequency of the harmonics associated to this attosecond train varies as $1 / T_{i}$, so that these harmonics are chirped. This chirp is almost linear in the central portion of the pulse, but becomes stronger and more complex in the edges, as expected from the $1 / \sqrt{a_{0}}$ dependence of $\tau_{e}$.

A remarkable feature of the results of Fig. 22 is that the variations of $T_{i}$ are almost independent of the peak laser amplitude $a_{0}^{\max }$. In other word, $T_{i}$ depends on the laser pulse envelop but not on $a_{0}^{\max }$. This is confirmed by Fig. 23(b), where $\tau_{e}$ is observed to decrease by a constant amount when the peak intensity is reduced. This numerical finding, which is supported by experimental results (see Sec. VI), is in contradiction with the predictions of
Eq. (31).

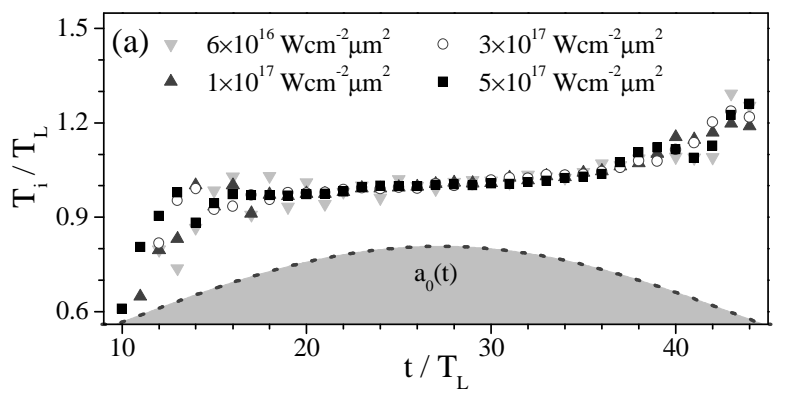

FIG. 22: Time dependence of the delay between the emission of two successive attosecond pulses, for four different peak laser intensities. In these CALDER simulations, $n_{e}^{\max }=110 n_{c}$ and $L=\lambda_{L} / 30$. The gray area shows the temporal profile of the laser pulse amplitude.

The origin of this discrepancy between this simple model and numerical observations can be traced back to one of the crude approximations made to evaluate $\tau_{e}$. In Sec. IV B, we approached the function $v_{r}\left(t_{r}\right)$ by a linear function. This approximation is justified when the most energetic Brunel electrons are not involved in CWE, but becomes too crude when the electrons that excite plasma oscillations in the density gradient have a velocity close to the maximum value (see Fig. 23(a)). This eventually becomes the case whatever the gradient scale length, when $a_{0}$ gets weak enough.

To solve this issue and properly describe the variation of $\tau_{e}$ with $a_{0}$, we have fitted $v_{r}\left(t_{r}\right)$ using the ad hoc analytical expression $v_{r}=v_{0} /\left(C+e^{\left(t_{0}-t_{r}\right) / \tau}\right)$. Figure 23 shows that, with a proper choice of the parameters $v_{0}, t_{0}$, $\tau$ and $C$, this provides a very good fit of $v_{r}\left(t_{r}\right)$, including around its maximum.

Using this fit and assuming, as in Sec. IVB, that $v_{r} \propto a_{0}$, a new expression for $\tau_{e}\left(a_{0}\right)$ can be derived. It is plotted in Fig. 23(b), for two different peak intensities, and the same laser envelope as in PIC simulations. We stress that these theoretical curves have been calculated without adjusting $t_{0}$ nor any other parameter. The agreement with PIC results is excellent. In particular, the fact that $\tau_{e}\left(a_{0}\right)$ is simply shifted without significant distortion when $a_{0}^{\max }$ is varied is well-reproduced by the analytical model. Note that in this improved model, the variation of $\tau_{e}$ with $a_{0}$ mostly originates from the variation of the return time of Brunel electrons to the overdense plasma.

This study shows that the main features of CWE can be reproduced analytically assuming that $v_{r}\left(t_{r}\right)$ is known. This is in particular sufficient to determine the emission time and hence the femtosecond chirp of CWE harmonics. In most cases a linear fit of this function is sufficient. However, a more accurate fit is needed when the laser intensity is so low that Brunel electrons involved in CWE have return velocities approaching the maximum of this function. 

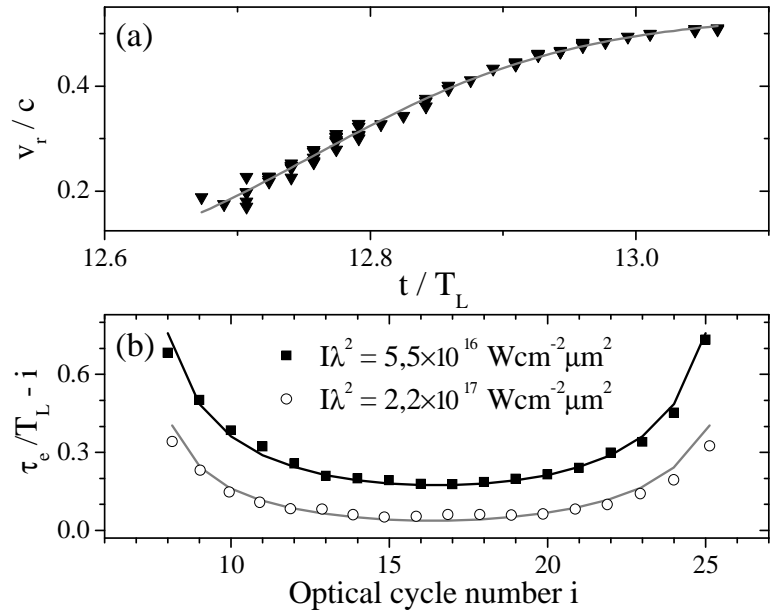

FIG. 23: Comparison of the emission times predicted by the analytical model, with the ones resulting from PIC simulations. (a) Fit of the function $v_{r}(t)$ obtained from a PIC simulation around $t \approx 13 T_{L}$ (same case as in Fig. 7). (b) Emission time $\tau_{e} / T_{L}-i$ as a function of the optical cycle number $i$ for two laser intensities. The points come from PIC simulations, while the lines are theoretical fits. The points corresponding to the lower intensity originates from the same simulation as (a).

\section{G. Conclusion}

In conclusion, Coherent Wake Emission is a mechanism of light generation by collective electron oscillations in a spatially-inhomogeneous plasmas, typically at a plasmavacuum interface. In the case of HHG on plasma mirrors, these oscillations are excited in the wake of laser-launched electron bunches that travel across the plasma, and emit light in the extreme ultraviolet spectral range. However, CWE can also be induced through other excitations schemes, and emit in very different frequency ranges, determined by the density of the emitting plasma. Plasma oscillations excited in underdense plasmas in the wake of femtosecond laser pulses can for instance emit terahertz pulses through CWE $[53,54]$. In a very different context, type III radio bursts originating from the interplanetary plasma $[65,66]$ might be due to CWE by plasma oscillations excited by energetic electrons produced during stellar flares.

We have shown here that the different steps of HHG on plasma mirrors by CWE, and the main features of the light emission, are now fairly well understood, at least semi-quantitatively. Two elements are now needed to go further in the analytical modeling of this process:

(i) A description of the shape and amplitude of the density peaks formed by the Brunel effect in the overdense part of the plasma.

(ii) A description of the transverse current distribution in the density gradient, taking into account refraction of the emitted light. As we have seen in sec. IV D 2, this is necessary for a satisfactory calculation of the light emission efficiency by plasma oscillations in the gradient.

While point (i) is specific to HHG on plasma mirror, solving point (ii) is crucial for a proper and quantitative description of CWE as a general light emission mechanism by inhomogeneous plasmas.

\section{RELATIVISTIC OSCILLATING MIRROR}

Harmonic generation on plasma mirrors at relativistic laser intensities has attracted considerable interest for the last fifteen years [26, 67-74]. In the past few years, this interest has been renewed as its unique potential for the generation of energetic attosecond [75] or even subattosecond [28] pulses of very short wavelengths was identified. In this relativistic interaction regime, harmonic generation on plasma mirrors is now commonly interpreted using the Relativistic Oscillating Mirror (ROM) model [25, 27-29, 76]. From a qualitative point of view, the idea underlying this model is simple. It attributes the generation of harmonic frequencies to a periodic Doppler effect induced on the reflected beam by the laser-driven relativistic oscillation of the plasma surface.

In the literature, different analytical approaches have been used to describe this process, using assumptions such as a step like interface that oscillates as a whole [27], or the cancellation of the tangential electric field [29] or vector potential [76] at the surface. Our analysis reveals that such assumptions are not crucial, but are simply different ways to account for the Doppler effect in analytical models.

\section{A. Observation in PIC simulations}

As an illustration of HHG in the relativistic regime, Fig. 24 shows the electron density and the attosecond pulse emission of a dense plasma driven by a $p$-polarized laser field, with a normalized vector potential $a_{0}=4(I=$ $3.4 \times 10^{19} \mathrm{~W} / \mathrm{cm}^{2}$ at $\left.800 \mathrm{~nm}\right)$. Dense jets of electrons are periodically pulled out of the plasma by the intense laser field. The density of these jets is however significantly lower than the bulk density. In other words, the plasma does not oscillate as a whole, as can be suggested by the oscillating mirror picture, but is rather strongly distorted by the laser field. The emission of attosecond pulses is observed precisely when these jets move outward with $v \approx c$. This figure therefore intuitively suggests that the associated high-frequencies result from the Doppler effect.

This is however far from obvious, and this simple observation should not be considered as a proof. In this regime, the transverse current distribution induced by the laser in the plasma is extremely complex. Strong temporal modulations of both $n_{e}$ and $\gamma$ occur. As we 


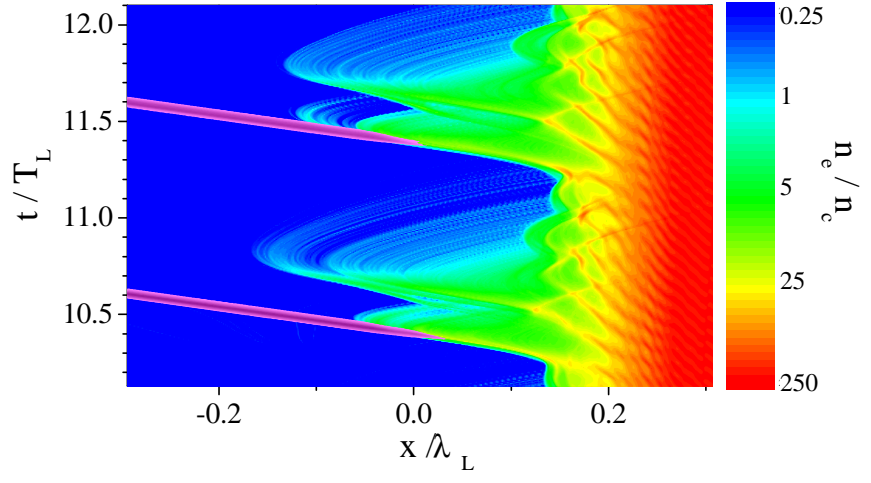

FIG. 24: (Color online) EUTERPE simulation of the Relativistic Oscillating Mirror process. The planar target is on the right side, while the laser arrives from left. The electron density $n_{e}$ (in $\log$ scale) corresponds to the blue to red scale, and the intensity of the electromagnetic field, frequency-filtered from harmonics 5 to 95 , to the purple one. The intensity is $I \lambda_{L}^{2}=2.2 \times 10^{19} \mathrm{~W} / \mathrm{cm}^{2} \mu \mathrm{m}^{2}$, the plasma density is $225 n_{c}$, and an exponential density gradient of scale length $\lambda_{L} / 20$ is initially imposed.

have seen in Sec. III E, this can directly lead to HHG, even in the absence of any Doppler effect. In addition, assuming that ROM is indeed the main contribution to HHG in this case leaves some features of Fig. 24 unexplained: while two outward excursions of the plasma surface occur in each optical cycle, both with relativistic velocities, only one attosecond pulse is emitted in this time interval. Thus, an outward excursion of the plasma 'surface' at relativistic velocity does not necessarily imply the efficient generation of high frequencies.

To clarify these issues, Sec. VB to VE provides a detailed analysis of the Doppler effect on moving mirrors. Sec. V G is then dedicated to a parametric study of ROM, and finally, Sec. VH focuses on the phase properties of the emission.

\section{B. Mirror in uniform motion}

We first analyze the Doppler effect occurring when a wave $\psi(x, t)=\psi_{0} \cos (\omega t-k x)$ reflects on a mirror moving with a constant velocity. As we will see, the formula for the Doppler shift is, in this case, the same for any kind of wave, including electromagnetic waves. It can therefore be established without any explicit reference to Special Relativity.

We restrict our discussion to the case of normal incidence for simplicity. The mirror moves with a velocity $v$ (with $v>0$ when the mirror moves toward the source) with respect to a reference frame where the wave has a phase velocity $v_{p}=\omega / k$, with the restriction $|v|<v_{p}$.

The field experienced by the mirror is given by $\psi(x=$ $-v t, t)=\psi_{0} \cos \left(\omega^{\prime} t\right)$, with $\omega^{\prime}=\omega\left(1+v / v_{p}\right)$. Currents are induced by this wave in the mirror, which thus behaves as a moving source of frequency $\omega^{\prime}$. During one oscillation period $T^{\prime}=2 \pi / \omega^{\prime}=\lambda^{\prime} / v_{p}$ of these currents, the mirror moves a distance $v T^{\prime}$. The emission of this source is therefore detected by a fixed observer at a wavelength $\lambda "=\lambda^{\prime}-v\left(\lambda^{\prime} / v_{p}\right)$, corresponding to a frequency $\omega "=$ $2 \pi v_{p} / \lambda^{\prime \prime}=\omega^{\prime} /\left(1-v / v_{p}\right)$.

The total Doppler shift of the reflected wave is thus $\omega " / \omega=\left(1+v / v_{p}\right) /\left(1-v / v_{p}\right)$. In the case of an electromagnetic wave, $v / v_{p}=v / c=\beta$ and

$$
\omega " / \omega=\alpha=\frac{1+\beta}{1-\beta}=(1+\beta)^{2} \gamma^{2}
$$

This is the well-known Doppler shift induced upon reflection on a moving mirror, with $\omega " / \omega \rightarrow 4 \gamma^{2}$ as $v \rightarrow c$ [77].

This simple demonstration gives a physical insight of the different terms involved in this frequency shift. The $1+\beta$ term is due to the fact that the mirror can be considered as an observer moving with respect to the source, which results in currents in the mirror at a frequency $\omega^{\prime}=\omega(1+\beta)$. This frequency is at most a factor of 2 higher than the initial frequency. Because the mirror, considered now as a source, is moving, its emission is observed at a frequency $\omega "=\omega^{\prime} /(1-\beta)$ different from the frequency $\omega^{\prime}$ of the currents. When $v \rightarrow+c$, this is the main contribution to the Doppler upshift. Note that a more general analysis, valid in the case of oblique incidence, can be found in Ref. [78].

\section{Doppler effect and retarded currents}

We have seen in Sec. III B that the field emitted by a $1 \mathrm{D}$ transverse current distribution is determined by the integral of this distribution along a light 'cone' (Eq. (4)). In this section, we show how this formula can account for the Doppler effect that occurs when the source is moving. To this end, we again consider a mirror in uniform motion, as discussed in Sec. VB.

We first consider a reference frame $R$, with coordinates $(x, t)$, where the mirror is fixed. It is exposed to a linearly-polarized, normally-incident electromagnetic plane wave of frequency $\omega_{L}$ propagating along $x$ from $-\infty$ toward $+\infty$. This wave induces a transverse current in the mirror along the polarization direction. Assuming that the mirror surface occupies the half-space $x \geq 0$, this current is given in complex representation by

$$
j_{t}(x, t)=\left\{\begin{array}{ll}
e^{i \omega_{L} t} e^{-x / \delta} & \text { for } x \geq 0 \\
0 & \text { for } x<0
\end{array},\right.
$$

where $\delta \approx c / \omega_{p}$ is the skin depth, with $\omega_{p}$ the plasma frequency in frame $R$.

We now consider the same system in a frame $R^{\prime}$, with coordinates $\left(x^{\prime}, t^{\prime}\right)$, moving with respect to $R$ at a velocity $v=\beta c$, with $\beta>0$ when the mirror moves toward the light source in frame $R^{\prime}$. According to Special Relativity, the frequency of the light wave incident on the mirror in this frame is $\omega=\gamma(1-\beta) \omega_{L}$. As 
the transverse current distribution lies in the $\left(\mathbf{e}_{y}, \mathbf{e}_{z}\right)$ plane, its expression $j_{t}^{\prime}\left(x^{\prime}, t^{\prime}\right)$ in frame $R^{\prime}$ is simply obtained by expressing the variables $(x, t)$ as a function of $\left(x^{\prime}, t^{\prime}\right)$ in Eq. (34), using the Lorentz transformation $(x, c t)=\left(\gamma\left(x^{\prime}+\beta c t^{\prime}\right), \gamma\left(c t^{\prime}+\beta x^{\prime}\right)\right)$. This leads to

$$
j_{t}^{\prime}\left(x^{\prime}, t^{\prime}\right)=\left\{\begin{array}{ll}
e^{i \frac{\omega t^{\prime}+\beta k x^{\prime}}{1-\beta}} e^{-\frac{x^{\prime}+v t^{\prime}}{\delta / \gamma}} & \text { for } x^{\prime} \geq-v t^{\prime} \\
0 & \text { for } x^{\prime}<-v t^{\prime}
\end{array},\right.
$$

where $k=\omega / c$.

The field radiated by this distribution at $x=-\infty$, which corresponds to the light reflected by the plasma, is then given by

$$
E\left(t^{\prime}\right)=\mu_{0} \int_{-\infty}^{+\infty} d x^{\prime} j_{t}^{\prime}\left(x^{\prime}, t^{\prime}-x^{\prime} / c\right)
$$

Substituting the new variable $x^{\prime \prime}=(1-\beta) x^{\prime}+v t^{\prime}$ in this integral, it can be shown that the frequency of the reflected wave $E(t)$ in frame $R^{\prime}$ is $\omega^{\prime \prime}=\omega(1+\beta) /(1-\beta)$, in agreement with the Doppler shift given by Eq. (33).

We can now compare the frequency $\omega^{\prime \prime}$ of this reflected wave to the frequency $\omega^{\prime}$ of the current source in $R^{\prime}$, i.e. the frequency of the current that would be seen by an observer that would follow the mirror. Setting $x^{\prime}=-v t^{\prime}$ (the position of the mirror surface) in Eq. (35), we obtain $\omega^{\prime}=(1+\beta) \omega$. This result thus supports the simple analysis of the previous section: because of the mirror motion, the current in the skin layer has a frequency which is shifted by a factor $1+\beta$ with respect to the incident wave frequency $\omega$. This moving current distribution then radiates at a frequency which is further shifted by a factor $(1-\beta)^{-1}$.

In the formalism of retarded currents, this last shift is due to the integration along light cones of slope $c$. The qualitative origin of this effect appears very clearly in Fig. 25, which shows the current distribution for (a) $v=0$ and (b) $v=0.5 c$. In (a), where the source is fixed, the frequency of the emitted field is identical to that of the current source, despite the integration along a tilted line. In (b), where the source now moves with $\beta=0.5$, the current distribution is now also tilted in the $(x, t)$ space, and as a result two integration lines spaced by $d t=T^{\prime}=2 \pi / \omega^{\prime}$ span two oscillations of the current $j_{t}^{\prime}$, while they only spanned one when $v=0$. Therefore, an observer now measures two oscillations of the field in a time $d t=T^{\prime}$, instead of one when $v=0$. The frequency of the signal has been increased by a factor of two. This is the origin of the $(1-\beta)^{-1}$ term in the Doppler effect.

\section{Doppler effect in reciprocal space}

We now analyze how the Doppler effect appears in the Fourier space $(k, \omega)$, focusing on Fig. 25 (c-d) which show the Fourier transforms $\hat{\hat{j}}_{t}(k, \omega)$ of the two transverse current distributions plotted in Fig. 25(a-b).
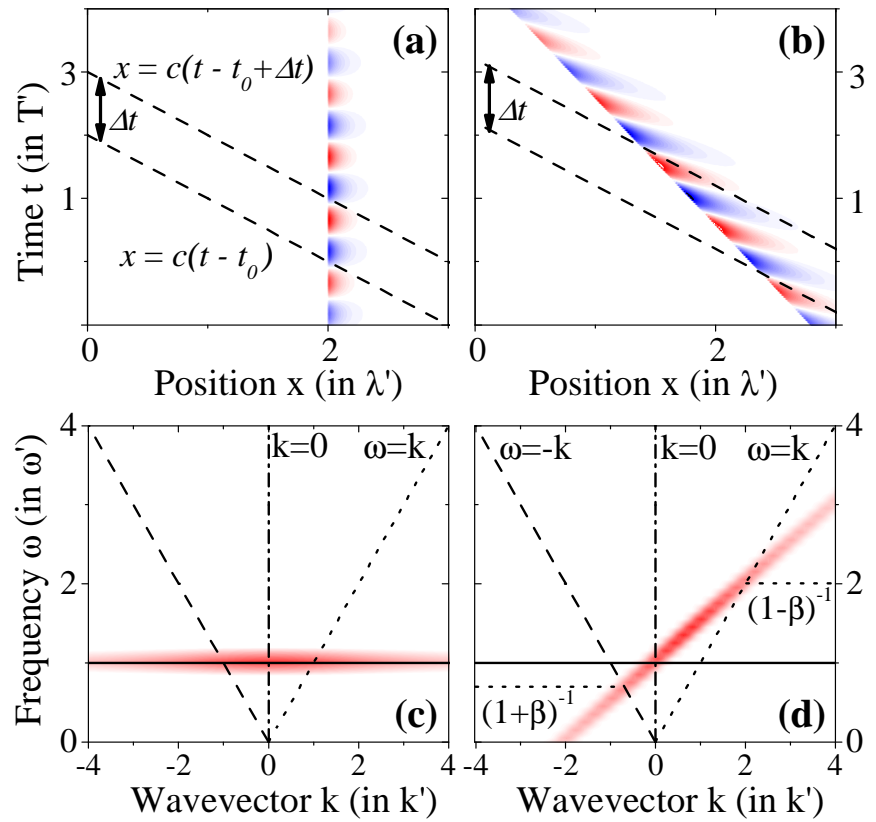

FIG. 25: (Color online) Two cases of transverse current distributions $j_{t}(x, t)$ in a mirror exposed to an electromagnetic wave in normal incidence. In (a), the mirror is immobile $(\beta=0)$, while in $(\mathrm{b})$, the mirror moves with $\beta=0.5$. In both cases, the frequency of the current induced by the wave in the mirror is $\omega^{\prime}=(1+\beta) \omega$, where $\omega$ is the frequency of the incident wave. Panel (c) and (d) show the respective 2D Fourier-transforms $\hat{\hat{j}}_{t}(k, \omega)$ of these distributions. The spectrum reflected by the mirror is given by a lineout of these distributions along the line $\omega=c k$ (with $c=1$ in the units of these graphs), while the instantaneous spectrum corresponds to a lineout along $k=0$. The emission of the mirror in the forward direction (along the direction of the incident light) is given by a lineout along $\omega=-c k$.

In the first case, $\hat{\hat{j}}_{t}$ is a narrow horizontal line parallel to the $\omega$ axis, because the current distribution is an oscillating function of time, and is well-localized in space. The Fourier transform of the current distribution when $v \neq 0$ is similar, but the line is now rotated in the $(k, \omega)$ space. This is because a current distribution of the form $j_{t}(x, t)=j_{t}^{0}(x, t-x / v)$ has a Fourier transform with respect to time given by $\hat{j}_{t}(x, \omega)=\hat{j}_{t}^{0}(x, \omega) e^{i \omega / v x}$. This leads to $\hat{\hat{j}}_{t}(k, \omega)=\hat{\hat{j}}_{t}^{0}(k-\omega / v, \omega)$, i.e. to spectral structures localized along a line of slope $v$.

According to Sec. III C, the spectrum of the reflected wave is given by the intersection of this distribution with the line $\omega=c k$ of slope $c$. Fig. 25(c-d) clearly show that this intersection moves in frequency when $v \neq 0$. This shift corresponds to the Doppler shift between the frequency of the reflected wave and that of the current distribution. As expected, when $v \rightarrow c$, this intersection moves to infinity, and so does the frequency $\omega^{\prime \prime}$.

Let us now consider the instantaneous spectrum that would be radiated by the source in the absence of retardation effect. According to Sec. III C, this spectrum is 
given by a lineout of $\hat{\hat{j}}_{t}(k, \omega)$ along the line defined by $k=0$. In the case of Fig. 25(c-d) the instantaneous spectrum is thus peaked at $\omega=\omega^{\prime}=(1+\beta) \omega_{L}$, i.e. at the frequency of the current induced in the mirror by the incident wave, whatever the motion of the mirror. Thus, for a known current distribution moving toward the observer, this procedure makes it possible to eliminate the Doppler effect induced on the emission by the source motion, i.e. the $(1-\beta)^{-1}$ term in Eq. (33). In the case of a mirror, the $1+\beta$ term of course remains, but this can only account for a factor of at most two in the frequency shift, much smaller than the $(1-\beta)^{-1}$ term when $\beta \rightarrow 1$.

In conclusion, in the case of a moving mirror, the dominant contribution to the Doppler effect is characterized by a difference between the instantaneous spectrum and the real spectrum. Since these spectra are respectively given by lineouts of $\hat{j}_{t}(k, \omega)$ along $k=0$ and $\omega=c k$, the shape of this function in the $(k, \omega)$ reciprocal space provides a simple way to detect at a glance the occurrence of such a Doppler effect.

\section{E. Doppler effect in HHG}

We now apply the analysis of Secs. V C and VD to assess the importance of the Doppler effect in the case of a plasma mirror exposed to a relativistically intense laser field. The transverse current distribution is then much more complex, and, in the present state of our knowledge, cannot be calculated analytically. It can however be directly obtained from PIC simulations. A transverse current distribution $j_{t}(x, t)$, resulting from an EUTERPE simulation in the relativistic regime, is shown in Fig. 26, and its 2D Fourier transform $\hat{\hat{j}}_{t}(k, \omega)$ in Fig. 27. The waveform of the reflected electromagnetic field is shown in the left panel of Fig. 26, and the corresponding spectrum, comprising high harmonic orders of several tens, in Fig. 28. Note that a low density plasma is considered $\left(n_{e}=6 n_{c}\right)$ to avoid any contribution of CWE to the harmonic signal.

A first qualitative analysis can be performed in the $(x, t)$ space. The current distribution $j_{t}(x, t)$ presents some rather sharp structures, moving at a velocity close to $c$ toward vacuum. They are induced by the outgoing electrons bunches mentioned earlier, and already observed in Fig. 24. The emitted field at point $x_{0}$ at different times $t_{0}$, is obtained integrating this distribution along the 1D light cones $\left(x-x_{0}\right)=c\left(t-t_{0}\right)$. The left panel in Fig. 26 shows that it exhibits fast temporal variations, which occur when the integration line crosses the parts of the current distribution which have slope close to $c$. This corresponds to the Doppler effect induced by the moving mirror.

As emphasized in Sec. VD, the importance of this Doppler effect can be more readily assessed by turning to the reciprocal space $(k, \omega)$. In Fig. 27, the emitted spectrum $S(\omega)$, given by the lineout of $\hat{j}_{t}(k, \omega)$ along the

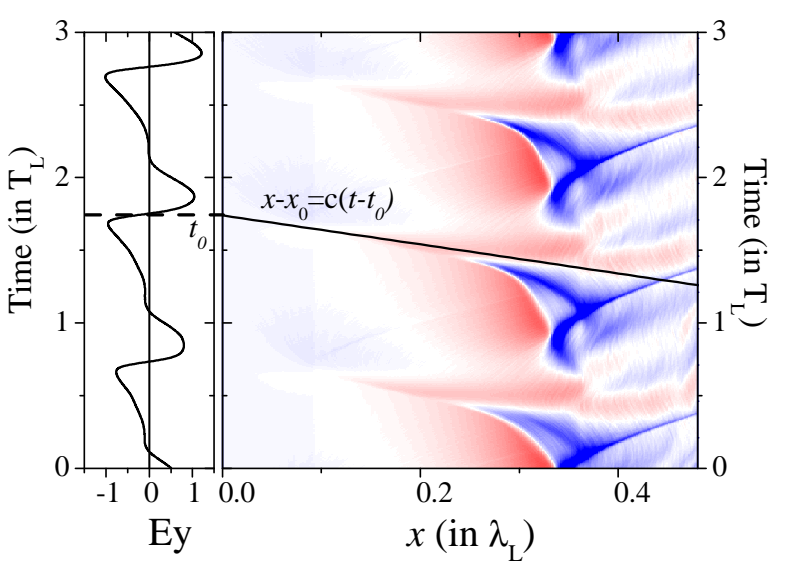

FIG. 26: (Color online) Transverse current distribution along the $y$-axis, in the boosted frame, induced by a $p$-polarized laser field of $I \lambda_{L}^{2}=1.3 \cdot 10^{18} \mathrm{~W} / \mathrm{cm}^{2} \mu \mathrm{m}^{2}$, impinging a plasma with $n_{e}^{\max }=6 n_{c}$ and $L=\lambda_{L} / 20$. The integration line to calculate the electric field at a given time $t_{0}$ is shown. The left panel shows the waveform of the electric field reflected by this plasma mirror.

line $\omega=c k$, comprises many more harmonic orders than the instantaneous spectrum $\tilde{S}(\omega)$, given by the $k=0$ lineout. This is a clear indication of the crucial role of the Doppler effect.

To illustrate this point more quantitatively, in Fig. 28, these two lineouts are compared to the reflected light spectrum $S_{P I C}(\omega)$ directly provided by the PIC code, from the step-by-step resolution of Maxwell's equations on the spatio-temporal mesh of the simulation. The agreement between $S_{P I C}(\omega)$ and $S(\omega)$ is excellent, thus validating our calculation of the field using the transverse current provided by the PIC code. The harmonic signal in $\tilde{S}(\omega)$ drops by orders of magnitude compared to $S(\omega)$ for harmonic orders beyond $n \approx 3-5$. This comparison shows without any ambiguity that the harmonic content of the reflected light is in this case almost entirely due to the Doppler effect induced by the source motion.

To support this analysis, it is also instructive to compare the current distribution $\hat{j}_{t}(k, \omega)$ corresponding to ROM (Fig. 27), to the one corresponding to CWE (Fig. 16). In the later case, there is only minor differences between the harmonic intensities obtained in the emitted and instantaneous spectra. This is because the emitted harmonic spectrum results from a collection of locally harmonic currents, with a broad range of frequencies distributed at different locations in the plasma. The Doppler effect then plays absolutely no role.

To get a better understanding of what factors determine the shape of $\hat{\hat{j}}_{t}(k, \omega)$ in the case of Fig. 27, we consider the following empirical oscillating current distribu- 


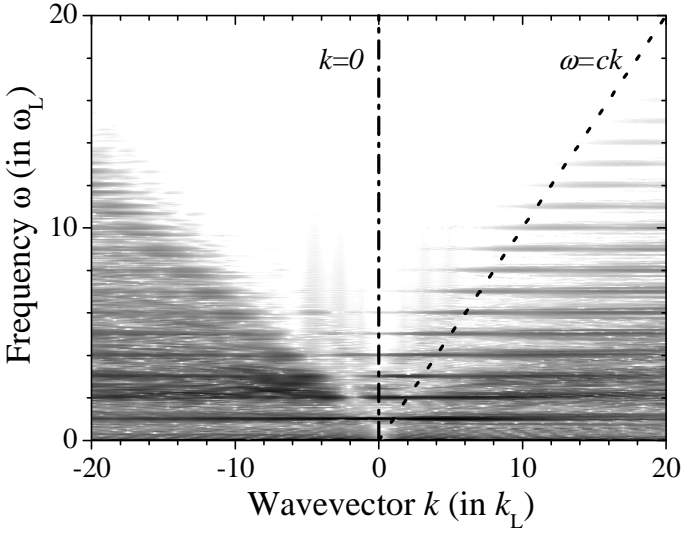

FIG. 27: 2D Fourier transform $\hat{\hat{j}}_{t}(k, \omega)$ of the distribution $j_{t}(x, t)$ shown in Fig. 26. The reflected spectrum is given by a line-out along $\omega=c k$, and the instantaneous spectrum along $k=0$.

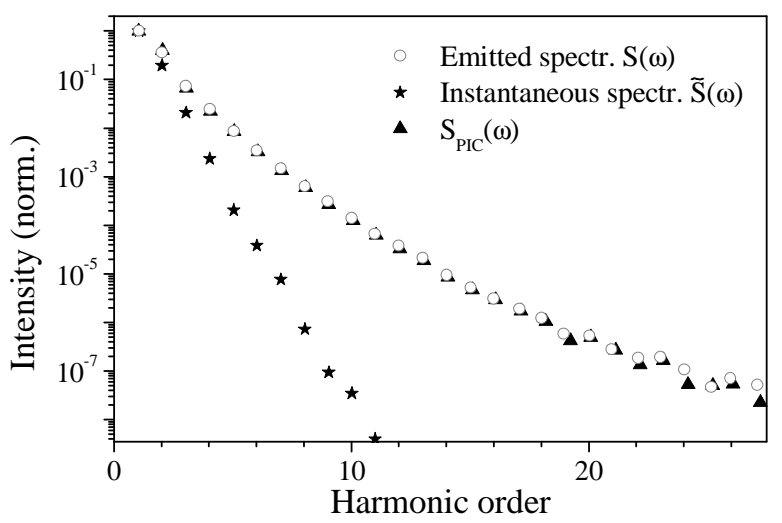

FIG. 28: Emitted spectrum $S(\omega)$ (hollow circles) and instantaneous spectrum $\tilde{S}(\omega)$ (stars) calculated from the transverse current distribution of Fig. 26 using Eqs. (5) and (7) of Sec. III. To improve visibility, only the peak intensity of individual harmonics is plotted. The emitted spectrum matches almost perfectly the one directly provided by EUTERPE (black triangles). When retardation effects are suppressed, the harmonic signal is strongly reduced for orders $>3$.

tions $j_{t}(x, t)$, which roughly mimics the one of Fig. 26:

$j_{t}=\left\{\begin{array}{ll}e^{\frac{-\left(x-x_{s}(t)\right)}{\delta}} \sum_{n=1}^{n_{\max }} a_{n} \cos \left(n \omega_{L} t+\varphi_{n}\right) & \text { for } x \geq x_{s}(t) \\ 0 & \text { for } x<x_{s}(t)\end{array}\right.$,

with $x_{s}(t)=\left(v_{M} / \omega_{L}\right) \cos \left(\omega_{L} t\right)$. Such distributions are plotted in Fig. 29, for (a) $v_{M}=0$, (b) $v_{M}=0.5 \mathrm{c}$ and (c) $v_{M}=0.95 \mathrm{c}$. The corresponding Fourier-transforms $\hat{\hat{j}}_{t}(k, \omega)$ are displayed in Fig. 29(d-e-f). In these examples, $n_{\max }=3$, with $a_{1}=6, a_{2}=3, a_{3}=1$ and $\varphi_{1}=\pi / 2, \varphi_{2}=0, \varphi_{3}=0$. When $v_{M}=0$ (Fig. 29(ad)), $j_{t}(x, t)$ simply corresponds to a local anharmonic current, with three frequency modes. The corresponding peaks in $\hat{\hat{j}}_{t}(k, \omega)$ lie along the $k=0$ axis, like in the
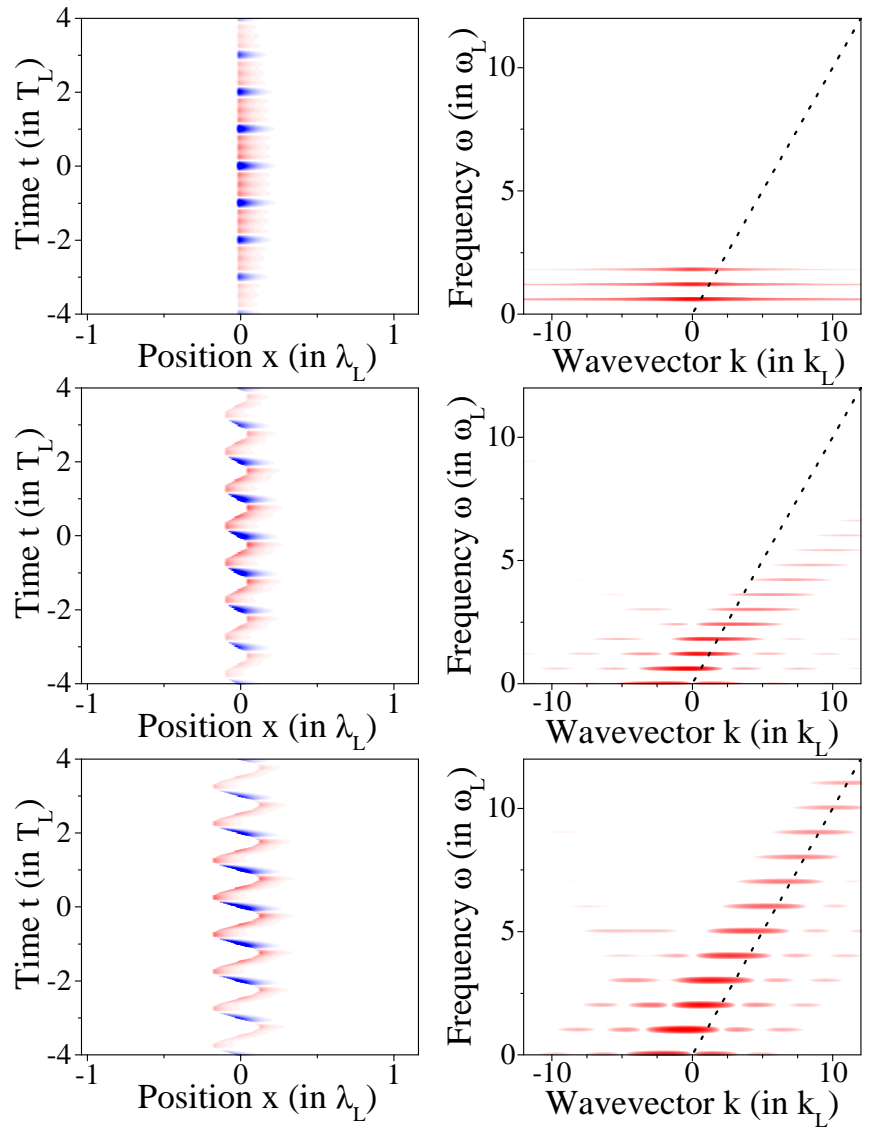

FIG. 29: (Color online) Three cases of model transverse current distributions $j_{t}(x, t)$ (a-b-c), given by Eq. (37), with their respective $2 \mathrm{D}$ Fourier transforms (d-e-f). In (a), the current distribution does not move along the $x$ axis, while in (b) and (c) it oscillates with peak velocities of respectively $0.5 c$ and $0.95 c$.

simple case of the immobile mirror of Fig. 25(c). When $v_{M} \neq 0$ (Fig. 29(b-c)), a Doppler effect appears very clearly in $\hat{\hat{j}}_{t}(k, \omega)$ (Fig. 29(e-f)). While the instantaneous spectrum, along the $k=0$ axis, still consists in three frequency modes, the emitted spectrum, along $\omega=c k$, comprises many more harmonic orders. Comparing cases (e) and (f) shows that, in analogy with the case of a mirror in uniform motion (Fig. 25(d)), the slope of the tilted structure observed in this distribution is determined by the peak velocity $v_{M}$ encountered in the current distribution $j_{t}(x, t)$.

The same thing occurs in the case of laser-driven oscillating mirror. The slope of the tilted structure observed in the distribution of Fig. 27 is determined by the peak velocity encountered in the current distribution $j_{t}(x, t)$, which thus has a crucial influence on the extension of the harmonic spectrum. The harmonic frequencies that are observed along the $k=0$ axis, and that lead to the harmonic signal remaining in the instantaneous spectrum $\tilde{S}(\omega)$, are due to a local anharmonicity of the transverse current. Several factors can contribute to this anharmon- 
icty, such as the saturation of the electron velocity in the relativistic regime (see Sec. III E).

In realistic cases, the harmonic spectrum is however not fully determined by the peak velocity. Two features of the electron jets responsible for the relativistic structures in $j_{t}(x, t)$ can, in particular, influence the emitted field:

(i) the phase relationship between currents associated to different parts of these jets,

(ii) the duration and the spatial extension of the jets.

Figure 24 illustrates the first point: despite its relativistic velocity, the second electron bunch of each optical cycle does not generate efficiently high order harmonics, because the fields radiated by the different parts of this jet interfere destructively. The second point is, in principle, more beneficial for efficient HHG. The transient character of the Doppler effect, due to the sub-laser cycle duration of the jets, tends to increase the harmonic spectral cut-off compared to the $4 \gamma^{2}$ law, valid for a mirror in uniform motion only. The more localized in time the relativistic bunch is, the broader $\hat{\hat{j}}_{t}(k, \omega)$ is along the $\omega$ axis in $(k, \omega)$ space, and the more harmonics are generated. The spatial localization of the bunches induces a similar effect along the $k$ axis. As first emphasized in [29], due to this type of effect, the emitted frequency bandwidth tends to become proportional to $\gamma^{3}$ in the limit of an ultra-relativistic charge in non-uniform motion, as is well known for synchrotron radiation of moving charges. However, given the complexity of the current distributions considered here, these is no reason to expect that such a simple law exactly holds for plasma mirrors - and indeed, as we will see in Sec. V G, it is not fully supported by a parametric study of this process.

\section{F. The $\gamma$-spikes model}

Within our previous analysis, a detailed knowledge of the transverse current distribution is needed to calculate the harmonic emission of the plasma. Determining this distribution analytically in the relativistic interaction regime is extremely challenging, and we therefore had to turn to PIC simulations to be able to study HHG from the ROM process. The $\gamma$-spikes model, also called the Baeva-Gordienko-Pukhov (BGP) theory and proposed in Ref. [29], uses a totally different approach based on a boundary condition. We summarize this general approach and its main conclusions, and then discuss its limitations.

\section{Summary and main results}

Instead of trying to determine the current distribution, the starting point of the BGP theory is the assumption that a so-called apparent reflection point (ARP) can be found, with position $x_{A R P}(t)$, such that for any time $t$

$$
E_{y}^{i}\left(x_{A R P}(t), t\right)+E_{y}^{r}\left(x_{A R P}(t), t\right)=0
$$

where $E_{y}^{i}$ and $E_{y}^{r}$ are respectively the component of the incident and reflected fields along the target surface. The authors emphasize that this ARP is not necessarily a real physical point where the total tangential electric field actually cancels. Qualitatively speaking, this generalizes the usual boundary condition at the surface of a perfect fixed mirror, to the case of a moving mirror, which effect is then to induce a simple phase modulation on the reflected beam. This boundary condition is as a matter of fact an assumption, since no complete demonstration has been provided to support it [29]. The analysis has initially been proposed for normal incidence of the laser, but one might expect it to equally apply to any incidence angle by a simple transformation to the boosted frame.

Using this boundary condition, the authors analytically derive two main, and supposedly universal properties of the emitted harmonic spectrum, in the limit of ultra-relativistic laser intensities. First, the harmonic spectral intensity is predicted to decay as $n_{H}^{-8 / 3}$ with harmonic order $n_{H}$. Second, this power law decay extends up to a maximum frequency $\omega_{c}$ that scales as $\gamma^{3}$, where $\gamma$ is the maximum Lorentz factor of the apparent reflection point.

Thus, an important achievement of this theory was to point out, for the first time, that the frequency cutoff of the harmonic spectrum does not follow a $\gamma^{2}$ scaling as in the case of a mirror in uniform relativistic motion (see Eq. (33)), but potentially reaches much higher frequencies due to the $\gamma^{3}$ scaling. Interestingly, this corresponds to the general scaling expected for a charge in arbitrary ultrarelativistic motion [41]: in the BGP model, electrons at the plasma surface thus behave as a bunch of ultrarelativistic electrons radiating coherently. This enhanced spectral width is due to the fact that the Doppler-induced frequency upshift of the reflected light only occurs during the small fraction of the laser optical cycle, of order $1 / \gamma$, in which the Lorentz factor $\gamma$ is maximum.

Quite remarkably, a good agreement between these theoretical predictions and experiments in oblique incidence and $p$-polarization has been reported in Refs. [73] and [74], which describe experimental results obtained with the VULCAN laser up to $a_{0} \approx 10$.

\section{Limitations of the theory}

However, an essential result of our detailed study of the ROM process is that the boundary condition (38) is generally not fulfilled in the relativistic regime, in particular in experimentally relevant interaction conditions (oblique incidence and finite density gradient scale length). The reason for this is illustrated in Fig. 30(a), which compares $E_{y}^{i}(t)$ to $E_{y}^{r}(t)$ in typical interaction conditions leading to 
HHG by ROM. We observe that the oscillation amplitude of $E_{y}^{r}(t)$ is almost a factor of 2 larger than that of $E_{y}^{i}(t)$. This is because the Doppler effect results in a temporal redistribution of the electromagnetic energy within each laser optical cycle, leading to a higher energy density at certain times -just when the frequency upshift occurswhile the total energy per optical cycle remains the same (in the absence of absorption). This effect is clearly observed in Fig. 30(b), which compares the temporal evolution of the instantaneous energy density in the incident and reflected fields, at the sub-laser-cycle time scale. A similar effect is actually observed in most simulations of ROM.

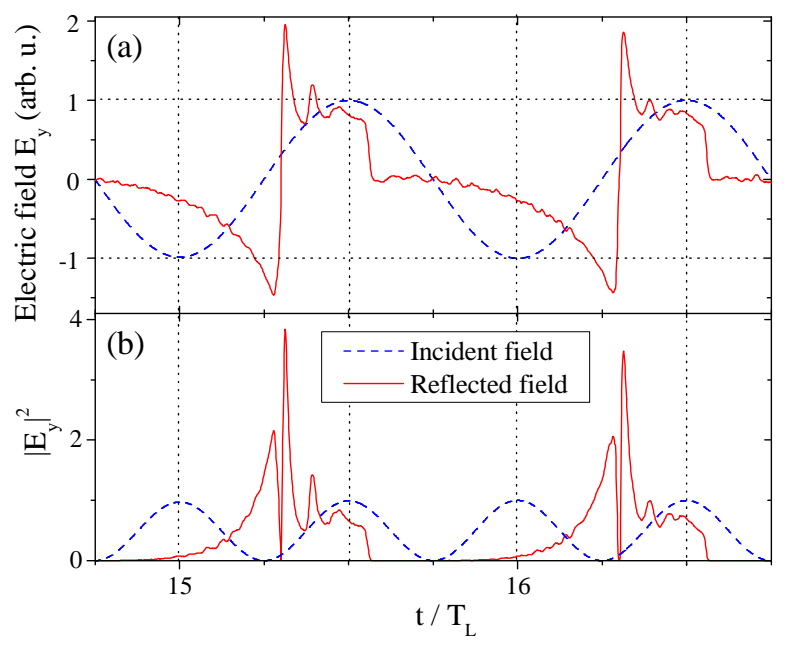

FIG. 30: Time redistribution of the laser field energy. (a) Comparison of the laser electric field before and after reflection on a plasma mirror, for $a_{0}=65, n_{e}^{\max }=225 n_{c}$, $L=\lambda_{L} / 20$, and incidence angle $45^{\circ}$. (b) Square of the incident/reflected laser electric fields $\left|E_{y}\right|^{2}$, as a function of time. This function is proportional to the instantaneous energy density in the field.

Whatever the motion of the hypothetical apparent reflection point, the equality (38) at the root of the BGP theory is impossible to fulfill for all times when $\left|E_{y}^{r}(t)\right|>\max \left|E_{y}^{i}\right|$. In other words, it is impossible to go from $E_{y}^{i}(t)$ to $E_{y}^{r}(t)$ by a mere phase modulation in such a case. Since these are precisely the times where harmonic generation occurs, this equation, and hence the theory derived from it, are inadequate to describe HHG in this regime. This severe limitation of the theory has recently been put forward by A. Pukhov and coauthors in Refs. [79, 80]. The parametric study of ROM that follows confirms that the BGP model fails to reproduce the results of most PIC simulations of ROM in oblique incidence (p-polarization), as already emphasized in Refs. [81, 82].

In summary, the BGP theory provides an original approach that lead to the first fully analytical decription of the ROM process in the ultrarelativistic regime, and the only experiments performed so far in the ultrarelatistic regime tend to support its main predictions $[73,74]$.
However, detailed inspection of numerical simulations show that the boundary condition used as a starting point for this theory is in many cases violated. This appears as a serious shortcoming of the theory, which, despite the insight it has provided, clearly does not cover all aspects of the interaction. Further numerical and theoretical studies will thus be necessary to determine the range of applicability of the BGP theory.

\section{G. Parametric study}

As in the case of Coherent Wake Emission, we now investigate how the laser peak intensity, the gradient scale length or the incidence angle affect the emission. The results presented in this section have been obtained with the code EUTERPE, for an exponential gradient with $n_{e}^{\max }=225 n_{c}$.

\section{Laser intensity}

We analyze first the influence of the laser peak intensity $I$. Figure 31(a) displays four harmonic spectra obtained for $3 \times 10^{18} \mathrm{~W} / \mathrm{cm}^{2} \mu \mathrm{m}^{2} \leq I \lambda_{L}^{2} \leq 3 \times 10^{21}$ $\mathrm{W} / \mathrm{cm}^{2} \mu \mathrm{m}^{2}$. We see clearly that the generation efficiency and the number of emitted harmonics increase with $I$. Further we observe in Fig. 31(b), which shows the generation efficiency of three different harmonics as a function of $I$, that the harmonic signal increases rapidly with laser intensity for $I \lambda_{L}^{2} \lesssim 10^{20} \mathrm{~W} / \mathrm{cm}^{2} \mu \mathrm{m}^{2}$, and then tends to saturate for very high intensities. Harmonic spectra thus converge towards a high-intensity limit.

According to the BGP theory discussed in Sec. VF, this limit should be proportional to $n_{H}^{-8 / 3}$. Figure 31 (a) shows however that this function gives only an order-ofmagnitude of the limit. This shortcoming of the theory is confirmed by Fig. 32, which illustrates that the power law $n_{H}^{-8 / 3}$ does not apply at all in the more realistic case where ion motion is taken into account.

The second prediction of the BGP theory, that is a frequency cutoff that varies as $\gamma^{3}$, is harder to check numerically (and even more experimentally), because $\gamma$ is the maximum Lorentz factor of the ARP point which has no physical reality (see Sec V F). Defining the cutoff $\omega_{c}$ as the frequency at which the spectrum starts to decrease significantly faster than $n_{H}^{-8 / 3}$, we roughly measure in Fig. 31(a), $\omega_{c} \propto I^{0.6 \pm 0.1}$. Then, assuming that $\omega_{c} \propto \gamma^{3}$, we find that $\gamma \propto I^{0.2}$. This is very different from the scaling $\gamma \propto I^{0.5}$ which is sometimes inferred assuming that the apparent reflection point behaves as would do a free electron in the incident laser field [74]. This discrepancy can either be due to the fact that the BGP cut-off does not match numerical results, or to the fact that $\gamma$ indeed does not vary as $I^{0.5}$. Whatever the reason, the conclusion of this analysis is that there is is no way to check unambiguously in experiments the cut-off predicted by 

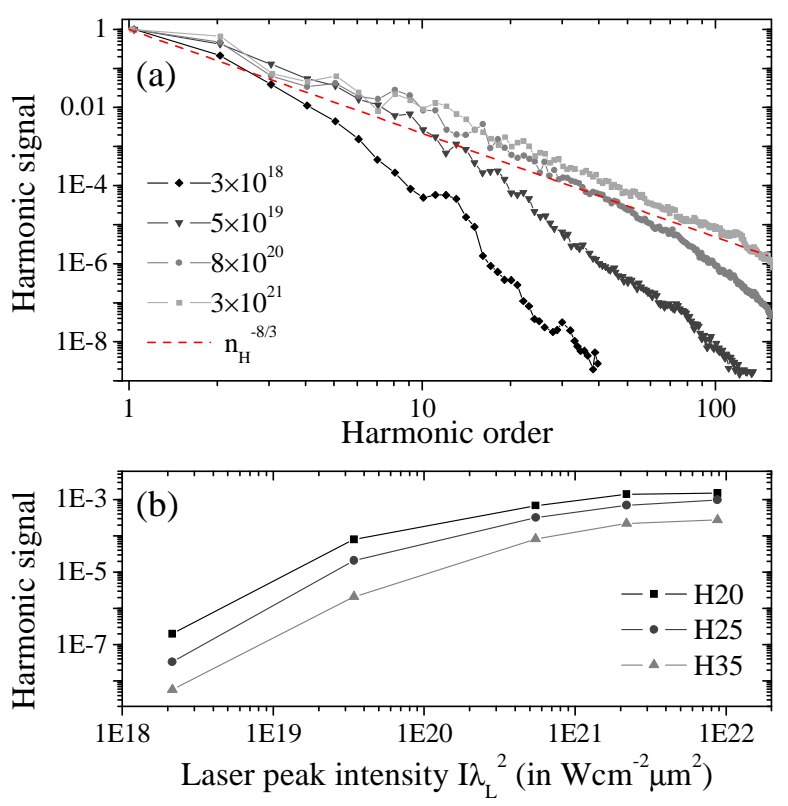

FIG. 31: (Color online) Influence of the laser peak intensity on the generation efficiency of ROM harmonics. (a) Spectra for 4 different laser intensities (in $\mathrm{W} / \mathrm{cm}^{2} \mu \mathrm{m}^{2}$ ), $L=\lambda_{L} / 20$ and $\theta=45^{\circ}$. The points correspond to the peak intensities of individual harmonics. The dashed line is the function $n_{H}^{-2.66}$. Solid lines are visual guides. (b) Variation with the laser intensity of the generation efficiency for harmonics 20, 25 and 35 .

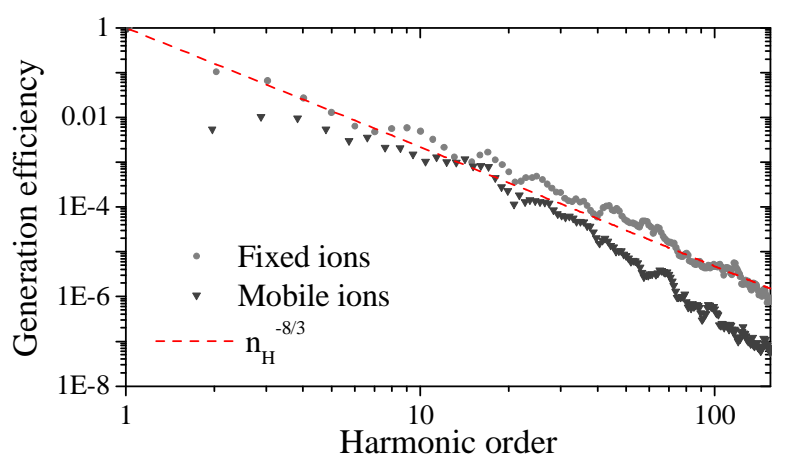

FIG. 32: Comparison of harmonic spectra for fixed and mobiles ions. In this case, $I \lambda_{L}^{2}=2 \times 10^{21} \mathrm{~W} / \mathrm{cm}^{2} \mu \mathrm{m}^{2}, \theta=45^{\circ}$, and initially $L=\lambda_{L} / 25$.

the BGP theory, since $\gamma$ cannot be measured, and since this theory does not give any prediction for the function $\gamma(I)$.

\section{Density gradient scale length}

We now focus on the influence of the density gradient scale length $L$. Figure 33 shows that an increase of $L$, has a similar effect to a rise of $I$, that is, an increase of the generation efficiency and of the harmonic spectrum extension. This is because for long density gradients the laser field, which is reflected around the critical surface, interacts with an almost critical plasma, whereas for very short $L$, it interacts with a solid density plasma. Because the ion recalling force is proportional to $\omega_{p}^{2} \propto n_{e}$, electrons can actually gain more energy in the laser field for longer gradient scale lengths. The generation efficiency can be thus enhanced equivalently by an increase of $I$ or $L$. It is worth mentioning that because the whole emission process happens in an area close to the critical surface, the form of the gradient is not expected to really matter in ROM. Both the form and the length of the gradient can, however, have some influence on the spectral and spatial properties of the laser before it reaches the critical surface, and can hence indirectly affect the emission.
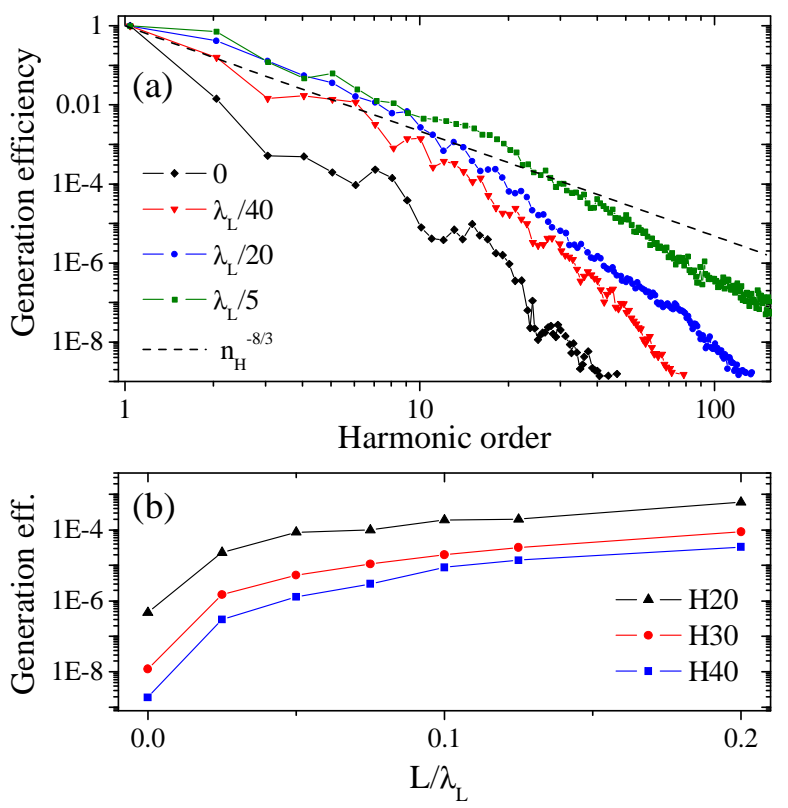

FIG. 33: (Color online) Influence of the gradient scale length on the generation efficiency of ROM harmonics. (a) Spectra for 4 different gradient scale lengths, $I \lambda_{L}^{2}=2 \times 10^{19}$ $\mathrm{W} / \mathrm{cm}^{2} \mu \mathrm{m}^{2}$ and $\theta=45^{\circ}$. (b) Variation with $L$ of the generation efficiency of harmonics 20,30 and 40.

\section{Incidence angle}

We pursue the parametric study by investigating the role of the incidence angle $\theta$. In Fig. 34, $\theta$ varies in the range $0^{\circ}-75^{\circ}$, while the laser energy is constant $(I \propto$ $\cos \theta)$. The overall generation efficiency first increases with the incidence angle, reaches a maximum for $\theta \approx 55^{\circ}$, and then decreases steeply with $\theta$.

The existence of an optimal angle and of a sharper decrease of the generation efficiency for angles above the optimum are strong evidence of the predominant role played by the component of the electric field along the plasma normal. Actually, for moderately relativistic laser intensities, electrons involved in the ROM process are, 
to a large extent, accelerated by the Brunel mechanism. Their velocities are thus proportional to $E_{x} \propto \sin \theta \sqrt{\cos \theta}$ which is asymmetric in $\theta$ and maximal for $\theta=55^{\circ}$. This simple calculation does not however account for the actual complexity of the plasma dynamics and the resulting angular dependence of the harmonic spectrum. For instance, it fails to explain why, in Fig. 34, the spectra obtained for $\theta=60^{\circ}$ and $\theta=45^{\circ}$ cross each other around $n_{H}=60$. The influence of the magnetic field is expected to rise with the laser intensity, and this could influence the results of Fig. 34 .

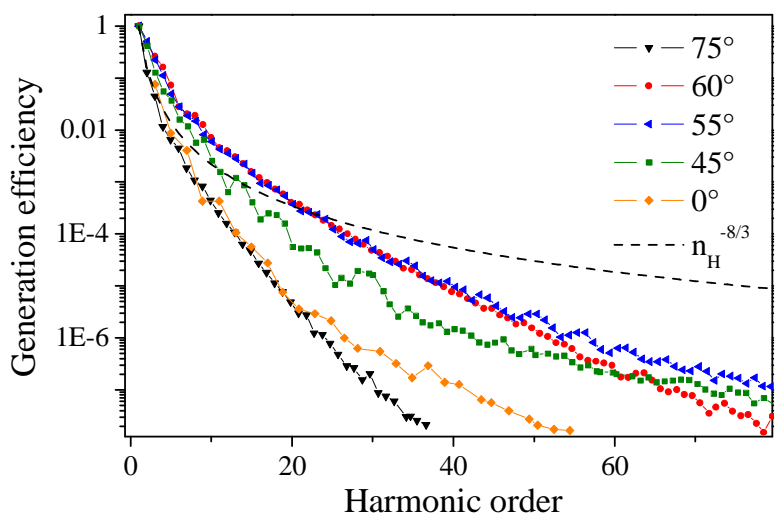

FIG. 34: (Color online) Influence of the incidence angle on the generation efficiency of ROM. In these simulations, $I \lambda_{L}^{2}=$ $2\left(\cos \theta / \cos 45^{\circ}\right) \times 10^{19} \mathrm{~W} / \mathrm{cm}^{2} \mu \mathrm{m}^{2}$ and $L=\lambda_{L} / 20$. An angle of $0^{\circ}$ corresponds to normal incidence.

\section{Comparison CWE/ROM}

As a conclusion to this parametric study, we compare the features of the CWE an ROM processes. Concerning the dependence on the laser peak intensity, we showed in Sec. IVE that CWE has an almost constant generation efficiency in a large intensity range above a few $10^{15}$ $\mathrm{W} / \mathrm{cm}^{-2} \mu \mathrm{m}^{2}$. Below this range, the efficiency abruptly drops down (see Sec.VI C 1). Roughly speaking, the generation efficiency of ROM follows a similar behavior for much higher intensities, where the efficiency reaches a saturation. A major difference is that the ROM efficiency grows with intensity in a broad range $\left(10^{18}-10^{20}\right.$ $\left.\mathrm{W} / \mathrm{cm}^{-2} \mu \mathrm{m}^{2}\right)$, whereas this dependence is always very weak for CWE.

The influence of the gradient scale length is quite different in the two mechanisms. Indeed, an optimal scale length exists for CWE, whereas the efficiency of ROM rises with $L$ for $L \lesssim \lambda / 5$. As the optimal length for CWE is extremely short (about $\lambda_{L} / 50$ ), this means for practical purposes, that short gradients tend to favor CWE (depending on the laser intensity), while long gradients favor ROM. Changing the gradient scale length can thus be a way to control the relative strengths of the two mechanisms [60].
In contrast, CWE and ROM have very similar dependences on the incidence angle, except around $\theta=0$. In particular, the optimal angle for both mechanisms is about $55^{\circ}$. This similarity is explained by the central role played, in both cases, by the component of the laser electric field along the plasma normal.

\section{H. Phase properties}

We now analyze the phase properties of ROM harmonics. To begin with, we stress that because all of the highest ROM harmonics are emitted precisely when the plasma surface reaches its maximal velocity, the attosecond pulses associated to these harmonics should be close to Fourier-Transform Limited (FTL) [83]. In other words, the so-called 'attosecond spectral phase' of ROM harmonics should be constant. By taking advantage of this fact and of the very broad spectra that can be generated, extremely short pulses, possibly down to the zeptosecond range, can potentially be produced by ROM [28]. In contrast, for multi-cycle laser pulses, there is no reason why the pulse train should be perfectly periodic, and the individual harmonics can therefore be chirped -i.e. the femtosecond spectral phase is not necessarily constant.

This issue has been studied in Ref. [62], where the properties (Carrier-envelop relative phase, emission time, chirp) of ROM attosecond pulses are observed to be almost independent of $I$, for $I \lambda_{L}^{2} \lesssim 10^{19} \mathrm{~W} / \mathrm{cm}^{2} \mu \mathrm{m}^{2}$. This means that for moderately relativistic intensities, the generated pulse trains are almost fully periodic, and individual harmonics are FTL. By contrast, Fig. 35 shows that, for very high laser intensities, the emission time of the attosecond pulses is intensity-dependent, which means that in this case harmonics have non-trivial phases, as already emphasized in [84]. Figure 35 also indicates that in the case of fixed ions, the sign of the femtosecond chirp is not the same in the CWE and ROM processes. Indeed, ROM harmonics are positively chirped, because the spacing between successive pulses in the train decreases in time.

This temporal variation of $\tau_{e}$ originates from the denting of the plasma surface, induced by the laser ponderomotive effect [85]. To illustrate this point, we performed a simulation with mobile ions, which result is plotted in Fig. 36. The grayscale map of the electron density clearly shows that the mean position of the critical surface is pushed inwards. As a consequence, the time needed for the laser field to reach the surface, and then the time needed for the attosecond pulses to reach the observer, both increase in time. The time at which a fixed observer detects an attosecond pulse is given by

$$
\tau_{e}(i)=t_{e}(i)+i T+2 \cos \theta x_{e}(i) / c,
$$

where $i$ is the number of the laser optical cycle, $t_{e}(i)$ is the delay after which an attosecond pulse is emitted within the $i^{t h}$ laser cycle (with respect to a fixed reference 


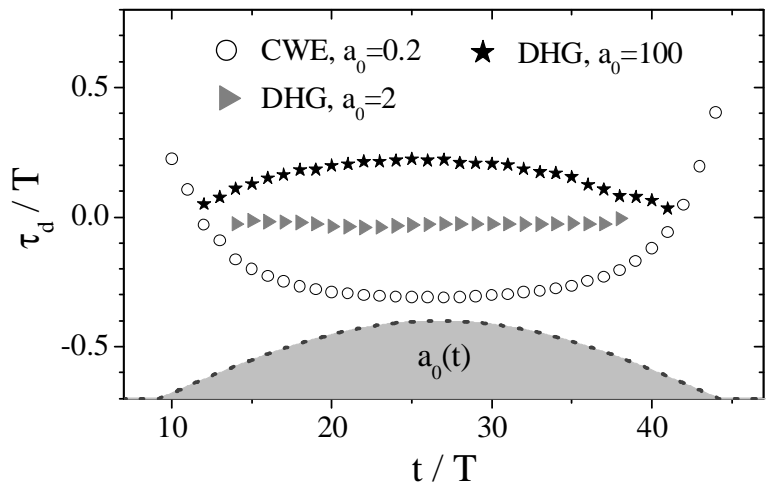

FIG. 35: Emission times of CWE and relativistic attosecond pulses. These curves show the time dependence of the emission time for three different laser peak intensities. The three curves have been shifted vertically to simplify their comparison, absolute values of $\tau_{e}$ are thus meaningless. In these CALDER simulations, $n_{e}=110 n_{c}$. In the CWE case, $L=\lambda_{L} / 100$, while in the relativistic cases, $L=\lambda_{L} / 30$ for the moderate intensity, and $L=\lambda_{L} / 10$ for the highest one. The gray area shows the amplitude temporal profile of the laser pulse.

phase of the local laser field), and $x_{e}(i)$ the position of the critical surface at $t=i T_{L}+t_{e}(i)$. To check if the phase is really due to the surface denting, we assume that $t_{e}$ is constant, i.e. that the emission of the attosecond pulse always occur at the same local phase of the laser field, whatever the laser intensity. With this assumption, we then calculate the position of the emission points $x_{e}(i)$ using Eq. (39) and the emission times $\tau_{e}(i)$ provided by the PIC simulation. These points are plotted in Fig. 36, and are precisely localized on the critical surface, which proves that the variations in time of $\tau_{e}$ are due to the motion of the plasma surface, and not to an intrinsic dependence of $t_{e}$ on $I$, as in the case of CWE. Thus, the measure of $\tau_{e}(i)$ provides a way to monitor the slow dynamics of the plasma surface.

The time dependence of $\tau_{e}$ affects the properties of the harmonic spectrum. The main effect in the case of Fig. 36 is a red Doppler shift of the harmonics. Indeed the frequency of harmonic $n$ is $\omega_{n} \approx n \omega_{L}\left(1-2 \cos \theta v_{d r i f t} / c\right)$, where $v_{\text {drift }}$ is the mean drift velocity of the plasma surface. In the present case we have $v_{d r i f t} \approx 0.03 c$ and hence $\omega_{n} \approx 0.96 n \omega_{L}$. Note that because $\Delta \omega_{n}=\omega_{n}-n \omega_{L} \propto$ $n v_{\text {drift }}$, we can use the harmonic shift to measure slow motions of the plasma surface with a high precision, by considering sufficiently high harmonic orders.

While the main effect of hole boring in Fig. 36 is to introduce a spectral shift, harmonic phases have also nonlinear components because the surface has a non-constant velocity. This leads to a spectral broadening of individual harmonics and to non-trivial spectral phases. At very high intensities such as the one considered in Fig.36, the harmonic spectral widths are thus expected to be significantly larger than the Fourier-transform limit. Similar effects can also be observed at much lower laser inten-

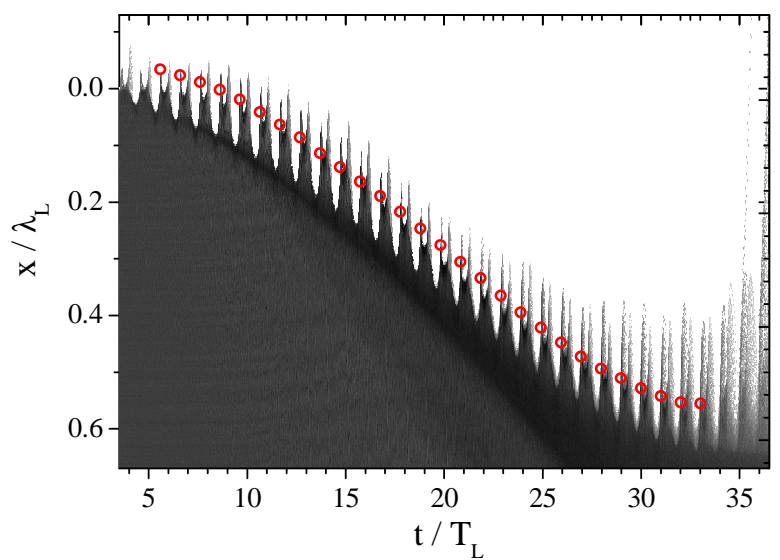

FIG. 36: Hole boring and femtosecond chirp. The grayscale map shows the electron density as a function of time and space. The circles are the calculated positions $\left(t_{e}, x_{e}\right)$ of the emission points. The numerical conditions are the same as in Fig. 35 for $a_{0}=100$, except that ions are now free to move.

sities, if the plasma has a lower density, or if a longer density gradient is present [85-87]. Note that when ions are fixed in simulations, as in the case of Fig. 35, the Doppler shift is suppressed, and harmonics are only spectrally broadened.

Finally, we already mentioned that the temporal effect described above has an equivalent in the spatial domain. The space-dependent laser intensity in the focal plane actually leads to a focusing of the harmonic beam, and increases it divergence. This effect was put forward in Ref. [64] to interpret the measured divergences of ROM harmonics.

\section{Conclusion}

The essential ingredient of the Relativistic Oscillating Mirror mechanism is the periodic Doppler effect induced on the reflected light, within each laser cycle, by the moving current distribution at the plasma-vacuum interface. We have shown here how to diagnose and assess the importance of this Doppler effect on the reflected light, for a given transverse current distribution $j_{t}(x, t)$ in the boosted frame. Such a Doppler effect indeed leads to a very specific shape of the $2 \mathrm{D}$ Fourier-transform $\hat{\hat{j}}_{t}(k, \omega)$ of $j_{t}(x, t)$.

In this work, this current distribution was simply obtained by PIC simulations. A predictive and quantitative analytical theory of the ROM mechanism will require determining a general expression of $j_{t}(x, t)$ in the relativistic interaction regime. To calculate the emitted harmonic spectrum and generation efficiency for ROM, this distribution is only needed around the times where the plasma move toward vacuum at relativistic velocities. Even then, given the complexity of the interaction, deriving such an expression is a very challenging task, and it is not clear 
yet that it will be possible at all in the most general case.

\section{EXPERIMENTAL EVIDENCE}

We now turn to the experimental evidence supporting the existence of the two HHG mechanisms described so far, CWE and ROM. We mostly focus our discussion on the experimental results obtained at CEA in the past five years. One essential conclusion from these recent studies is the fact that the harmonic signals associated to these two mechanisms can be distinguished unambiguously in experiments in the moderately relativistic regime, through a set of very different properties. These are the following:

(i) the spectral extension of the harmonic signal,

(ii) the dependence of this signal on laser intensity,

(iii) the spectral width of individual harmonics,

(iv) the divergence of the harmonic beam.

After a short description of our main experimental tools, we present several measurements of these different properties, and show how they can be used to discriminate between CWE and ROM.

\section{A. Experimental tools}

The first requirement for the experimental study of HHG on plasma mirror is to produce such a mirror, i.e. a dense plasma at the surface of a solid target, with a sharp interface with vacuum. In Sec. II, we showed that this can in principle be achieved simply by focusing an ultrashort and intense laser pulse on a solid target, which both generates the plasma during its leading edge, and generates the harmonics. In practice, this is greatly complicated by the light pedestal surrounding ultrashort laser pulses, which is an inherent feature resulting from the technology used in these lasers. This pedestal is much weaker than the main pulse -the ratio of their intensities being the temporal contrast- but generally much longer (up to nanoseconds). Without any special tool to reduce this pedestal, the temporal contrast is generally what imposes the maximum intensity $I_{M}$ at which a plasma mirror can be driven. Beyond $I_{M}$, the pedestal becomes intense enough to ionize the target well before the arrival of the main pulse. This pulse then interacts with an expanded plasma rather than with a plasma mirror.

The potentially dramatic effects of the pedestal on the reflected beam and harmonic signal are illustrated in Fig. 37. While the plasma behaves as a high-quality mirror at lower intensities, above $I_{M}$ the profile of the reflected laser beam is strongly distorted because of preplasma expansion. Further, Fig. 37 shows that HHG is suppressed for $I>I_{M}$, which proves that it is absolutely necessary to avoid any effect of the pedestal to produce high order harmonics at high intensity [88]. For this reason, the issue of the temporal contrast has kept hindering the experimental study of HHG in dense plasmas, until efficient tools to improve this contrast were developed a few years ago [89-93].

Two lasers, LUCA and UHI10, from the Saclay Laser Interaction Center (SLIC) Laserlab facility in CEA, France, have been used for our experimental investigations of HHG on plasma mirrors. Both lasers are based on the Chirped Pulse Amplification technique (CPA) [94], use Titanium-Sapphire as a laser medium, and deliver pulses at a central wavelength of $800 \mathrm{~nm}$.

LUCA is a $1 \mathrm{TW}$ laser chain, consisting of an oscillator followed by a regenerative amplifier and two multi-pass amplifiers, and delivering 40 fs pulses at a repetition rate of $20 \mathrm{~Hz}$. The laser beam was focused at the surface of bulk silica targets using a $200 \mathrm{~mm}$ focal length off-axis parabolic mirror, leading to a numerical aperture of $f / 5$ for the focused beam. The temporal intensity profile of this laser is shown in logarithmic scale in Fig. 38. The temporal contrast is typically $10^{-6}$ beyond 10 ps. Because of this rather poor contrast, only $10 \%$ of the 50 mJ provided by LUCA can be focused on the silica targets before they get altered by the pedestal (Fig. 37). This corresponds to a maximum intensity of $6 \times 10^{16} \mathrm{~W} / \mathrm{cm}^{2}$, much lower than the limit of a few $10^{18} \mathrm{~W} / \mathrm{cm}^{2}$ required to enter the relativistic interaction regime for an $800 \mathrm{~nm}$ laser field. As demonstrated by Fig. 37, this is however high enough to drive HHG by CWE.

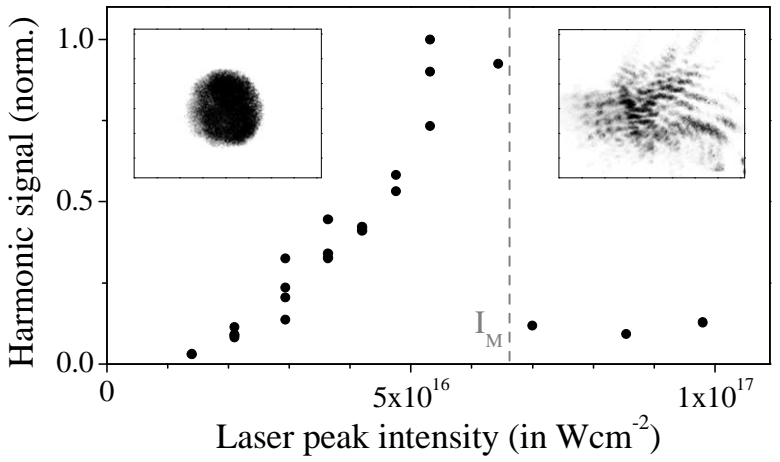

FIG. 37: Intensity dependence of the CWE harmonic signal induced by a femtosecond laser pulse of $\approx 10^{6}$ contrast on the ns time-scale (from the LUCA laser), hitting a silica target. This signal suddenly collapses when $I$ exceeds $I_{M} \approx 6 \cdot 10^{16}$ $\mathrm{W} / \mathrm{cm}^{2}$. The insets show the far-field intensity distribution of the laser beam after reflection on the plasma, for two different peak intensities, above and below $I_{M}$.

UHI10 has the same architecture as LUCA, but has one more multipass amplification stage. It delivers 60 fs pulses with an energy of up to $700 \mathrm{~mJ}$, leading to a peak power of about $10 \mathrm{TW}$. This laser was focused on either metallic or dielectric solid targets with off-axis parabolic mirrors of two different focal lengths, $200 \mathrm{~mm}$ or $500 \mathrm{~mm}$, corresponding to numerical apertures of respectively $f / 3.3$ and $f / 8.3$. With the $200 \mathrm{~mm}$ parabola, 


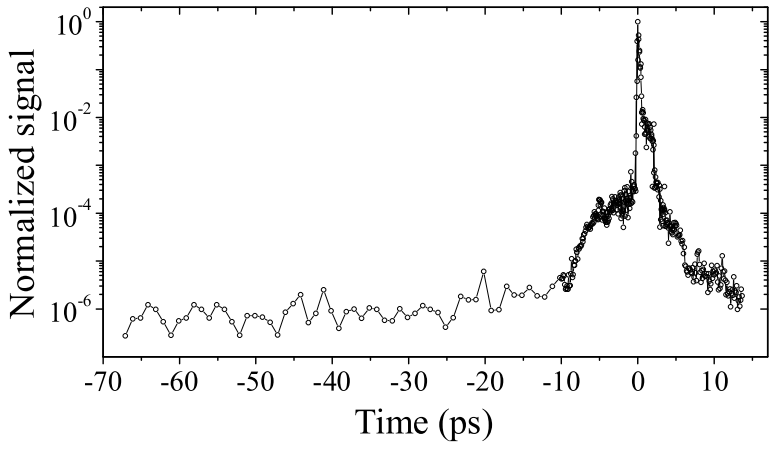

FIG. 38: Temporal profile of the LUCA laser over a 100 ps time range, measured with a high-dynamic third-order autocorrelator (SEQUOIA, Amplitude Technologies).

measurements of the laser focal spot size lead to an estimate of a maximum intensity close to $2.10^{19} \mathrm{~W} / \mathrm{cm}^{2}$.

The temporal contrast at the output of UHI0 is of the order of $10^{-6}$ beyond $10 \mathrm{ps}$, comparable to the LUCA laser. Driving HHG on plasma mirrors at the highest intensities provided by this laser thus first requires improving this contrast by several orders of magnitude. This is achieved using a double plasma mirror system as an ultrafast optical switch [93]. This system, permanently installed between the compressor and the experimental chamber of UHI10, is described in Ref. [95]. It improves the temporal contrast by about four orders of magnitude up to the very beginning of the laser pulse, leading to contrast of the order of $10^{10}$ on the $10 \mathrm{ps}$ time scale. The cost for this improvement is a loss of about $50 \%$ of the main laser pulse energy, thereby reducing the peak intensity on target to about $10^{19} \mathrm{~W} / \mathrm{cm}^{2}$ with the $200 \mathrm{~mm}$ parabola.

We now turn to the experimental study of HHG on plasma mirrors, performed with these two lasers.

\section{B. Spectral extension of the harmonic signal}

\section{Case of Coherent Wake Emission}

According to Sec. IV D, an essential feature of CWE is the fact that it results in a harmonic spectrum extending up to the maximum plasma frequency $\omega_{p}^{\max }$ of the plasma generated by the laser pulse on the initially-solid target. This frequency is given by $\omega_{p}^{\max }=\sqrt{n_{e}^{\max } e^{2} / m \epsilon_{0}}$, where $n_{e}^{\max }$ is the maximum electron density of this plasma.

As we have seen in Sec. II A, with ultrashort pulses (of high-enough temporal contrast) the expansion of the plasma is extremely weak during the laser pulse, and $n_{e}^{\max }$ is then given by $p n_{e}^{s}$, with $n_{e}^{s}$ the total electron density of the initial solid target, and $p$ the ionization fraction of the dense part of the target due to the interaction with the laser field. A rigorous calculation of $p$ is extremely complex, but for targets made of elements with low-enough $Z$ (such as silica $\mathrm{SiO}_{2}$, plastic $\mathrm{CH}$ or
Aluminum $A l$ ), one can safely assume $p \approx 1$ for laser intensities $I>10^{17} \mathrm{~W} / \mathrm{cm}^{2}$ (see Fig. 2(b) in Sec. II A).

A good way to identify CWE harmonics is thus to measure the harmonic spectral cut-offs obtained on targets with different electron densities. For laser intensities of up to a few $10^{18} \mathrm{~W} / \mathrm{cm}^{2}$ experiments performed with the UHI10 laser led to a harmonic frequency cut-off that varies as the expected target maximum plasma frequency (Fig. 39 and Tab. II). This is a clear indication of the dominant role of in this interaction regime.

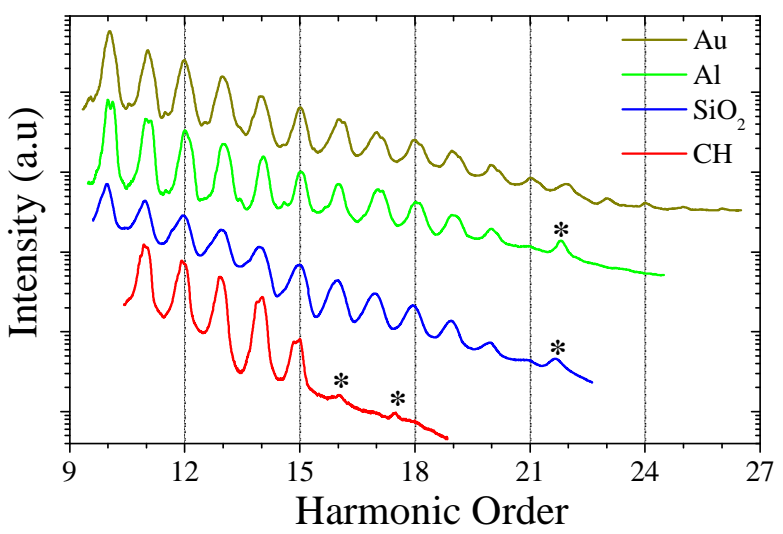

FIG. 39: (Color online) CWE harmonic spectra obtained at an intensity of $3 \times 10^{18} \mathrm{~W} / \mathrm{cm}^{2}$, on targets of different initial densities, with the UHI10 laser. The stars indicate incoherent plasma line emission. To make comparison easier, these different spectra have been arbitrarily shifted along the $y$ axis.

The case of gold, also presented in Fig. 39, is less conclusive. For a target with such a high $Z$ number $(Z=79), p$ is actually significantly lower than 1 even at these laser intensities, and it is difficult to determine its exact value, be it by numerical calculations or by an independent density measurement. However, assuming that CWE is the dominant generation mechanism, the harmonic spectrum cut-off of Fig. 39 at $n \approx 26$ for gold leads to $p \approx 25 \%$, which is realistic at the considered laser intensity.

\begin{tabular}{lcccc}
\hline \hline Materials & $\mathrm{CH}$ & $\mathrm{SiO}_{2}$ & $\mathrm{Al}$ & $\mathrm{Au}$ \\
$n_{e}^{s}$ in $\mathrm{cm}^{-3}$ & $4 \times 10^{23}$ & $7 \times 10^{23}$ & $8 \times 10^{23}$ & $5 \times 10^{24}$ \\
$\omega_{p}^{\text {max }}$ in $\omega_{L}$ & 15 & 20 & 21 & 53 \\
Max. order observed & 15 & $20-21$ & $20-21$ & $>26$ \\
\hline \hline
\end{tabular}

TABLE II: Maximum plasma density (totally ionized target with neglegible expansion), corresponding plasma frequency $\left(\omega_{p}^{\max }\right)$, and measured maximum harmonic order for plastic, silica, aluminum and gold targets.

Finally, we note that in the first experiments on high order harmonic generation on solid targets performed in $1981[18,19]$ with nanosecond laser pulses, Carman et al. already reported a frequency cutoff around $\omega_{p}^{\max }$. The major difference with the present results is that, in the 
nanosecond regime, the density profile in the interaction zone is largely the result of a competition between the thermal expansion of the plasma and the radiation pressure. The maximum plasma density is thus unknown $a$ priori, and this made the evidence for a cut-off at $\omega_{p}^{\max }$ highly uncertain.

\section{Case of Doppler harmonic generation}

As opposed to the case of CWE, there is no fixed limit to the maximum harmonic order that can be generated by the Doppler effect. In this case, the frequency cut-off depends mostly on the maximum velocity of the outgoing electron bursts at the plasma surface (see Sec. VE), which is expected to increase with laser intensity.

Observing this mechanism requires to increase the laser intensity typically beyond a few $10^{18} \mathrm{~W} / \mathrm{cm}^{2}$ for an 800 $\mathrm{nm}$ laser field -a limit that can actually shift toward lower intensities as the density gradient scale length increases [60]. Experimentally, we observed the sudden appearance of a large number of harmonics above the target maximum plasma frequency above approximately $5 \times 10^{18}$ $\mathrm{W} / \mathrm{cm}^{2}$ (Fig. 40). As these harmonics precisely appear when the laser intensity exceeds the threshold for the relativistic interaction regime, we attribute them to the Doppler effect. We will see that several other observations support this conclusion. We emphasize, however, that direct experimental evidence that these relativistic harmonics are indeed due to a Doppler effect remains to be obtained.
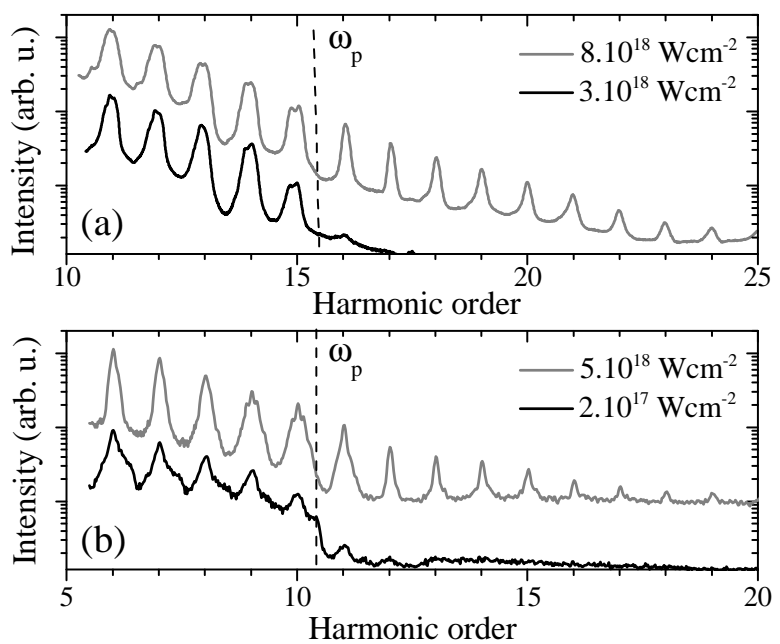

FIG. 40: Non-relativistic and relativistic angularly-integrated harmonic spectra measured with UHI10. (a) Experimental spectra obtained on a plastic target, for laser intensities of $3 \times 10^{18} \mathrm{~W} / \mathrm{cm}^{2}$ and $8 \times 10^{18} \mathrm{~W} / \mathrm{cm}^{2}$. (b) Calculated spectra obtained from $2 D$ CALDER simulations, in the case of a plasma of $110 n_{c}$ maximum density.

\section{Laser intensity dependence}

\section{Case of Coherent Wake Emission}

The second essential feature of CWE is its quasi-linear dependence on the laser intensity, all other things being equal. As we have seen in Sec. IV E 1, this unexpected property is clearly observed in numerical simulations, and is well understood by considering the intensity dependences of the different steps of the CWE process.

For laser intensities below a few $10^{18} \mathrm{~W} / \mathrm{cm}^{2}$, the harmonic signal intensity dependence measured experimentally is also quasi-linear (Fig. 41). This is further evidence of the dominance of the CWE mechanism in this intensity range. The measured exponent of the power law slightly varies in different experiments. This is probably due to the variation with the laser intensity of important physical parameters, such as the form and the length of the density gradient.

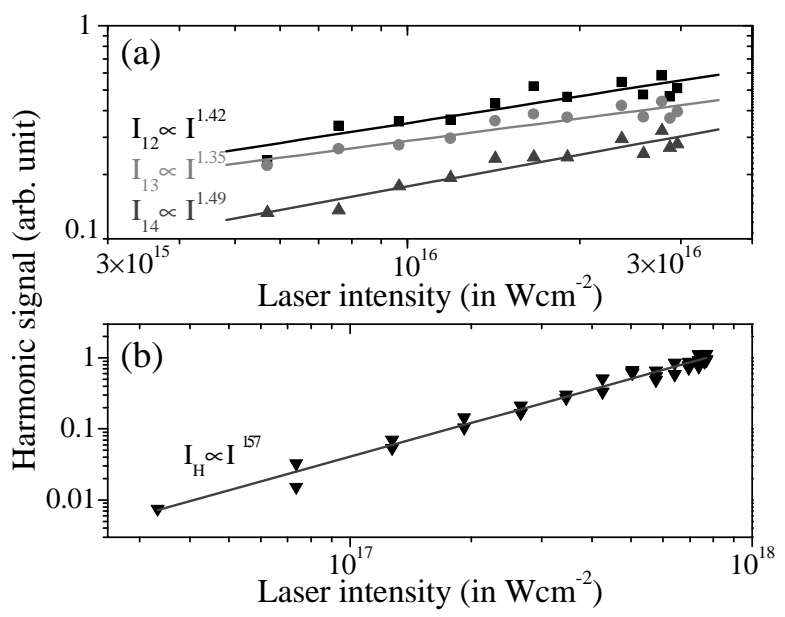

FIG. 41: (a) Intensity dependence of the harmonic signal for three different CWE harmonics, produced with the LUCA laser. The laser intensity was varied using an attenuator (combination of an half wave-plate and two polarizers). (b) Intensity dependence of the spatially and spectrallyintegrated harmonic signal, measured experimentally in the non-relativistic regime with the UHI10 laser. In these case the different points have been obtained by simply varying the laser polarization (from pure $p$ to pure $s$ ). The harmonic beam goes through a $200 \mathrm{~nm}$ thick Indium filter which mainly transmits orders 10 to 15 .

It is worth stressing that, because it does not rely on any highly non-linear coupling between the laser field and the plasma, CWE can be driven at laser intensities as low as a few $10^{15} \mathrm{~W} / \mathrm{cm}^{2}$, which initially came as a complete surprise. The laser intensity simply needs to be high enough to drive the Brunel absorption and accelerate electrons to velocities allowing them to cross within the density gradient (see Sec. IVE 1 ). Below $\approx 10^{15}$ $\mathrm{W} / \mathrm{cm}^{2}$, we observed experimentally that the CWE signal abruptly vanishes. 


\section{Case of Doppler harmonic generation}

As we have seen, observing relativistic harmonics requires increasing the laser intensity beyond the range covered by the measurements of Fig. 41. Figure 42 displays a contour map showing the evolution of the harmonic spectrum around order 15 with laser intensity, above and below the relativistic threshold. Since the target in this case consists in plastic, CWE does not contribute to the harmonic signal above order 15 . The higher order are thus attributed to the Doppler effect.

These two classes of harmonics have very different behavior when the laser intensity is reduced. The harmonic signal varies very smoothly below order 15 , as expected for CWE harmonics, while higher orders disappear almost as soon as the intensity is reduced, and almost all simultaneously. These harmonics thus have a highly nonlinear behavior near the relativistic threshold, which is characteristic of harmonics generated by the Doppler effect (see Sec. V G 1). These different behaviors thus provide a second mean to differentiate harmonics generated by CWE or ROM.

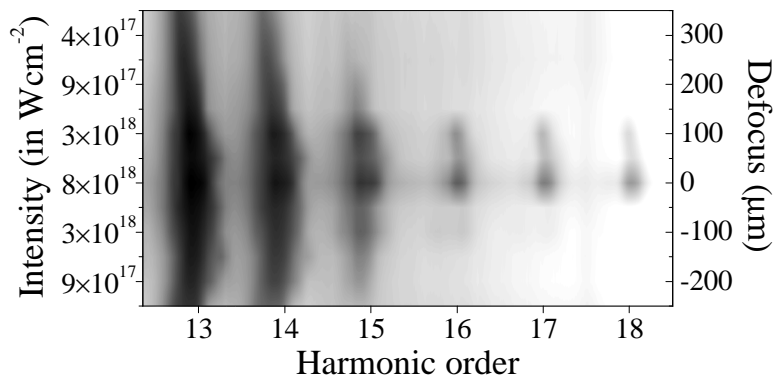

FIG. 42: Intensity dependence of the harmonic spectrum (orders 13 to 17$)$ generated on a plastic target $\left(\omega_{p}^{\max }=15 \omega_{L}\right)$, for $2 \times 10^{17} \mathrm{~W} / \mathrm{cm}^{2} \lesssim I \lesssim 8 \times 10^{18} \mathrm{~W} / \mathrm{cm}^{2}$. The signal is plotted in logarithmic scale. It was obtained using spectral profiles averaged over 5 laser shots, for 10 different laser intensities. The intensity was varied by changing the distance between the target surface and the best focus of the laser beam (right $y$ scale).

\section{Spectral width of individual harmonics}

Another striking difference between the two classes of harmonics associated to CWE and ROM appears on Fig. 40: the individual CWE harmonics have a significantly larger spectral width than the ROM ones. This is at first somewhat counterintuitive. Indeed, in the absence of a spectral phase of order 2 or higher, the spectral width of an individual harmonic is inversely proportional to the emission duration. Thus, considering the different intensity dependences of CWE an ROM, the ROM emission is expected to span a smaller fraction of the laser pulse than CWE, and should hence have larger spectral width than CWE, as opposed to what is observed experimentally.

The explanation for this apparent contradiction lies in the phase properties of the harmonics. As we have seen in Sec. IV F 3, the phase of CWE harmonics has a fairly strong dependence on the laser intensity. On the opposite, we observed in Sec. VH that the phase of ROM harmonics is almost independent of this intensity in the moderately relativistic interaction regime. Since the laser intensity varies in time in an actual experiment, according to the intensity envelop of the driving laser pulse, CWE harmonics have a large temporal phase -in other words, they are far from their FTL duration- while ROM harmonics do not. This temporal phase leads to a spectral broadening of the harmonics, which accounts for the difference observed between CWE and ROM harmonics.

This interpretation of the spectral width of CWE harmonics is corroborated by several experimental observations, which all support the theoretical and numerical analysis of Sec. IV F. Experimental evidence of the negative intrinsic chirp of CWE harmonics was first obtained in Ref. [62], in which this chirp, and the associated spectral broadening, were partially compensated by introducing a positive chirp on the driving laser pulse. In addition, a direct measurement of the intensity dependence of the emission time of CWE attosecond pulses using an interferometric technique agrees well with numerical results [96]. Finally, the analysis of Sec. IV F showed that the phase variations of CWE harmonics only depends on the laser pulse temporal intensity profile, but not on its peak intensity. This is confirmed experimentally by the fact that the spectral width of CWE harmonics is observed to be independent of the peak laser intensity (Fig. 43(a)).

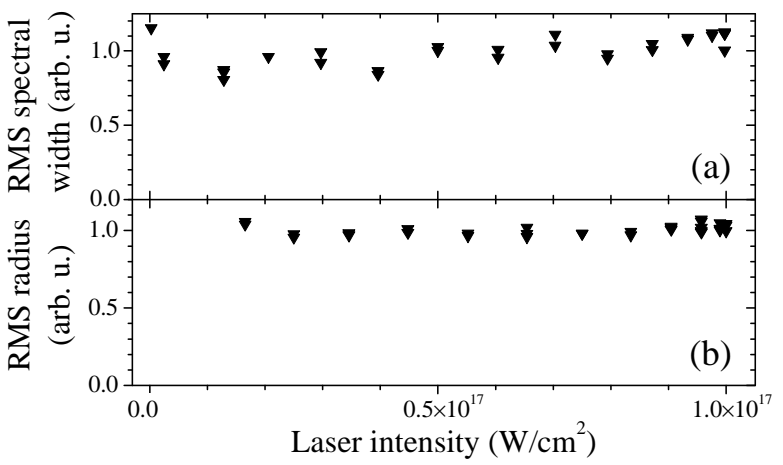

FIG. 43: Experimental measurements of the properties of CWE harmonics generated on silica, as a function of the peak laser intensity. (a) RMS spectral width of harmonic 14, (b) RMS divergence of the superposition of harmonics 10 to 15 , selected with a $100 \mathrm{~nm}$ thick In filter. 


\section{E. Divergence of the harmonic beam}

The intensity dependence of the harmonic phase, which has just been analyzed in the spectral domain, has analogous consequences in the spatial domain. Indeed, at the target surface, the laser intensity varies not only temporally, but also spatially. When the generation mechanism leads to a phase that depends on laser intensity -as in the case of CWE- the spatial variation of this intensity induces a spatial phase on the harmonic beam, and, for a given harmonic source size, this spatial phase results in an increase of the beam divergence.

The direct influence of this phase on the divergence of CWE harmonics has been proved experimentally in Ref. [62], using the same method as in the spectral domain. The intrinsic spatial phase was partly compensated by applying a spatial phase of opposite sign on the driving laser beam, obtained by simply moving the target slightly away from the best focus. Moreover, in Fig. 43(b), the divergence of the CWE harmonic beam, and hence the intrinsic spatial phase, is observed to be independent of the laser peak intensity, as predicted in Sec. IV F and observed in the spectral domain (Fig. 43(a)).

Due to these phase effects, and in analogy to the spectral domain, ROM harmonics are expected to have a weaker divergence than CWE harmonics, despite a smaller source size. This prediction is confirmed by $2 \mathrm{D}$ numerical simulations performed with the CALDER code (Fig. 44). This essential difference between the two classes of harmonics has recently been verified experimentally, on the ASTRA laser at the Rutherford Appleton Laboratory [64].

Our interpretation of this effect is also supported by the results of PIC simulations displayed in Fig. 45, which shows the intensity of the generated attosecond pulses as a function of the spatial coordinates $(x, y)$ in the vicinity of the source (i.e. within the Rayleigh length). It clearly demonstrates that ROM attosecond pulses are generated over a smaller radius than CWE pulses, due to the stronger intensity dependence of the ROM efficiency. It also shows that the CWE pulses are bent because of the intensity dependence of their emission time, while the ROM ones are not. This explains the smaller divergence of the ROM signal compared to the CWE one, despite a smaller source size.

Finally, in Fig. 44, we observe that below $\omega_{p}^{\max }$, CWE and ROM harmonics interfere, leading to a dark ellipse around each harmonic in the $(\omega, \theta)$ space. Understanding this interference pattern requires considering the phase properties of both mechanisms. In the case of CWE, we have shown that the dependence of the phase on the laser intensity induce both spatial and spectral non-trivial phases. If we assume that they are quadratic, we can write for a given harmonic $E^{C W E}(\omega, r) \propto e^{i\left(\omega t+\alpha_{\omega} \omega^{2}+\alpha_{\theta} \theta^{2}\right)}$. In contrast, in the weakly relativistic regime the phase of ROM harmonics is almost independent of $I$, and we have $E^{R O M}(\omega, \theta) \propto e^{i \omega\left(t-t_{0}\right)}$. The interference term in the

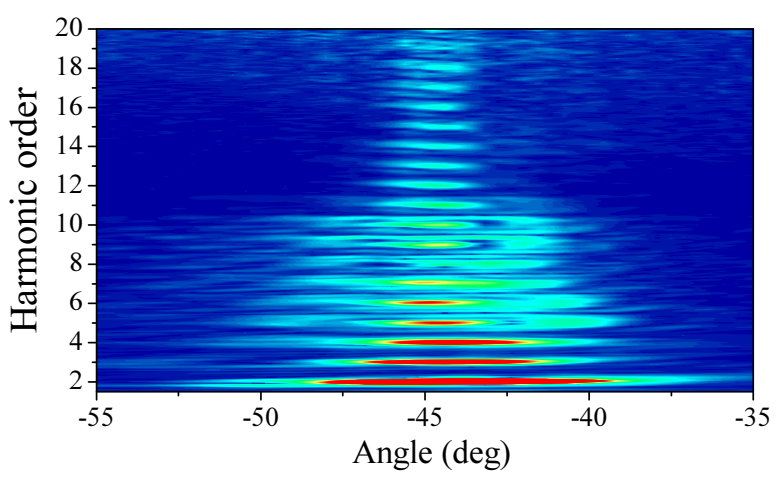

FIG. 44: (Color online) Angularly-resolved harmonic spectrum, in the moderately relativistic regime $\left(a_{0}=1.7\right)$, obtained from a $2 D$ CALDER simulation. This spectrum was corrected by a smooth function $T(\omega)$, to make it possible to observe simultaneously the angular width of all individual harmonics. An angle of $-45^{\circ}$ corresponds to the direction of specular reflexion, and the divergence of the incident Gaussian laser beam is $\pm 7.3^{\circ}$. In this simulation, the maximum plasma density is $n_{e}^{\max }=110 n_{c}$, harmonics beyond order 10 are therefore necessarily due to ROM.

spectrum $S(\omega, r)=\left|E^{C W E}(\omega, r)+E^{R O M}(\omega, r)\right|^{2}$ is thus proportional to $\cos \left(\omega t_{0}+\alpha_{\omega} \omega^{2}+\alpha_{\theta} \theta^{2}\right)$. This means that destructive interference occur on the ellipse of equation $\alpha_{\omega}\left(\omega+t_{0} / 2 \alpha_{\omega}\right)^{2}+\alpha_{\theta} \theta^{2}=\pi / 2+t_{0}^{2} / 4 \alpha_{\omega}^{2}$, in agreement with Fig. 44.

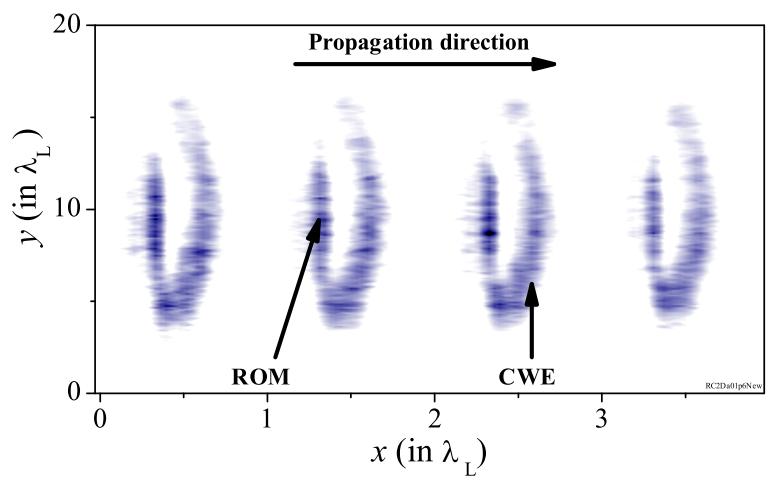

FIG. 45: (Color online) Intensity of the attosecond pulses resulting from the superposition of harmonics 7 to 15 , produced during the 4 central optical cycles of a laser pulse with $a_{0}^{\max }=1.7$, in a $2 D$ CALDER simulation where $n_{e}^{\max }=110 n_{c}$ and $L=\lambda_{L} / 50$.

\section{F. Summary and comparison to numerical simulations}

In summary, we have demonstrated in this section that it is possible to produce experimentally CWE and ROM harmonics, and that these two classes of harmonics can be easily distinguished experimentally, through the extension of the harmonic spectrum, the intensity depen- 
dence of the harmonic signal, the spectral width of individual harmonics as well as their divergence.

All these features are fairly well reproduced by PIC simulations, as illustrated by the simulated harmonic spectra displayed in Fig. 40(b) which exhibit, in particular, the two spectral ranges, on each side of $\omega_{p}^{\max }$, where each harmonic family dominates, as well as a clear difference in spectral width between these two families. The intensity dependence is also qualitatively reproduced. However, to totally eliminate the ROM signal, a stronger decrease in intensity is required in simulations than in experiments. This is most likely because simulations are performed in 2D only. As the harmonic signal is generated over a smaller fraction of the laser focal spot in ROM than in CWE, the relative weight of CWE and ROM signal in an angularly-integrated spectrum depends on the geometry of the interaction. Assuming $\rho<1$ is the ratio between the ROM and CWE source radii, the ratio of the ROM and CWE integrated signals indeed scales as $\rho$ in $2 \mathrm{D}$ geometry, while it scales as $\rho^{2}$ in $3 \mathrm{D}$ geometry. This can explain the different intensity dependences of the spectrum in $2 \mathrm{D}$ simulations and in experiments.

\section{CONCLUSION}

This paper provides a detailed analysis of the mechanisms that dominate laser high-order harmonic generation on plasma mirrors: these are Coherent Wake Emission, and the Relativistic oscillating Mirror process. The clear experimental evidence obtained to support the existence of these two mechanisms have also been summarized.

As already emphasized in this paper, some interesting theoretical problems remain to be solved on these two mechanisms. This includes establishing, if possible, a predictive and general theory of ROM, in particular in the ultra-relativistic regime, and a proper and accurate theoretical treatment of the last step of the CWE process, i.e. attosecond light pulse emission by plasma oscillations in an inhomogeneous plasma. However, from our point of view, these basic generation mechanisms are now fairly well understood, and the main remaining challenges on the topic of HHG from plasma mirrors lie on the experimental side. The most important problem to address is the temporal characterization of light reflected from plasma mirrors, with a temporal resolution going down to the attosecond range. A recent experiment has provided a first evidence of the existence of attosecond pulses produced by CWE [33], but this measurement only provides a rough estimate of their duration, averaged over the train. Going beyond and accessing experimentally the exact temporal structure of the attosecond pulse trains produced on plasma mirrors has two main interest.

Firstly, such measurements will provide a new and unique probe of the laser-plasma interaction. For instance, in CWE, the frequency content of each individual attosecond pulse in the train is determined by the instan- taneous maximum density of the plasma, while its chirp is influenced by the instantaneous gradient scale length. If this frequency content and this chirp could be measured for each attosecond pulses in a CWE train, this would provide the temporal evolution of the maximum plasma density and gradient scale length, with unprecedented temporal resolution and accuracy. These are some of the quantities that are still difficult to predict with numerical codes, but which have a crucial influence on the laser-plasma interaction. ROM can also provide a very valuable probe of the plasma. At relativistic intensities, a significant hole boring can be induced by the laser at the surface of the plasma. This can be diagnosed to some extent by measuring the resulting Doppler spectral shift induced on ROM harmonics. However, a full temporal characterization of the ROM attosecond pulse trains would provide a much more detailed diagnostics, and make it possible to reconstruct the complete temporal dynamic of this effect, simply by looking at the variation of the emission time of attosecond pulses during the laser pulse (Fig. 36). As a last example, a measurement of the timing of CWE and ROM attosecond pulses with respect to the laser field would tell us when the plasma surface reaches its peak velocity toward vacuum (ROM pulses), and when laser-accelerated electrons return to the plasma (CWE pulses). Such a measurement over the complete train would show how these two times vary with laser intensity, and thus provide new insight into the laser-plasma interaction dynamics. We note that complete measurements of attosecond fields are now possible using techniques such as described in Refs. [97, 98], and have already been achieved experimentally by several groups, for attosecond fields produced in gas targets.

Secondly, HHG from plasma mirrors is at present one of the best candidates to obtain an attosecond source of second generation. From this point of view, ROM is clearly far more promising than CWE, although it is also more demanding in terms of laser performances in particular laser peak intensity and temporal contrast. Indeed, as opposed to CWE, it has the potential to produce extremely broad spectra, associated to attosecond pulses that are close to Fourier-Transform Limited. For a given laser intensity, the ROM emission efficiency and spectral extension can be increased significantly by increasing the density gradient scale length at the plasma mirror surface (see Fig. 33), which can be achieved using a well-controlled pre-pulse. However, a longer gradient also implies a 'softer' plasma, and hence a stronger hole boring effect by the laser field. This hole boring will eventually increase the divergence and degrade the spatial quality of the harmonic beam. The best plasma-mirror based attosecond source will be a result of a compromise between these two trends.

Another essential and challenging experimental development is to go from trains of attosecond pulses to single pulses, as has been done about 10 years ago for HHG in gases. Several schemes have already been proposed to gate the ROM attosecond pulse emission [99-101]. These 
schemes should become experimentally accessible as laser and ultrafast optics technology improve.

\section{Acknowledgments}

C. Thaury acknowledges the financial support of the AXA Research Fund, and F. Quéré the financial support of the European Research Council (ERC) for the "Ultrahigh-Intensity Plasma Optics" project (grant $\mathrm{n}^{\circ}$ 240013) -which includes the work presented here on plasma mirrors. The 2D PIC calculation were performed using the computing resources of the "Grand Equipement National de Calcul Intensif" (GENCI), under project number x2010056057, and those of the "Centre de Calcul Recherche et Technologie" (CCRT). The authors also acknowledge the financial supports of the "Région Ile de
France" through the SESAME 2006 grant, of the French Agence Nationale pour la Recherche (ANR), through the project BLAN06-3-134072. The research leading to these results has received funding from the EC's Seventh Framework Programme "LASERLAB Europe II" (FP7/2007-2013) under grant agreement $\mathrm{n}^{\circ} 228334$.

Finally, the authors are extremely grateful to Guy Bonnaud and Erik Lefebvre for respectively providing the EUTERPE and CALDER codes, and for their assistance in the use of these codes. We are also greatly indebted to Rachel Nuter for her precious and efficient help in handling CALDER. Enriching scientific discussions with Jean-Paul Geindre, Patrick Audebert, Philippe Martin, Pascal Monot, Hervé George, Rolf Loch, Robin Marjoribanks and Matt Zepf were greatly appreciated. We thank Henri Vincenti for his careful and critical reading of the manuscript.
[1] T. Pfeifer, C. Spielmann, and G. Gerber, Rep. Prog. Phys. 69, 443 (2006).

[2] G. Farkas and C. Tóth, Phys. Lett. A 168, 447 (1992).

[3] F. Krausz and M. Ivanov, Rev. Mod. Phys. 81, 163 (2009).

[4] P. M. Paul, E. S. Toma, P. Breger, G. Mullot, F. Augé, P. Balcou, H. G. Muller, and P. Agostini, Science 292, 1689 (2001)

[5] M. Hentschel, R. Kienberger, C. Spielmann, G. A. Reider, N. Milosevic, T. Brabec, P. Corkum, U. Heinzmann, M. Drescher, and F. Krausz, Nature 414, 509 (2001).

[6] P. Agostini and L. F. Di Mauro, Rep. Prog. Phys. 67, 813 (2004).

[7] G. Sansone, E. Benedetti, F. Calegari, C. Vozzi, L. Avaldi, R. Flammini, L. Poletto, P. Villoresi, C. Altucci, R. Velotta, et al., Science 314, 443 (2006).

[8] E. Goulielmakis, M. Schultze, M. Hofstetter, V. S. Yakovlev, J. Gagnon, M. Uiberacker, A. L. Aquila, E. M. Gullikson, D. T. Attwood, R. Kienberger, et al., Science 320, 1614 (2008).

[9] M. Drescher, M. Hentschel, R. Kienberger, M. Uiberacker, V. Yakovlev, A. Scrinzi, T. Westerwalbesloh, U. Kleineberg, U. Heinzmann, and F. Krausz, Nature 419, 803 (2002).

[10] M. Uiberacker, T. Uphues, M. Schultze, A. J. Verhoef, V. Yakovlev, M. F. Kling, J. Rauschenberger, N. M. Kabachnik, H. Schröder, M. Lezius, et al., Nature 446, 627 (2007).

[11] A. L. Cavalieri, N. Müller, T. Uphues, V. S. Yakovlev, A. Baltuška, B. Horvath, B. Schmidt, L. Blümel, R. Holzwarth, S. Hendel, et al., Nature 449, 1029 (2007).

[12] S. Bahk, P. Rousseau, T. A. Planchon, V. Chvykov, G. Kalintchenko, A. Maksimchuk, G. A. Mourou, and V. Yanovsky, Opt. Lett. 29, 2837 (2004).

[13] W. Ackermann, G. Asova, V. Ayvazyan, A. Azima, N. Baboi, J. Bähr, V. Balandin, B. Beutner, A. Brandt, A. Bolzmann, et al., Nature Photon. 1, 336 (2007).

[14] S. V. Bulanov, T. Esirkepov, and T. Tajima, Phys. Rev. Lett. 91, 085001 (2003).
[15] M. Kando, Y. Fukuda, A. S. Pirozhkov, J. Ma, I. Daito, L.-M. Chen, T. Z. Esirkepov, K. Ogura, T. Homma, Y. Hayashi, et al., Phys. Rev. Lett. 99, 135001 (2007).

[16] T. Z. Esirkepov, S. V. Bulanov, M. Kando, A. S. Pirozhkov, and A. G. Zhidkov, Phys. Rev. Lett. 103, 025002 (2009).

[17] N. H. Burnett, H. A. Baldis, M. C. Richardson, and G. D. Enright, Appl. Phys. Lett. 31, 172 (1977).

[18] R. L. Carman, D. W. Forslund, and J. M. Kindel, Phys. Rev. Lett. 46, 29 (1981).

[19] R. L. Carman, R. F. Benjamin, and C. K. Rhodes, Phys. Rev. A 24, 2649 (1981).

[20] B. Bezzerides, R. D. Jones, and D. W. Forslund, Phys. Rev. Lett. 49, 202 (1982).

[21] C. Grebogi, V. K. Tripathi, and H.-H. Chen, Phys. Fluids 26, 1904 (1983).

[22] U. Teubner and P. Gibbon, Rev. Mod. Phys. 81, 445 (2009).

[23] D. von der Linde, T. Engers, G. Jenke, P. Agostini, G. Grillon, E. Nibbering, A. Mysyrowicz, and A. Antonetti, Phys. Rev. A 52, R25 (1995).

[24] S. Kohlweyer, G. D. Tsakiris, C.-G. Wahlström, C. Tillman, and I. Mercer, Opt. Commun. 117, 431 (1995).

[25] S. V. Bulanov, N. M. Naumova, and F. Pegoraro, Phys. Plasmas 1, 745 (1994).

[26] P. Gibbon, Phys. Rev. Lett. 76, 50 (1996).

[27] R. Lichters, J. Meyer-ter-Vehn, and A. Pukhov, Phys. Plasmas 3, 3425 (1996).

[28] S. Gordienko, A. Pukhov, O. Shorokhov, and T. Baeva, Phys. Rev. Lett. 93, 115002 (2004).

[29] T. Baeva, S. Gordienko, and A. Pukhov, Phys. Rev. E 74, 046404 (2006)

[30] U. Teubner, K. Eidmann, U. Wagner, U. Andiel, F. Pisani, G. D. Tsakiris, K. Witte, J. Meyer-ter Vehn, T. Schlegel, and F. E., Phys. Rev. Lett. 92, 185001 (2004).

[31] K. Eidmann, T. Kawachi, A. Marcinkevičius, R. Bartlome, G. D. Tsakiris, K. Witte, and U. Teubner, Phys. Rev. E 72, 036413 (2005).

[32] F. Quéré, C. Thaury, P. Monot, S. Dobosz, P. Martin, J.-P. Geindre, and P. Audebert, Phys. Rev. Lett. 96, 
125004 (2006).

[33] Y. Nomura, R. Hörlein, P. Tzallas, B. Dromey, S. Rykovanov, Z. Major, J. Osterhoff, S. Karsch, L. Veisz, M. Zepf, et al., Nature Phys. 5, 124 (2009).

[34] R. Ramis, R. Schmalz, and J. Meyer-Ter-Vehn, Comput. Phys. Commun. 49, 475 (1988).

[35] K. Eidmann, J. Meyer-ter Vehn, T. Schlegel, and S. Hüller, Phys. Rev. E 62, 1202 (2000).

[36] V. L. Ginzburg, The propagation of electromagnetic waves in plasmas (Oxford: Pergamon, 1970).

[37] D. W. Forslund, J. M. Kindel, K. Lee, E. L. Lindman, and R. L. Morse, Phys. Rev. A 11, 679 (1975).

[38] F. Brunel, Phys. Rev. Lett. 59, 52 (1987).

[39] P. Mulser, D. Bauer, and H. Ruhl, Phys. Rev. Lett. 101, 225002 (2008).

[40] A. Bourdier, Phys. Fluids 26, 1804 (1983).

[41] J. D. Jackson, Classical Electrodynamics (Wiley, 1998).

[42] A. S. Pirozhkov, S. V. Bulanov, T. Z. Esirkepov, M. Mori, A. Sagisaka, and H. Daido, Phys. Plasmas 13, 3107 (2006).

[43] J. M. Dawson, Rev. Mod. Phys. 55, 403 (1983).

[44] C. K. Birdsall and A. B. Langdon, Plasma Physics via Computer Simulation (McGraw-Hill, New York, 1985).

[45] G. Bonnaud and G. Reisse, Nucl. Fus. 5, 633 (1986).

[46] O. Coulaud, M. Dussère, P. Hénon, E. Lefebvre, and J. Roman, Parallel Comput. 29, 1175 (2003).

[47] D. V. Giovanielli and R. P. Godwin, Am. J. Phys. 43, 808 (1975).

[48] G. Bonnaud, P. Gibbon, J. Kindel, and E. Williams, Laser Part. Beams 9, 339 (1991).

[49] P. Chen, J. M. Dawson, R. W. Huff, and T. Katsouleas, Phys. Rev. Lett. 55, 1537 (1985).

[50] C. Thaury, Ph.D. thesis, Université Paris Sud 11 (2008).

[51] C. Thaury, F. Quéré, J.-P. Geindre, A. Levy, T. Ceccotti, P. Monot, M. Bougeard, F. Réau, P. D'Oliveira, P. Audebert, et al., Nature Phys. 3, 424 (2007).

[52] F. Quéré, C. Thaury, H. George, J. P. Geindre, E. Lefebvre, G. Bonnaud, S. Huller, P. Monot, and P. Martin, J. Mod. Opt. 55, 2711 (2008).

[53] Z.-M. Sheng, K. Mima, J. Zhang, and H. Sanuki, Phys. Rev. Lett. 94, 095003 (2005).

[54] Z.-M. Sheng, H.-C. Wu, K. Li, and J. Zhang, Phys. Rev. E 69, 025401 (2004).

[55] H. George, F. Quéré, C. Thaury, G. Bonnaud, and P. Martin, New J. Phys. 11, 113028 (13pp) (2009).

[56] R. W. Means, L. Muschietti, M. Q. Tran, and J. Vaclavik, Phys. Fluids 24, 2197 (1981).

[57] D. E. Hinkel-Lipsker, B. D. Fried, and G. J. Morales, Phys. Fluids B 4, 559 (1992).

[58] E. Ahedo and J. R. Sanmartin, Plasma Phys. Control. Fusion Fusion 29, 419 (1987).

[59] F. Quéré, C. Thaury, J.-P. Geindre, and P. Martin, Phys. Rev. Lett. 100, 089401 (2008).

[60] A. Tarasevitch, K. Lobov, C. Wünsche, and D. von der Linde, Phys. Rev. Lett. 98, 103902 (2007).

[61] B. Dromey, S. G. Rykovanov, D. Adams, R. Hörlein, Y. Nomura, D. C. Carroll, P. S. Foster, S. Kar, K. Markey, P. McKenna, et al., Phys. Rev. Lett. 102, 225002 (2009).

[62] F. Quéré, C. Thaury, J.-P. Geindre, G. Bonnaud, P. Monot, and P. Martin, Phys. Rev. Lett. 100, 095004 (2008).

[63] C. Thaury, F. Quéré, H. George, J.-P. Geindre, , P. Monot, and P. Martin, Eur. Phys. J. Special Top- ics 175, 43 (2009).

[64] B. Dromey, D. Adams, R. Hörlein, Y. Nomura, S. G. Rykovanov, D. C. Carroll, P. S. Foster, S. Kar, K. Markey, P. McKenna, et al., Nature Phys. 5, 146 (2009).

[65] D. A. Gurnett and R. R. Anderson, Science 194, 1159 (1976).

[66] T. S. Bastian, A. O. Benz, and D. E. Gary, Annu. Rev. Astron. Astrophys. 36, 131 (1998).

[67] P. A. Norreys, M. Zepf, S. Moustaizis, A. P. Fews, J. Zhang, P. Lee, M. Bakarezos, C. N. Danson, A. Dyson, P. Gibbon, et al., Phys. Rev. Lett. 76, 1832 (1996).

[68] D. M. Chambers, P. A. Norreys, A. E. Dangor, R. S. Marjoribanks, S. Moustaizis, D. Neely, S. G. Preston, J. S. Wark, I. Watts, and M. Zepf, Opt. Commun. 148, 289 (1998).

[69] M. Zepf, G. D. Tsakiris, G. Pretzler, I. Watts, D. M. Chambers, P. A. Norreys, U. Andiel, A. E. Dangor, K. Eidmann, C. Gahn, et al., Phys. Rev. E 58, R5253 (1998).

[70] I. Watts, M. Zepf, E. L. Clark, M. Tatarakis, K. Krushelnick, A. E. Dangor, R. M. Allott, R. J. Clarke, D. Neely, and P. A. Norreys, Phys. Rev. Lett. 88, 155001 (2002).

[71] J. Zhang, M. Zepf, P. A. Norreys, A. E. Dangor, M. Bakarezos, C. N. Danson, A. Dyson, A. P. Fews, P. Gibbon, M. H. Key, et al., Phys. Rev. A 54, 1597 (1996).

[72] U. Teubner, G. Pretzler, T. Schlegel, K. Eidmann, E. Förster, and K. Witte, Phys. Rev. A 67, 013816 (2003).

[73] B. Dromey, M. Zepf, A. Gopal, K. Lancaster, M. S. Wei, K. Krushelnick, M. Tatarakis, N. Vakakis, S. Moustaizis, R. Kodama, et al., Nature Phys. 2, 456 (2006).

[74] B. Dromey, S. Kar, C. Bellei, D. C. Carroll, R. J. Clarke, J. S. Green, S. Kneip, K. Markey, S. R. Nagel, P. T. Simpson, et al., Phys. Rev. Lett. 99, 085001 (2007).

[75] L. Plaja, L. Roso, K. Rzazewski, and M. Lewenstein, JOSA B 15, 1904 (1998).

[76] D. von der Linde and K. Rzàzewski, Appl. Phys. B 63, 499 (1996).

[77] A. Einstein, Ann. Phys. 17, 891 (1905).

[78] A. Gjurchinovski, Eur. J.Phys. 26, 643 (2005).

[79] D. An der Brügge and A. Pukhov, Phys. Plasmas 17, 033110 (2010).

[80] A. Pukhov and D. An der Brügge, Eur. Phys. J. D 58, 139 (2010).

[81] T. J. M. Boyd and R. Ondarza-Rovira, Phys. Rev. Lett. 100, 199502 (2008).

[82] T. J. M. Boyd and R. Ondarza-Rovira, Eur. Phys. J. D 58, 137 (2010).

[83] G. D. Tsakiris, K. Eidmann, J. Meyer-ter-Vehn, and F. Krausz, New J. of Phys. 8, 19 (2006).

[84] D. An der Brügge and A. Pukhov, Phys. Plasmas 14, 093104 (2007).

[85] S. C. Wilks, W. L. Kruer, M. Tabak, and A. B. Langdon, Phys. Rev. Lett. 69, 1383 (1992).

[86] R. Kodama, K. Takahashi, K. A. Tanaka, M. Tsukamoto, H. Hashimoto, Y. Kato, and K. Mima, Phys. Rev. Lett. 77, 4906 (1996).

[87] J. Fuchs, J. C. Adam, F. Amiranoff, S. D. Baton, P. Gallant, L. Gremillet, A. Héron, J. C. Kieffer, G. Laval, G. Malka, et al., Phys. Rev. Lett. 80, 2326 (1998). 
[88] P. Monot, G. Doumy, S. Dobosz, M. Perdrix, P. D'Oliveira, F. Quéré, F. Réau, P. Martin, P.Audebert, J. C. Gauthier, et al., Opt. Lett. 29, 893 (2004).

[89] D. Homoelle, A. L. Gaeta, V. Yanovsky, and G. Mourou, Opt. Lett. 27, 1646 (2002).

[90] R. Butkus, R. Danielius, A. Dubietis, A. Piskarskas, and A. Stabinis, Appl. Phys. B 79, 693 (2004).

[91] M. P. Kalashnikov, E. Risse, H. Schönnagel, and W. Sandner, Opt. Lett. 30, 923 (2005).

[92] A. Jullien, O. Albert, F. Burgy, G. Hamoniaux, J. Rousseau, J. Chambaret, F. Augé-Rochereau, G. Chériaux, J. Etchepare, N. Minkovski, et al., Opt. Lett. 30, 920 (2005).

[93] G. Doumy, F. Quéré, O. Gobert, M. Perdrix, P. Martin, P. Audebert, J. C. Gauthier, J.-P. Geindre, and T. Wittmann, Phys. Rev. E 69, 026402 (2004).

[94] D. Strickland and G. Mourou, Opt. Commun. 55, 447
(1985).

[95] A. Lévy, T. Ceccotti, P. D’Oliveira, F. Réau, M. Perdrix, F. Quéré, P. Monot, M. Bougeard, H. Lagadec, P. Martin, et al., Opt. Lett. 32, 310 (2007).

[96] C. Thaury, H. George, F. Quéré, R. Loch, J.-P. Geindre, P. Monot, and P. Martin, Nature Phys. 4, 631 (2008).

[97] Y. Mairesse and F. Quéré, Phys. Rev. A 71, 011401 (2005).

[98] F. Quéré, Y. Mairesse, and J. Itatani, J. Mod. Opt. 52, 339 (2005).

[99] N. M. Naumova, J. A. Nees, I. V. Sokolov, B. Hou, and G. A. Mourou, Phys. Rev. Lett. 92, 063902 (2004).

[100] T. Baeva, S. Gordienko, and A. Pukhov, Phys. Rev. E 74, 065401 (2006).

[101] S. G. Rykovanov, M. Geissler, J. Meyer-ter-Vehn, and G. D. Tsakiris, New J. Phys. 10, 025025 (2008). 